UNIVERSITE DU QUEBEC

MEMOIRE

PRESENTE A

L'UNIVERSITE DU QUEBEC EN ABITIBI-TEMISCAMINGUE

MAITRISE EN GESTION DES P.M.0.

PAR

GILLES FISET

IMPACT DU FINANCEMENT ACCREDITIF SUR L'ORGANISATION INTERNE ET LA STRATEGIE

LE CAS D'UNE JUNIOR EN EXPLORATION MINIERE

DECEMBRE 1987

Ce mémoire a èté réal isé à I'U.Q.A.T. dans le cadre du programme de Maîtrise en gestion des P.M.O. de I'U.Q.A.C. extensionné à I'U.Q.A.T. 


\section{Bibliothèque}

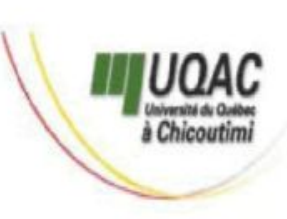

\section{Mise en garde/Advice}

Afin de rendre accessible au plus Motivated by a desire to make the grand nombre le résultat des results of its graduate students' travaux de recherche menés par ses research accessible to all, and in étudiants gradués et dans l'esprit des accordance with the rules règles qui régissent le dépôt et la governing the acceptation and diffusion des mémoires et thèses diffusion of dissertations and produits dans cette Institution, theses in this Institution, the I'Université du Québec à Université du Québec à Chicoutimi (UQAC) est fière de Chicoutimi (UQAC) is proud to rendre accessible une version make a complete version of this complète et gratuite de cette œuvre. work available at no cost to the reader.

L'auteur conserve néanmoins la The author retains ownership of the propriété du droit d'auteur qui copyright of this dissertation or protège ce mémoire ou cette thèse. thesis. Neither the dissertation or Ni le mémoire ou la thèse ni des thesis, nor substantial extracts from extraits substantiels de ceux-ci ne it, may be printed or otherwise peuvent être imprimés ou autrement reproduced without the author's reproduits sans son autorisation. permission. 


\section{REMERCIEMENTS}

J'aimerais exprimer mes remerciements aux personnes qui ont contribuè à la réalisation de cette recherche.

- Monsieur Yvan Charbonneau, en tant que directeur du programme de maitrise en gestion des petites et moyennes organisations, a permis cette recherche en acceptant ma proposition d'intégration de l'action et de la recherche. En tant que directeur de thèse, il m'a assuré de ses conseils et de sa disponibilité. Je puis dire que sa contribution me permettra d'évaluer différemment toute activité de recherche. En fait, il a su me démystifier cette activité humaine.

- Le personnel de la firme de secrétariat Alpha-Oméga pour l'application fournie lors de la dactylographie de cette recherche.

- Mes confrères, mes consoeurs et les professeurs du programme de maîtrise en gestion des petites et moyennes organisations pour leurs conseils et leurs échanges.

- Les membres de ma famille pour leur support moral tout au cours de cette démarche. 
REMERCIEMENTS

$\begin{array}{ll}\text { TABLE DES MATIERES } & \text { i }\end{array}$

LISTE DES TABLEAUX $\quad x i$

LISTE DES FIGURES $\quad x i i$

LISTE DES ANNEXES $\quad$ xi i

RESUME $\quad$ xiv

INTRODUCTION GENERALE XV

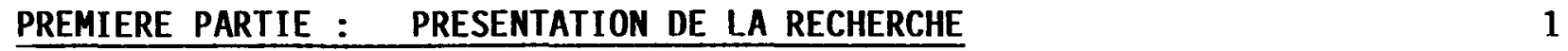

INTRODUCTION

$\begin{array}{lll}1.1 & \text { Le contexte de la recherche } & 3\end{array}$

1.1.1 Le chercheur-acteur

1.1.2 L'expérience d'entrepreneurship

1.1.3 Programme de maitrise en P.M.0.

1.1.4 Mémoire de recherche-action

1.1.5 Travaux de base

1.1.6 Choix du sujet

1.2 L'entreprise $\quad 5$

$\begin{array}{lll}1.3 & \text { La problématique } & 6\end{array}$

1.3.1 Evolution de la problématique 8

$\begin{array}{lll}1.4 & \text { Type de recherche } & 8\end{array}$

1.5 Objectifs de 1a recherche 9

$\begin{array}{lll}1.6 & \text { Méthodologie } & 10\end{array}$

CONCLUSION 
$\begin{array}{ll}\text { INTRODUCTION } & 14\end{array}$

$\begin{array}{lll}2.1 & \text { L'environnement } & 15\end{array}$

2.1.1 Les actions accréditives 15

2.1.1.1 description 15

2.1.1.? base dil système accréditif 16

2.1.1.3 fonctionnement 16

2.1.1.4 traitement fiscal 18

2.1.1.5 avantages des actions accréditives $\quad 19$

2.1.2 Les commandites et autres 21

2.1.2.1 diversité d'application ??

2.1.3 Le marché boursier 2.4

2.1.3.1 définition 24

2.1 .3 .2 bourses 24

2.1.3.3 marché primaire 24

2.1.3.4 marché secondaire 25

2.3.3.5 maisons de courtage 2.5

2.1.4 Marchē des métaux 28

2.1.4.1 l'industrie canadienne et mondiale $\quad 28$

2.1.4.2 T'activité économi que 28

2.1.4.3 les métaux précieux 28

2.1.4.4 effets sur 1'exploration 29

2.1.4.5 perspective 29

2.1.5 L'organisation de l'industrie de l'exploration 29

2.1.5.1 l'industrie 29

2.1.5.2 compagnies d'exploration 30

2.1.5.3 catégorie de juniors 30

2.1.5.4 catégorie de majeures 33

?.1.6 Les acteurs 33

2.1.6.1 fournisseurs 33

2.1.6.2 les investisseurs 34

2.1.6.3 les clients 34 
$\begin{array}{lll}2.1 .7 & \text { Politique } & 37\end{array}$

2.1.7.1 pēriode 1977-1987 37

2.1.7.2 état de la situation 40

2.1.8 Légat 44

2.1.8.1 bourse de Montréal 44

2.1.8.2 exigences de la Bourse de Montréal 44

2.1.8.3 actions de fondation et la

réglementation de la Commission

des valeurs mobilières du Québec 46

2.1.9 Impact économique 48

2.1.9.1 dépenses d'exploration FEC. 48

?.1.9.2 autres dépenses 50

2.1.9.3 découvertes 51

?.1.10 Système de financement 54

2.1.10.1 placement privé 54

2.1.10.2 le prospectus lors du placement public 55

2.1.10.3 le prospectus provisoire 56

2.1.11 Technologie 56

2.1 .11 .1 constat 56

2.1.11.2 firmes de consultation 57

2.1.11.3 évolution de la technologie 57

2.1.11.4 tendances 58

2.1.12 Physique 58

2.1.12.1 terrains miniers 58

2.1.12.2 T'Abitibi 58

2.1.12.3 la lncalisation 59

2.1.13 Concurrence 59

2.1.13.1 formes de concurrence 59

2.1.13.2 compétition indirecte 59

2.1.13.3 compétition directe 60

2.1.13.4 la compétition pour les terrains 61 
2.2 Le système étudiē

2.2.1 Organigramme

2.2.1.1 l'assemblée des actionnaires

2.2.1.2 le Conseil d'administration

62

2.2.1.3 officiers

$6 ?$.

2.2.1.4 direction

2.2.2 Rôle du Conseil d'administration

2.2.3 Description des tâches

?.2.3.1 directeur génēral

2.2.3.2 responsable de 1 'exploration

65

2.2 .3 .3 contrôleur financier

66

2.2 .3 .4 politiques opérationnelles

66

2.2 .3 .5 règl ements

2.2.3.6 aménagement

66

66

2.3 Le processus de transformation

2.3.1 Description du processus

2.3.? Explication du processus

2.4 Les sous-systèmes de 1 'entreprise

2.7.1 Ressources humaines

2.4.1.1 composition

2.4.1.2 expérience $\quad 73$

2.4.1.3 les consultants 73

2.4.1.4 propriētaires - Dirigeants 75

$\begin{array}{lll}2.4 .2 & \text { Finances } & 75\end{array}$

2.4.2.1 états financiers $\quad 75$

$\begin{array}{ll}2.4 .2 .2 \text { dilution } & 76\end{array}$

?.4.2.3 ententes 77

2.4.2.4 avenir financier 78 
$\begin{array}{lll}2.4 .3 & \text { Technologie } & 79\end{array}$

2.4.3.1 gestion de 1 'entreprise $\quad 79$

$\begin{array}{lll}2.4 .3 .2 & \text { gestion de T'exploration } & 79\end{array}$

2.4.4 Le marketing 80

2.4.4.1 prérequis pour une junior 80

2.4.4.2 le communiqué de presse comme outil de promotion 80

2.4.4.3 les propriétés comme base de promotion 81

2.4.4.4 le produit 8 ?

2.4.4.5 différenciations du produit 84

2.4.4.6 réseau de distribution 84

2.4.4.7 la différenciation des analystes 84

2.4.4.8 la concurrence 85

2.4.4.9 publicité et advertising 87

2.4.4.10 1a complexité de 1'industrie 87

2.4.4.11 le MIX marketing et la décision 88

2.4.4.12 utilité du marketing 89

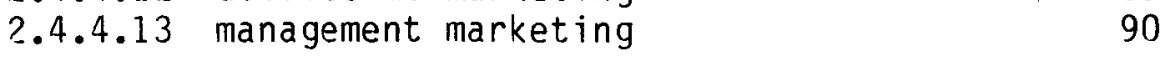

$\begin{array}{ll}2.4 .5 & \text { Les actifs }\end{array}$

2.4.5.1 les propriētés minières 90

2.4.5.1.1 titres et propriētés 90

2.4.5.1.2 obligations financières 9 ?

2.4.5.1.3 règles de base dans le choix 93 des propriétés

2.4.5.2 propriétés La Sarre, Brouillan,

Dal quier et Bassignac

$\begin{array}{lll}\text { 2.4.5.3 propriētē Barraute } & 94\end{array}$

2.4.5.4 propriēté Normétal 94

2.4.5.4.1 gîte Normetmar 95

$\begin{array}{ll}2.4 .5 .4 .2 & \text { historique du gite } \\ & \text { Normetmar }\end{array}$

2.4.5.4.3 études sur le gisement 96

2.4.5.4.4 méthode de minage du
scénario 3 
2.4.5.4.5 évaluation financière du scēnario 3

2.4.5.4.6 recommandations de 1 'expert-conseil

2.4.5.5 résidu minier de Normétal

104

2.4.5.5.1 étude quantitative sur le résidu minier

2.4.5.5.2 résultats de 1 'étude

?.4.5.5.3 étude de faisabilité

2.4.5.5.4 1 'entente avec Doelcam inc.

2.4.5.5.5 décision de production

2.4.5.6 liquidités et placements

2.4.5.7 qualité des actifs

2.4.5.7.1 effets des cycles sur les actifs miniers

2.4.5.7.2 santé financière
106

107

107

104

104

105

105

105

108

108

108

109

109

110

\subsection{La zone problématique}

2.6.1 Interrelation des problèmes

2.6.2 Problèmes d'acquisition

2.6.3 Problèmes de financement 
2.6.4 Problèmes de performance des ressources

112

2.6.5 Choix du problème et justification

113

CONCLUSION

114

\begin{tabular}{ll} 
TROISIEME PARTIE : ELABORATION D'UNE SOLUTION & 115 \\
\hline
\end{tabular}

$\begin{array}{lr}\text { INTRODUCTION } & 116\end{array}$

$\begin{array}{lll}3.1 & \text { La perspective } & 117\end{array}$

3.1.1 Système et sous-systèmes

3.2 Choix d'un système d'activitēs pertinent au problème 118

3.2.1 Examen de la littérature 118

3.2.2 Particularité de l'exploration minière 123

3.2.3 Définition d'un modèle 124

3.3 Modèle conceptuel

3.3.1 Explication du modèle

3.4 Comparaison avec la réalité

Développements

3.4 .2

Fonds de roulement

3.4 .3

Performance boursière

131

3.4 .4

Ressources humaines

131

3.4 .5

Résultats de 1 'exploration

131

3.4 .6

Programme d'acquisition 
3.5 Points forts et faibles

3.5.1 Situation financière

3.5.2 Propriétés

3.5.3 Ressources humaines

133

3.5.4 Financement

3.6 Recommandations

3.6.1 Stratégie à court terme

3.6.1.1 actifs miniers et financiers

133

3.6.1.2 ressources humaines

3.6.2 Réévaluation de la stratégie

4.1 Objectif de création d'entreprise 
4.3 Objectif de réflexion conceptuelle

4.3.1 Réflexion sur les concepts administratifs

143

4.3.2 Connaissances générales

4.4 Dualité des rôles de chercheur-acteur

144

CONCLUSION

145

CONCLUSION GENERALE 


\section{LISTE DES TABLEAUX}

page

Tableau I Application du système accréditif

Tableau II Maisons de courtage

Tableau III Types de compagnies minières

Tableau IV Tableau des investisseurs

Tableau $V$ Retombées économiques des dépenses d'exploration (en millier de \$) emplois et valeur ajoutée

Tableau VI Retombēes économiques des dépenses d'exploration

Tableau VII Courbe du produit 


\section{LISTE DES FIGURES}

Page

$\begin{array}{lll}\text { Figure I L'environnement } & 17\end{array}$

Figure II Organigramme Normétal inc. $\quad 64$

Figure III Processus de transformation des activitēs 68 de Normétal inc.

Figure IV Section longitudinale du projet Normetmar scénario $3 \quad 100$

Figure V Approches stratēgiques 119

$\begin{array}{ll}\text { Figure VI Modèle conceptuel de la planification } & 125\end{array}$ 


\section{LISTE DES ANNEXES}

Page

Annexe I Liste des maisons de courtage

Annexe II Carte des ceintures minières 


\section{RESUME}

Le présent travail de recherche intitulé: "Impact du financement accrēditif sur l'organisation interne et la stratégie. Le cas d'une junior en exploration minière" est 1 'aboutissement d'une démarche de recherche-action effectuée par Gilles fiset dans le cadre de la maitrise en gestion des petites et moyennes organisations à l'Université du Québec en Abitibi-Témiscamingue auprès d'Exploration minière Normétal inc. Il s'agit d'un cas de création d'entreprise, de même que de la production d'un travail de recherche à partir du vécu de cette création d'entreprise.

L'approche méthodologique est celle du diagnostic, lequel diagnostic est posé lors de la phase de démarrage de l'entreprise. Elle est enrichie d'éléments descriptifs et d'étapes d'analyse empruntées à la méthodologie des systèmes souples de Peter Chekcland. La phase de création de l'entreprise s'étend de septembre 1985 à décembre 1986.

Le mémoire prend la forme d'un diagnostic avec une description élaborée de l'organisation et de sa situation problématique. Suit une analyse de la réalité qui permet de dégager des points forts et faibles sur l'organisation interne de l'entreprise étudiée de manière à énoncer une certain nombre de recommandations. Les recommandations visent principalement la gestion de la stratégie de l'entreprise.

La validation de l'intervention se fait par l'action, la création de 1 'entreprise. En fait, le marché boursier valide la création de l'entreprise. Les objectifs cognitifs sont traités dans l'analyse méthodologique. L'analyse permet une évaluation des connaissances que le diagnostic-intervention permet d'obtenir pour l'acteur et 1 'entreprise, Exploration minière Normétal inc. La critique méthodologique précise aussi en quoi les connaissances administratives et conceptuelles furent acquises lors de la recherche. Elle se termine par une évaluation de la faisabilité de la recherche-action lorsqu'il y a dualité des rôles. 


\section{INTRODUCTION GENERALE}

L'analyse est le point de départ de toute décision. Elle peut être superficielle ou complète. Le diagnostic est une forme structurée d'analyse. L'analyse de l'environnement et de l'entreprise est primordiale pour permettre à cette dernière de mieux gērer son futur et sa survie; c'est ainsi que la planification stratégique existe et sert les entreprises.

Depuis quelques années, un certain nombre de facteurs ont favorisé 1 'émergence de compagnies d'exploration minière. Ce phēnomène est relativement nouveau au Quëbec; il est aussi mal connu. Peu de données, de connaissances et d'écrits existent sur ce secteur d'activité.

Le système de financement a des conséquences sur la pérennité des entreprises. Elles doivent assurer leur développement à partir de certains ëléments gẻnéralement reconnus comme essentiels à leur qualité: les propriétés minières, l'expertise technique et la promotion.

Dans cette recherche, nous déterminerons comment ces trois éléments se manifestent dans les fonctions de l'entreprise: finance, personne1, marketing et production. En particulier, nous étudierons comment ces trois élēments se manifestent dans notre entreprise diagnostiquēe, Exploration minière Normētal inc.

La première partie du mémoire présente l'entreprise étudiée ainsi que la problématique globale. Cette partie situe aussi le lecteur sur le type de recherche effectuée et les procēdés utilisés pour la réaliser, à savoir: la méthodol ogie.

La deuxième partie est un constat. Elle présente une description de 1 'environnement, de 1 'entreprise par ses structures, par son processus de transformation, par ses sous-systèmes et par son climat organisationnel. Des zones problématiques sont identifiēes. 
Lors de la troisième partie, le chercheur élabore une solution. A partir d'un point de vue et d'un modèle idéalisé, il procède à l'analyse de la situation réelle. Des points forts et faibles sont déterminés et les recommandations sont énoncées.

Les deuxième et troisième parties constituent un diagnostic organisationnel. Dans la quatrième partie, nous évaluons le degré d'atteinte des objectifs de la recherche. 
PREMIERE PARTIE

PRESENTATION DE LA RECHERCHE 


\section{INTRODUCTION}

Cette première partie informe sur la recherche. Elle introduit le lecteur à la problématique et à l'entreprise. Les objectifs de la recherche sont indiquēs et constituent les "out put" désirés. Les précisions sur la méthodologie situent le lecteur quant à la façon, pour le chercheur, de procéder pour la réalisation de la recherche. 


\subsection{CONTEXTE DE LA RECHERCHE}

La recherche s'est effectuée dans un contexte particulier. Il s'agit d'un cas d'intégration de la recherche et de l'action à l'occasion d'une expérience d'entrepreneurship.

\subsubsection{Le chercheur-acteur}

Après une période d'emploi de 12 annëes dans le secteur public, le chercheur se retrouve simultanément étudiant au programme de maîtrise en gestion des petites et moyennes organisations à l'Université du Québec en Abitibi-Témiscamingue et entrepreneur all sein d'une compagnie privée d'exploration en voie de devenir une entreprise publique.

\subsubsection{L'expérience d'entrepreneurship}

Il faut considérer la période de décembre 1985 à décembre 1986 comme étant la période de création de l'entreprise. C'est au cours de cette période que l'entreprise est passée du statut d'entreprise privée au statut d'entreprise publique. Durant cette période, l'entreprise est devenue opérationnelle avec des ressources humaines, financières et physiques. Cette période débute avec l'incorporation en décembre 1985 et se termine avec l'inscription en bourse en décembre 1986 .

\subsubsection{Programme de maîtrise en P.M.0.}

Le programme vise la formation d'analystes spécialisés dans le diagnostic organisationnel et permet aux étudiants d'intervenir dans le milieu par la création d'une entreprise. C'est la deuxiēme 
orientation qu'a choisie l'étudiant lors de son arrivée au programme de maitrise en septembre 1985.

\subsubsection{Mémoire de recherche-action}

En plus de la création d'entreprise, avec l'accord du directeur de programme, il fut convenu que l'étudiant produise un mémoire de recherche-action portant sur 1 'entreprise. Il fut décidé que 1 'étudiant effectue un autodiagnostic sur une problématique que le chercheur-acteur découvrait au cours de la mise sur pied de son entreprise. Le mémoire de recherche-action permet une réflexion therfectuelle sur certains concepts et augmente le niveau des connaissances sur 1 'exploration minière.

\subsubsection{Travaux de base}

Il fut décidé d'intégrer la majorité des travaux produits par 1 'étudiant lors de sa scolarité. Ces travaux pouvaient être ainsi en relation avec le mëmoire de recherche-action. Cette approche a permis à 1 'étudiant de réaliser trois travaux prémémoires sur des problèmes que connaissait l'entreprise. Ces travaux ont permis au chercheur d'exécuter le gros du travail de recherche durant cette përiode. 


\subsection{L'ENTREPRISE}

Exploration minière Normétal inc. est une compagnie publique "junior" d'exploration. Normétal inc. fut incorporée en décembre 1985. Depuis cette date, pour assurer son financement, l'entreprise a réal isé un placement privé de 170000 \$ et une souscription publique de 1530000 \$. L'entreprise est inscrite à la cote de la Bourse de Montrēal depuis le 19 dēcembre 1986 (NML -Bourse de Montréal).

Les actifs de 1 'entreprise sont des terrains miniers détenus à $100 \%$ et sous option et des placements. Parmi ces actifs, nous retrouvons six propriétés minières. La propriété Normétal compte 13600 acres de terrain (2 concessions minières et les droits de surface), un résidu minier de plus de 9 millions de tonnes. La propriété Barraute compte 1500 acres. Récemment, l'entreprise a acquis la propriété La Sarre, laquelle compte 34000 acres. Plus récemment, elle a acquis les propriētēs Bassignac, Brouillan et Dalquier, totalisant 5100 acres.

Cette entreprise réalise des activitēs d'exploration pour les métaux précieux et de base. En association avec un partenaire, elle procède à une étude de faisabilité sur son résidu minier. Les travaux d'exploration s'exécutent en continu depuis mars 1986.

Trois terrains miniers sont explorés en coparticipation avec Exploration La Sarre inc. Celle-ci est une filiale de Exploration Normétal inc. et elle est gérée par la même équipe. El le peut ainsi gagner un intérêt dans les trois propriétés Normétal, Barraute et La Sarre. Elle sera inscrite en Bourse de Montréal dès le début de 1988. A noter également qu'elle a réalisé une émission publique de $2630000 \$$ en 1987 . 
Les ressources humaines de 1 'entreprise totalisent 10 employés permanents. Elle utilise les consultants et contracteurs pour ses autres besoins de main d'oeuvre spécialisée.

\subsection{LA PROBLEMATIQUE}

Depuis les années 70 , les travaux d'exploration étaient surtout financés à même les revenus de production des compagnies productrices et aussi par le produit de la vente du capital-actions des compagnies minières sans revenus de production.

Dès 1980, 1'avènement des actions accréditives et des sociētés en commandite ainsi qu'un traitement fiscal privilègié modifient le financement de 1 'exploration. Avec ce système, l'investisseur réclame $100 \%$ des dépenses d'exploration majorées de $331 / 3 \%$ au palier fédéral et de $662 / 3 \%$ au palier provincial. Le compagnie vend son capital-actions à l'investisseur, lequel réclame les dépenses d'exploration contre son revenu et profite ainsi d'une récupération fiscale en fonction du revenu imposable. L'investisseur réduit ainsi le coût net de son investissement tout à fait risqué.

Dans ce système, la compagnie s'engage à dépenser un pourcentage des argents recueillis en frais d'exploration. Généralement, $80 \%$ des argents servent aux frais d'exploration et $20 \%$ aux frais d'administration. Il en résulte que la compagnie a un problème perpétuel de fonds de roulement. Elle ne peut pratiquement pas garder dans son trésor des argents en prévision des aléas. En fait, elle est obligée de se financer perpétuellement par la vente de son capital-actions. Les actionnaires connaissent ainsi une dilution de plus en plus importante sans augmenter la solidité financière de leur compagnie. 
Un tel système et une amélioration de la réglementation concernant les compagnies minières (actions de fondation, fonds de roulement, capitalisation, etc.) ont favorisé l'émergence d'un marché primaire. Nous assistons à une prolifēration de compagnies "juniors" qui financent des travaux d'exploration importants. Les maisons de courtage exécutent de plus en plus de souscriptions publiques.

Les conséquences de ce système sont multiples. La prolifération des "juniors" et la rapidité de leur mise sur pied pose un problème de qualité, de compétition et de survie. L'abri fiscal crée une mentalité chez l'investisseur qui ne facilite pas le marchē secondaire et pose de façon plus aiguë le problème de la qualité et de la survie.

Les maisons de courtage exerçaient un certain contrôle sur la qualitē des compagnies à financer au début du programme. Il y a un relâchement sur ce contrôle informel en raison du marché extraordinaire que les maisons de courtage peuvent s'accaparer. Donc, des produits de qualité variable sont offerts au public investisseur.

C'est dans cette problēmatique globale qu'Exploration miniēre Normétal inc. doit assurer sa survie.

Trois éléments sont nécessaires pour assurer la qualité d'une "junior": l'expertise technique, la propriēté et la promotion. Ceci constitue 1 'essentiel de la planification stratégique de Normétal inc.

Il faut entendre par expertise technique, la nature et le niveau des connaissances (géologie, géophysique, finance, gestion, etc.) ainsi que les succès antérieurs (découvertes).

Du point de vue financier, l'entreprise doit vendre constamment de son capital-actions pour s'assurer que son fonds de roulement soit 
suffisant pour exécuter les travaux d'exploration et pour conserver une stabilité à ses ressources humaines.

Les ressources humaines au service de Normétal inc. peuvent-elles constituer un actif supplémentaire à sa pérennité? Quelles sont les forces et faiblesses des ressources humaines de 1 'entreprise?

De quelle façon les éléments d'actifs peuvent-ils contribuer à 1 'augmentation de la stabilité? Comment la promotion peut-elle servir cette entreprise?

\subsubsection{Evolution de la problématique}

Récemment, le gouvernement fédéral annonçait des changements importants à la fiscalité quant au traitement des gains en capital et des abris fiscaux. Les changements annoncés auront comme conséquence de ramener l'industrie de l'exploration à la situation qui prévalait dans les années 70. Donc, le problème de survie se pose de façon accrue.

Normétal inc. a procédé à la création d'une filiale pour éviter de façon momentanée le problème de la dilution du capital et lui permettre d'obtenir des fonds pour l'exploration de ses propriētés. Cette dēcision permet de régler à court terme la problématique de survie de 1 'entreprise.

\subsection{TYPE DE RECHERCHE}

Cette recherche est une recherche-action. Elle implique 1 'existence simultanée d'une recherche et d'une action, soit la participation du chercheur et de l'acteur. 


\begin{abstract}
La recherche est une investigation originale entreprise en vue d'acquērir de nouvelles connaissances scientifiques et une meilleure compréhension des phēnomènes ou en vue d'acquérir de nouvelles connaissances pour les appliquer à la résolution de problèmes techniques ou pratiques. L'action est un ensemble de gestes produits en vue de transformer de façon intentionnelle une situation préexistante dans le but de 1 'amél iorer'.
\end{abstract}

Le chercheur est impliqué dans la propriété et la gestion de l'entreprise. Son rôle de chercheur vient ajouter plus de rigueur intellectuelle à sa démarche et augmenter les connaissances de la problématique de l'entreprise. Ce travail de recherche est l'une des premières productions scientifiques sur le phēnomène des Junior. Le chercheur a voulu cerner une problëmatique globale et en trouver une application concrète à son entreprise.

\title{
1.5 OBJECTIFS DE LA RECHERCHE
}

En plus de la mise sur pied d'une entreprise, la recherche a pour objectif d'augmenter les connaissances tant administratives que sur 1 'entreprise el le-même.

L'objectif d'augmentation des connaissances de l'entreprise se réalise par un travail d'autodiagnostic. Le chercheur, en raison de ses fonctions et de son implication dans 1 'entreprise, est responsable de l'orientation stratégique de 1 'entreprise. Il exerce cette responsabilité envers le conseil d'administration et l'assemblée des actionnaires. En rēalisant une étude sur la planification

1. Paul Prévost, Le diagnostic-intervention: une approche systémique au diagnostic organisationnel et à la recherche-action, Chicoutimi, 1983, Département des sciences économiques et administratives. 
stratégique, Te chercheur-acteur satisfait les objectifs individuels de 1 'acteur et ceux de 1 'entreprise.

L'objectif d'augmentation des connaissances se réalise par la vērification de l'applicabilité de certains concepts administratifs usuels à l'industrie de l'exploration.

\subsection{METHODOLOGIE}

Le cadre méthodologique d'une recherche spēcifie les mēthodes ut il i sées.

Ce mémoire de recherche est issu de la méthodologie du diagnostic et de la méthodologie des systèmes souples de Peter Chekcland.

Le diagnostic compte les quatre ètapes suivantes:

1- cueillette des données;

2- description de la réalité;

3- èlaboration d'un modèle en fonction de T'organisation et de $l$ 'intervenant;

4- choix des solutions.

Le cadre méthodologique de notre recherche emprunte certains éléments à la méthodologie des systèmes souples pour enrichir la mëthode du diagnostic. Il comprend les ëtapes suivantes:

1- cueillette des données;

2- description de la réalité par la description de l'environnement, de la structure, du processus de transformation, du climat, des sous-systèmes et de la problëmatique; 
3- choix d'une perspective d'analyse et conception d'un modèle pour analyser la situation problématique;

4- comparaison du modèle avec la réalité et choix des zones problématiques;

5- choix des solutions et recommandations. 


\section{CONCLUSION}

Lors de la production d'un travail de recherche, il importe que sa scientificitē puisse être reconnue. Cette validation de la recherche est fonction de la mēthodologie. Cette partie du travail fournit les données nécessaires et les paramètres pour évaluer la recherche. 
DEUXIEME PARTIE

ETAT DE LA SITUATION 


\section{INTRODUCTION}

Cette partie du mémoire est essentiellement descriptive. Elle ne contient aucune analyse, elle constitue un constat. Il s'agit d'une présentation de 1 'entreprise dans son environnement, ses structures, ses ressources, ses principales fonctions, sa raison d'être et son climat organisationnel. Elle constitue une "image enrichie" d'une quantité d'informations qui facilitent la comprēhension de l'entreprise et de sa problématique globale. A la fin de cette partie, le focus est mis sur une zone problématique et elle est décrite. 


\subsection{L'ENVIRONNEMENT}

\subsubsection{Les actions accréditives}

\subsubsection{1 description}

Le système de financement par les actions accréditives est en place depuis 1980. Les gouvernements fédéral et provincial ont mis sur pied ce programme pour réanimer l'industrie de l'exploration minière, devenue moribonde à l'époque. Ce programme est en fait une mesure fiscale pour réaliser des programmes d'exploration.

Les actions accrēditives proviennent de dispositions fiscales qui donnent à l'investisseur la possibilité de rêduire son impôt par la déduction permise pour des dépenses d'exploration effectuées pour son compte, au Canada et au Québec, dans le secteur des ressources naturelles'.

Au début du programme, l'investisseur du Québec pouvait déduire dans le calcul de son revenu provincial pour fins d'impôt $1662 / 3 \%$ de son investissement et $1331 / 3 \%$ pour fins d'impôt fédéral. Depuis 1987, l'investisseur du Québec ne peut déduire que $1331 / 3 \%$, comme les autres canadiens.

Depuis juin 1987, le gouvernement a accepté de maintenir des actions accréditives à $100 \%$. Le système va connâ̂tre une élimination progressive d'une partie de la déduction pour épuisement gagné, laquelle déduction était ajoutée à 1 a déduction de $100 \%$. Ces changements s'inscrivent dans le cadre de la réforme fiscale.

1. Le financement public des entreprises de ressources, Bourse de Montréal, 1985. 


\subsubsection{2 base du système accréditif}

Avant l'instauration du système accréditif, les compagnies minières pouvaient reporter (accumuler) les dépenses d'exploration et appliquer ces dépenses d'exploration (frais reportés d'exploration) contre des revenus futurs de production.

Avec le système accréditif, les compagnies minières peuvent (à leur choix) ne plus reporter les dépenses d'exploration (accumuler) pour les appliquer contre des revenus futurs de production. Elles transfërent ce droit à l'investisseur, lequel peut alors appliquer des dépenses d'exploration contre ses revenus. Le jour où la compagnie minière aura des revenus de production, elle n'aura pas de frais d'exploration reportés pour les appliquer contre ses revenus. Il est connu que les opérations minières sont très lucratives, le rendement sur le capital investi étant élevé. Donc, l'entreprise minière paiera des impôts dès le début de la production.

\subsubsection{3 fonctionnement}

L'investisseur dépose ses fonds auprès d'un fiduciaire. Le fiduciaire transfère ces fonds à la compagnie émettrice pour financer ses programmes d'exploration. Une fois le programme d'exploration terminé, l'investisseur reçoit des actions ordinaires de la compagnie émettrice qu'il peut alors revendre grâce au marché boursier. Le nombre d'actions reçues est fonction d'une entente entre la compagnie et $l$ 'investisseur.

Les dépenses admissibles à des frais d'exploration sont "des dëpenses encourues pour déterminer l'existence, la location, 1 'étendue et la qualité d'une ressource minérale" et pour l'amener au stade de l'exploration. Ces dépenses doivent être encourues dans un délai prescrit. 
FIGURE I: ENVIRONREMENT

SYSTEME FINANCEMENT

SYSTEME ACCREDITIF

MARCHE ET PRIX METAUX

\begin{tabular}{|c|c|}
\hline RESSOURCES HUMAINES & PRODUCTION \\
\hline $\begin{array}{l}\text { - Conseil d'administration } \\
\text {-Direction } \\
\text { - Personnel }\end{array}$ & $\begin{array}{l}\text {-Cumul d'informations } \\
\text { géologiques } \\
\text {-Confirmation ou information } \\
\text { de } 1 \text { 'existence et de dépôts } \\
\text { minéralisés }\end{array}$ \\
\hline FINANCE & MARKETING \\
\hline $\begin{array}{l}\text { - Contrôle financier } \\
\text {-Financement (entrée de fonds) } \\
\text { - Rapports financiers }\end{array}$ & $\begin{array}{l}\text {-Vente lors d'émissions } \\
\text { publiques } \\
\text { Mousser l'intērêt des inves- } \\
\text { tisseurs pour l'entreprise }\end{array}$ \\
\hline
\end{tabular}

FOURNISSEURS CONSULTANTS

\section{CLIENTS}

MARCHE BOURSIER
ASSEMBLEE DES ACTIONNAIRES

PHYSIQUE

TERRAINS

TECHNOLOGIE

MAJEURS

IRSE DE MONTREAL COMMISSION DES VALEURS MOBILIERES 


\subsubsection{4 traitement fiscal}

La compagnie ne réclame pas ses frais d'exploration contre ses revenus et permet à l'investisseur de le faire à sa place. Ce traitement fiscal généreux devient un abri fiscal.

Les frais d'exploration au Canada (F.E.C.) sont déductibles à $100 \%$ au gouvernement fédéral et provincial. A cette déduction s'ajoute une déduction supplémentaire pour épuisement pour l'exploration miniēre. Cette déduction est égale à $331 / 3 \%$ des frais d'exploration au Canada (F.E.C.). Donc, le total des déductions possibles est de $1331 / 3 \%$ car les deux types de déductions sont cumulatives. Bien entendu, les dépenses d'exploration sont dēfinies et décrites.

Le ministre des finances du Canada, monsieur Wilson, a modifié le traitement fiscal du système accréditif en juin 1987. Ainsi, l'élimination progressive de la déduction pour épuisement diminuera les avantages fiscaux.

$\begin{array}{ll}\text { Jusqu'au } 30 \text { juin } 1988 & : 1331 / 3 \% \\ \text { Du ler juillet au } 30 \text { juin } 1989 & : 1162 / 3 \% \\ \text { Après le } 30 \text { juin } 1989 & : 100 \%\end{array}$

Lorsque l'investisseur dispose de ses actions, le produit de la vente devient un gain en capital et est imposé à $50 \%$ selon les règles d'imposition en vigueur. Une perte en capital ne peut être créée.

Dans la récente réforme fiscale du ministre Wilson, l'augmentation du taux d'imposition des gains en capital va avoir un effet indirect important:

Jusqu'au 31 décembre $1987 \quad: 50 \%$ 


$$
\begin{array}{ll}
\text { Jusqu'au } 31 \text { décembre } 1987 & : 50 \% \\
1988 & : 662 / 3 \% \\
1989 \text { et suivantes } & : 75 \%
\end{array}
$$

Un changement important dans cette rëforme est que les dēductions attribuēes à une action accréditive augmentent les pertes de placement, de sorte qu'un investissement dans les actions accrëditives pourrait réduire l'exonération des gains en capital de $100000 \$$.

\subsubsection{5 avantages des actions accréditives}

Ces actions accréditives permettent des économies d'impôt pouvant atteindre $80 \%$ de la somme investie selon que la compagnie émettrice ne veuille pas recueillir des fonds pour son administration. Il arrive qu'une partie de l'investissement se fasse en actions ordinaires et accrēditives à des pourcentages variables. Le coût net d'acquisition des actions (1e coût payé - les économies d'impôt) se trouve considérablement atténué grâce à l'abri fiscal. Le rendement sur le capital investi (coût net) est généralement positif pour 1 'ensemble de l'industrie. L'achat d'actions accréditives est ainsi devenu un abri fiscal des plus populaires au Canada. 


\section{EXEMPLE: TAUX IMPOSITION MAXIMUM $64000 \$$ (Avant changement de juin 87)}

FEDERAL (32.8\%) PROVINCIAL (27.2\%) TOTAL

Investissement
$10000 \$$
Traitement fiscal

$133 \%$

$13300 \$$

$13300 \$$

Economie impôt

$4362,40 \$$

$3617,60 \$$

$7980 \$$

Coût net de

l'investissement

$10000 \$-7980 \$$ (économie $=2020 \$$

Rendement

Valeur au marchē de l'investissement -prix actions Coût net

Investissement $=$ dëboursē initial de 1 'investisseur.

Traitement fiscal $=$ le total des déductions possibles transférées à 1 'investisseur grâce au système accréditif.

Economie d'impôt $=$ impôt économisé en fonction du taux marginal d'imposition.

Coût net de l'investissement

dëboursē réel après que l'investisseur ait obtenu sa récupération fiscale.

Rendement $\quad=$

taux de rendement produit en appliquant la valeur au marché de l'investissement sur le coût net de 1 'investissement. 
Ces récents changements annoncés à la fiscalité fédérale vont avoir un effet important sur les avantages des actions accréditives.

L'épargne d'impôt d'un investissement dans les actions accrēditives sera moins importante qu'aurapavant. De plus, il existe une forte probabilité que la disposition des actions accréditives entraînera un gain en capital non exonéré.

De plus, l'investissement dans les commandites sera moins intēressant, car les frais d'émission et les honoraires des mandataires seront déductibles sur une période de cinq années.

L'économie d'impôt amoindrie et l'imposition du gain en capital pour les actions accréditives vont modifier le coût net et le rendement sur 1 'investissement de façon drastique et rendre ce type d'investissement moins intēressant.

\subsubsection{Les commandites et autres}

Une société en commandite est une forme d'organisation juridique qui peut être utilisée dans le système de financement accrēditif. Les avantages fiscaux reliēs aux actions accrēditives sont aussi possibles dans les sociétés en commandite.

On utilise la société en commandite pour des levées de fonds importantes. Il y a généralement un partenaire passif (le commanditaire) et un gérant (le commandité).

Le financement par 1 'intermédiaire d'une société en commandite sera moins intéressant, compte tenu que la rēforme du ministre Wilson précise que les frais d'émission et les honoraires du mandataire sont déductibles sur une période de cinq années. 


\section{diversité d'application}

Avec la demande d'abris fiscaux, le système accréditif permet différentes formes de financement. L'idée maîtresse qui a guidé l'évolution est la diversification. C'est ainsi que sont apparus les fonds miniers (similaires aux fonds mutuels). Une autre sophistication est apparue, soit la possibilitē pour l'investisseur, ou plutôt la commandite, de gagner un intérêt dans le projet au lieu de recevoir des actions.

Le tableau suivant résume les différentes possibilités avec leurs principales caractēristiques. Quelques exemples sont citēs. 
TABLEAU I : APPLICATION DU SYSTEME ACCREDITIF

\begin{tabular}{|c|c|c|c|}
\hline $\begin{array}{l}\text { COMMANDITE POUR } \\
\text { PLUSIEURS COMPAGNIES }\end{array}$ & $\begin{array}{l}\text { COMMANDITE POUR } \\
\text { UNE COMPAGNIE }\end{array}$ & FONDS MINIER & $\begin{array}{c}\text { FINANCEMENT } \\
\text { ACCRED ITIF } \\
\text { NORMAL } \\
\end{array}$ \\
\hline
\end{tabular}

\section{-Les commanditaires reçoivent une quantité d'actions des différentes compagnies sup- portées par la commandite.}

-Le nombre d'actions varie en fonction du prix de l'action de la compagnie junior.

-Abri fiscal moyen de $85 \%$.

-Souscription minimum et maximum.

-Grande diversification.

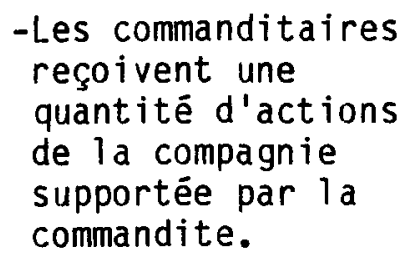

- Le nombre d'actions dépend du prix de de l'action.

-Abri fiscal moyen de $85 \%$.

-Souscription minimum et maximum.
-L'investisseur reçoit -Actions ordinaires des actions du fonds.

-Abri fiscal moyen $85 \%$.

-Abri fiscal moyen de $75 \%$ à $80 \%$ si admissible au R.E.A.Q.

-Fonds acquiert soit des participations

-Souscription minimun et maximum. indivises dans les propriētés supportēes, soit des actions des compagnies.

-Bons de souscriptior

-Bons de souscription. -Grande diversification.

\begin{abstract}
-Société en commandite de la Rivière Bell.

-Ressources Eider inc.

-La Pause inc.

- Cal iente inc.

-Midnapore inc.
\end{abstract}

-Société en commandite Ressources Rouyn 1986.

-Ressources Rouyn inc.
Cogesco inc. (intérêt indivis)

Q-Vest inc. (actions)
Forbex inc. $(80 \%)$

Exploration minière Normëtal inc. (R.E.A.Q. $75 \%$ ) 


\subsubsection{Le marché boursier}

\subsubsection{1 définition}

"La bourse c'est d'abord et avant tout un marché; donc un lieu organisé dans le but de favoriser l'échange. C'est un lieu de rencontre entre des vendeurs et des acheteurs possēdant le même langage mais des objectifs ou des conventions différentes. C'est donc un marchē d'enchères où se transigent des produits financiers"1.

La loi de l'offre et de la demande régit les échanges. Le marchē a pour but de faciliter la négociation des titres par 1 'ordonnancement et la standardisation.

\subsubsection{2 bourses}

Au Canada, il existe cinq bourses reconnues: Montréal, Toronto, Winnipeg, Calgary et Vancouver. Les titres négociés en bourse sont: les droits de souscription, les droits d'achat, les options, les contrats à terme et les actions. Chaque bourse accepte des titres inscrits à sa cote pour négociation. Chaque bourse permet à ses membres de négocier sur le parquet les titres inscrits à sa cote.

\subsubsection{3 marchē primaire}

Le marché primaire est constitué par la première offre d'une compagnie aux épargnants. Le produit de la vente est remis à la sociētē émettrice, moins les frais de courtage. Les titres sont offerts au moyen d'un prospectus. Les maisons de courtage offrent

1 Gérard Bérubé, Initiation aux valeurs mobilières, Publifor inc., 1984 , p.11. 
les titres aux épargnants selon deux façons: la prise ferme et la prise pour compte.

La prise ferme signifie que le courtier achète l'émission (titre émis) et la revend à ses clients. La prise pour compte signifie que le courtier s'engage à faire les meilleurs efforts possibles pour vendre les titres aux épargnants.

\subsubsection{4 marchē secondaire}

Sur le marché secondaire, le jeu de l'offre et de la demande fixe le prix de l'action. Les transactions (achats de titres) interviennent entre les investisseurs eux-mêmes et non entre l'émetteur et 1 'épargnant. Le produit de la vente est versé au vendeur, moins les frais de courtage. Ces derniers sont partagés entre l'acheteur et le vendeur.

\subsubsection{5 maisons de courtage}

Les concepts de marchē primaire et secondaire nous obligent à considérer l'état de 1 'industrie du courtage par rapport à 1 'industrie minière.

Au Québec, il existe à peu près une dizaine de maisons de courtage qui font des émissions de compagnies minières dans le marché primaire. Les "super-maisons" de courtage avec des réseaux de succursales à travers le Québec (Lévesque et Beaubien, McLeod Young and Weir, Geoffrion Leclerc) ne font que des ëmissions très importantes.

Les maisons de courtage, sans rēseaux de succursales au Québec, avec des courtiers en nombre variable, font des financements miniers. Les gros réseaux de courtiers sont intéressés à s'associer à des 
projets sûrs. Ils encourent sensiblement les mêmes frais avec une émission de dix millions de dollars qu'avec une émission d'un million de dollars. Les petites maisons de courtage "font les émissions des juniors", mais utilisent souvent les réseaux de vente des gros courtiers.

La vente s'articule au moyen d'un système d'allocation. Les courtiers des maisons de courtage travaillent uniquement à commission. Ainsi, une maison de courtage divise une émission entre ses courtiers individuels. Les courtiers, s'ils réservent des montants d'actions, les achètent et, par conséquent, doivent les vendre. Si la maison de courtage n'a pas assez de courtiers individuels pour vendre l'émission, elle collabore avec deux ou trois autres maisons pour vendre l'émission. Lors de semblables collaborations, les autres maisons font "des réservations qui sont revendues à leurs courtiers individuels", sel on le principe décrit.

Nous incluons une liste de toutes les maisons de courtage en opēration au Québec (annexe I). Le tableau suivant synthêtise l'industrie du courtage dans les financements miniers. Nous énumérons quel ques exemples de maisons de courtage. De plus, nous citons des exemples de maisons "faisant du minier"; il existe cependant d'autres courtiers qui en vendent. 
MAISONS DE COURTAGE AVEC SUCCURSALES

A TRAVERS LE QUEBEC
MAISONS DE COURTAGE SANS SUCCURSALE A TRAVERS LE QUEBEC
Lévesque, Beaubien inc. Geoffrion, Leclerc inc. McLeod, Young, Weir 1td McNeil, Mantha inc.

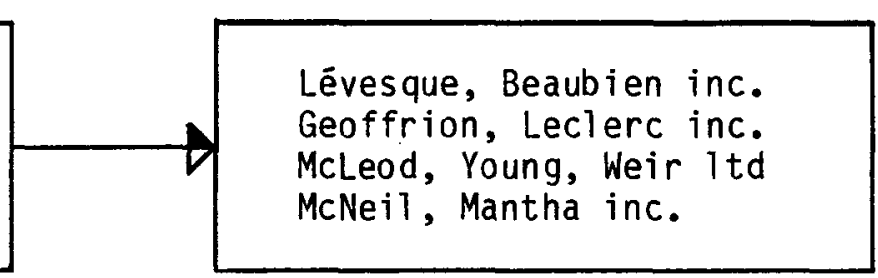

GROS COURTIERS 20 courtiers

Osler inc. Deacon, Hodgson inc.
Entre

5 et 20

courtiers

Deragon, Langlo is 1 td

Bel1, Gouinlock 1td

$\begin{array}{ll}\text { MOYENS COURTIERS } & \text { Entre } \\ & 5 \text { et } 20 \\ \text { Deragon, Langlois 1td } & \text { courtiers } \\ \text { Bel1, Gouinlock 1td } & \end{array}$

PETITS COURTIERS

Moins de

Deleeuw et associēs

5 courtiers

Soficorp inc.

N.B. La nomenclature choisie est basée sur la géographie et sur le nombre de courtiers individuels. 


\subsubsection{Marchē des métaux}

\subsubsection{1 l'industrie canadienne et mondiale}

L'industrie minière canadienne traverse une période difficile. La plupart des métaux sont en surplus d'inventaire. Il s'agit d'un phénomène mondial. Les pays du tiers monde et en voie de développement exportent la presque totalité de leur production. Au Canada, nous exportons $80 \%$ de notre production. Donc, notre marché domestique n'est pas suffisant. Nous devons compétitionner sur les marchés d'exportation. Il s'ensuit une pression à la baisse sur les prix. L'industrie doit augmenter sa productivité par des changements technologiques, des nouveaux équipements pour maintenir sa compétitivité et maintenir un taux acceptable de rendement sur le capital.

\subsubsection{1'activité économique}

Les métaux de base sont frappés par une activité économique mondiale fonctionnant au ralenti. Les inventaires sont souvent croissants et 1 'équilibre entre 1 'offre et la demande est difficilement maintenu. A la suite de cette diminution de la consommation, les entreprises minières se retrouvent avec des capacités de production en réserve. Le recyclage et 1 'ut ilisation de substituts contribuent à 1 'augmentation de cette capacité inutilisée.

\subsubsection{3 les métaux précieux}

Le secteur des métaux précieux se porte bien. La production de l'or est en croissance et de nombreux producteurs émergent. Le prix de l'or est en progression constante depuis le dëbut des années 70 . Cette hausse constante et soutenue permet la rentabilité de projets marginaux. De nombreux facteurs expliquent la hausse du prix de 
l'or: les pressions inflationnistes, la baisse du dollar américain.

\subsubsection{4 effets sur T'exploration}

Le tempo de 1'exploration n'a pas ralenti. C'est seulement la direction de 1 'exploration qui a changée. Aujourd'hui, on explore presque exclusivement pour les métaux précieux. Ce sont les seuls métaux qui offrent des prix èlevēs et des rendements sur le capital investi intēressant.

Quel ques entreprises minières avec des infrastructures de transformation (smelters) continuent 1 'exploration pour les métaux de base, uniquement pour approvisionner leurs infrastructures. Citons Noranda inc. et Finneth inc.

\subsubsection{5 perspective}

L'industrie minière canadienne est cyclique. Un cycle favorable aux métaux de base est en préparation. Notre économie entre dans une phase d'expansion et de croissance globale favorisée par la baisse des taux d'intérêt et des dépenses pētrolières. La consommation va s'améliorer et, par le fait même, se refléter sur l'industrie des métaux de base.

\subsubsection{L'organisation de 1 'industrie de 1 'exploration}

\subsubsection{1 'industrie}

L'industrie de 1'exploration minière compte plusieurs acteurs. Les investisseurs, les spéculateurs, les courtiers, les analystes financiers, les journalistes financiers, les compagnies minières de 
production, les compagnies d'exploration, les prospecteurs individuels sont les principaux acteurs.

\subsubsection{2 compagnies d'exploration}

Les compagnies d'exploration, appelées "juniors", financent gēnēralement leurs travaux d'exploration de deux façons. La première consiste à vendre de leur capital-actions pour obtenir des fonds. L'autre façon consiste à conclure des ententes avec des compagnies "majeures" pour faire exécuter des travaux sur leurs terrains.

\subsubsection{3 catégories de juniors}

Dans les prochains paragraphes, nous présentons des dēfinitions conceptuelles élaborées par Coopers and Lybrand dans une étude sur les pratiques comptables des compagnies minières en 1985 . Leur approche conceptuelle est basēe sur les activités et met en évidence le degré d'avancement des activités. En fait, il est évident qu'il existe plusieurs calibres parmi les juniors. Le tabieau suivant permet de visualiser la hiérarchisation. 
TABLEAU III: TYPES DE COMPAGNIES MINIERES

\section{NIVEAU D'AVANCEMENT}

ACTIVITE GENERALE

NIVEAU I

EXPLORATION

\section{ACTIVITES SPECIFIQUES}

Etudes géologiques
Etudes topographiques
Etudes géochimiques
Etudes géophysiques
Forages

\begin{tabular}{|c|c|c|}
\hline NIVEAU II & EVALUATION & $\begin{array}{l}\text { Evaluation de tonnage } \\
\text { Evaluation des méthodes } \\
\text { d'extraction } \\
\text { Evaluation métallurgi- } \\
\text { que } \\
\text { Etudes de marché, fi- } \\
\text { nancières et des infra- } \\
\text { structures. }\end{array}$ \\
\hline NIVEAU III & DEVELOPPEMENT & $\begin{array}{l}\text { Fonçage puits } \\
\text { Fonçage galeries } \\
\text { Excavations } \\
\text { Déblaiements }\end{array}$ \\
\hline NIVEAU IV & CONSTRUCTION & $\begin{array}{l}\text { Mise en place des } \\
\text { infrastructures } \\
\text { d'extraction, de } \\
\text { traitement, de } \\
\text { production } \\
\text { Mise en place d'infra- } \\
\text { structures énergétiques } \\
\text { et de service }\end{array}$ \\
\hline
\end{tabular}

Evaluation de tonnage Evaluation des méthodes d'extraction Evaluation métallurgique nancières et des infrastructures.

Fonçage puits

Fonçage galeries

Excavations

Déblaiements infrastructures d'extraction, de traitement, de production Mise en place d'infraet de service

NIVEAU $V$ PRODUCTION

Extraction quotidienne sur base commerciale Traitement sur base commerciale 
Cinq activités générales constituent la base de 1 'industrie minière. Nous allons en faire une description sommaire.

L'exploration est la recherche d'un dépôt minéralisē, duquel une exploitation commerciale est attendue grâce à une opération d'extraction. Les études topographiques, géologiques, géochimiques, géophysiques et les forages d'exploration sont les principales activités.

L'évaluation signifie la détermination de la faisabilité technique et de la viabilité commerciale d'un dépôt particulier. Ceci comprend la détermination du tonnage et de la teneur du dépôt, l'évaluation des méthodes d'extraction, des tests métallurgiques, des processus de traitement, des infrastructures requises, des ètudes de marchē et financières.

Le développement signifie l'établissement d'accès au dépôt et d'autres préparations pour la production commerciale. Ceci comprend le puits, les galeries, les excavations permanentes, les déblaiements de mort-terrain et le roc.

La construction signifie l'établissement d'infrastructures pour 1 'extraction, le traitement, le transport de la production (ëdifices, machinerie, équipement, infrastructures pour 1'eau, 1 'électricitē et les égouts).

La production concerne les activités quotidiennes nécessaires pour obtenir un produit vendable du dépôt sur une base commerciale. Ceci inclut 1 'extraction et le traitement avant 1 a vente.

Dans la pratique, toutes les activités décrites peuvent être exécutées simultanēment ou pas. En fait, il est évident que le 
degré d'avancement de la propriētē ou des propriētēs détermine le niveau d'avancement du projet.

\subsubsection{4 catégories de majeures}

Une compagnie minière "majeure" est une entreprise qui reçoit des revenus d'une production minērale sur une base commerciale.

Dans le niveau $V$, il existe plusieurs types de producteurs. Nous précisons deux de ces types par des exemples concrets. Des gros producteurs sont des entreprises, telles que Mines Noranda et Minerais Lac. Ces entreprises exploitent plusieurs gisements à travers le pays, soit directement ou par 1 'intermédiaire des filiales. Des petits producteurs sont des entreprises, telles que Muscocho Exploration qui exploite un gisement d'or à Montauban. Mine Abcourt inc. exploite un gisement de métaux de base à Barville. Entre ces deux extrêmes, nous retrouvons plusieurs possibilités.

\section{$2.1 .6 \quad$ Les acteurs}

\subsubsection{1 fournisseurs}

Les entreprises d'exploration utilisent beaucoup la sous-traitance ou la consultation à cause du haut niveau de spécialisation des activités et du coût des équipements. Les principaux fournisseurs de biens et services sont les services de courtage, les services de géologie et gēnie-conseil, les services de laboratoire, les fournisseurs de matériel et équipement minier, les entreprises de forage et les services de consultation en levés géophysiques et géochimi ques. 


\subsubsection{2 les investisseurs}

Les épargnants, les investisseurs et les spéculateurs sont les acteurs qui génērent le capital à l'entreprise d'exploration. Ces participants sont conseillēs ou influencēs par les analystes financiers, les journalistes financiers, les courtiers et autres aviseurs sur la qualité du placement. En fait, une compagnie d'exploration n'a pas de client auquel elle livre un produit. Son client est plutôt son actionnaire qui recherche la performance boursière ou le gain. Ce n'est qu'au stade de la production que l'entreprise minière possède un système client, c'est-à-dire lorsqu'elle vend une ressource minērale.

\subsubsection{3 les clients}

Dans le paragraphe sur les investisseurs, nous débutons 1'explication sur le système client. Il est important de rappeler qu'il n'y a pas de produit livré par la junior. Son actionnariat s'apparente au système client. En fait, l'actionnaire participe à 1 'entreprise. A cause du système de financement (fonds génērés par la vente du capital-actions et non d'un profit de vente), il devient un acteur important à satisfaire pour assurer la pérennité de 1 'entreprise.

L'actionnaire se recrute sur les marchés "primaire et secondaire". Le marché primaire est constituē des premiers actionnaires au moment où $l$ 'entreprise offre ses titres au public pour la première fois. Sur ce marché, l'investisseur est d'abord intēressé par l'abri fiscal offert par le titre (système de financement accréditif).

Le marchē secondaire est constitué par l'échange des titres entre les acheteurs et les vendeurs lorsque le titre est inscrit en Bourse. L'attrait de l'abri fiscal n'existant plus, c'est le 
potentiel de l'entreprise qui retient l'attention. Par potentiel, i) faut entendre la performance boursière (appréciation du titre), le niveau d'avancement des projets (voir le tableau sur les catégories de junior) et. la situation financière totale de 1 'entreprise.

L'objectif ultime pour les acteurs est "une participation à une découverte, laquelle deviendra un actif qui produira des revenus soit une mine". Cet objectif ultime n'est pas atteint souvent statistiquement. Donc, l'industrie a des objectifs plus rapprochés. Une apprēciation du prix de 1 'action constitue un résultat "plus probable et plus rapproché", c'est aussi le produit.

Depuis quel ques années, l'industrie de 1'exploration est considérée comme un abri fiscal. Le traitement fiscal accordé aux investisseurs permet aux acteurs d'avoir un objectif plus immédiat, soit la récupération d'impôt.

Donc, lors de l'émission primaire, le client est un investisseur qui a besoin d'un abri fiscal. Sur le marché secondaire, le client devient le spéculateur. L'investisseur fidèle espère un profit et n'est pas préoccupé par les fluctuations boursières; il se recrute aussi dans le marché secondaire.

Le tableau IV résume les types d'actionnaires que l'entreprise retrouve à trois étapes diffērentes de son évolution. 


\section{TABLEAU IV : TABLEAU DES INVESTISSEURS}

\section{CYCLE DE VIE} DE L'ENTREPRISE

NAISSANCE

PRIMAIRE
MARCHE BOURSIER
TYPES D'INVESTISSEURS

Acheteurs d'abris fiscaux Hauts salariés principalement

Investisseurs amis à 1 'intērieur du placement privé

Spéculateurs à 1 a recherche d'un gain rapide

Investisseurs patients et intéressés à la croissance du prix de l'action

MATURATION SECONDAIRE

Investisseurs patients, actionnaires intéressés à la croissance du prix de 1 'action 


\subsubsection{Politique}

\subsubsection{1 période 1977-1987}

En 1977, un "comitē interministēriel" d'ētude sur le financement des compagnies d'exploration minière se penchait sur les difficultés de financement auxquelles faisaient face les "juniors". Selon le rapport de ce comité, le coût élevé d'une émission publique d'actions empêchait, plus souvent qu'autrement, le financement d'une entreprise ayant un programme d'exploration de moins de $250000 \$$. Un autre argument qui expliquait l'impopularité de ce type d'investissement était le manque d'incitatifs fiscaux face au capital de risque que représente le secteur de l'exploration minière.

Parmi les objectifs visēs à cette époque par le comité, il y avait la protection du public investisseur, le maintien du caractère indépendant et québécois de la petite prospection et l'amélioration de la disponibilité de capitaux de risque pour les "juniors". Dans ce contexte, le comité faisait quatre recommandations afin d'améliorer le financement des entreprises d'exploration minière:

1. regroupement des prospecteurs dans une association qui agit à titre d'interlocuteur permanent et efficace et devient un organisme susceptible de fournir à ses membres toutes les informations et le support nécessaire dans le cadre de leurs activitēs;

2. créer un fonds de développement minier susceptible d'apporter un financement d'appoint aux programmes d'exploration proposés par les prospecteurs ou par le gouvernement; 
3. modifier le cadre juridique des sociētēs en commandite de manière à permettre d'assouplir le mode de fonctionnement et faciliter ainsi le financement, par des fonds privés, de programmes d'exploration en assurant la déductibilité fiscale pour les associés;

4. faire examiner par le ministère des finances la possibilité d'accorder aux investisseurs, dans les titres d'entreprises d'exploration minière, des crédits d'impôt ou des déductions fiscales et de leur permettre d'entreprendre, le cas ëchëant, des consultations auprès du gouvernement fédéral pour que ce dernier adopte une position similaire sur cette question.

A la suite de la publication du rapport du comité, le gouvernement du Quëbec faisait amender le Code civil (Loi 75), à l'automne 1978, pour faciliter la formation des sociétés en commandite afin d'effectuer de l'exploration minière. Il encourageait, d'autre part, le regroupement des prospecteurs en subventionnant 1 'Association des prospecteurs du Quēbec (A.P.Q.). La Commission des valeurs mobilières du Québec commençait, quant à elle, à adoucir l'application de certains règlements dans le but de favoriser la vente des actions minières. Enfin, avec le Fonds de développement minier, le gouvernement du Québec acceptait de partager le risque financier de la prospection jusqu'à concurrence de $50 \%$ des frais d'exploration en échange de $33 \%$ de 1 a propriété, s'il y avait découverte.

Au niveau de la fiscalitē, les lois fédérales et québécoises permettaient, à cette époque, aux contribuables de déduire $100 \%$ des dépenses d'exploration contre les revenus de toutes sources. Une déduction additionnelle de $331 / 3 \%$ (déduction pour épuisement gagné) était permise uniquement contre les revenus provenant de ressources jusqu'à concurrence de 25\% (33 1/3\% au Québec) de ces revenus. 
C'est en 1980 que l'allocation additionnelle de $662 / 3 \%$ à l'égard des frais d'exploration, applicables aux particuliers, a étē introduite au Québec.

En 1980, le gouvernement du Québec, par l'entremise de son ministre des Finances, monsieur Jacques Parizeau, acceptait que "tout particulier membre d'une société d'exploration en commandite de minëraux, de pëtrole et de gaz, puisse en plus de toutes les dispositions actuelles soustraire de son revenu imposable $662 / 3 \%$ de ses frais d'exploration engagés au Quëbec". Cette disposition sera initialement valable pour les dépenses faites après le 31 mars 1980 et avant le ler janvier 1986.

En 1983, la fiscalité fédérale et provinciale change à bien des points de vue. Au niveau de la fiscalité des mines, un changement majeur est introduit:

1 a déduction pour épuisement gagné de $331 / 3 \%$ des dépenses d'exploration admises jusqu'à l'équivalent de 25\% (33 1/3\% au Québec) des revenus des ressources est désormais imputable contre les revenus de toutes sources (corporations et particuliers) et ce, pour les dépenses effectuées après le 19 avril 1983.

Ainsi, à compter de 1983, un particulier qui investit dans 1 'exploration minière au Quëbec peut avoir droit à des déductions de 133 $1 / 3 \%$ au fédéral et $1662 / 3 \%$ au Quëbec. Les corporations, elles, peuvent bénéficier de déductions maximales de $1331 / 3 \%$ aux deux paliers de gouvernement.

L'allocation additionnelle de $662 / 3 \%$ au Québec devait cesser le 31 décembre 1985. Toutefois, dans son budget du 23 avril 1985, le gouvernement du Québec annonçait une extension de la mesure jusqu'au 31 décembre 1987. Cette extension ne s'applique toutefois qu'à 
1 'égard d'une personne qui n'a pas de revenu d'exploitation minière. Tels sont les principaux changements institutionnels intervenus dans le financement minier depuis 1980.

La fiscalité encourageante des deux paliers de gouvernement est à la base du succès du programme accrēditif. Tout changement futur à la fiscalité sera déterminant. Donc, l'aspect politique du problème est important.

\subsubsection{2 état de la situation}

Il est évident que l'efficacité du système accréditif est redevable à une volonté politique. L'Association des prospecteurs du Quëbec est devenue un "lobby" important. Le même type de regroupement existe du côté anglophone. Les deux organisations se coordonnent pour l'exécution de leurs pressions sur les différents paliers de gouvernement.

Le fonds minier (subventions à 1 'industrie) a été modifié. Le ministère de l'Energie et des Ressources du Quëbec (M.E.R.) tente d'en faire bénéficier la Gaspésie actuellement, bien que certaines entreprises du Nord-Ouest quëbécois peuvent s'y qualifier encore.

La prēsente évaluation des effets de la réforme fiscale sur les actions accréditives est tirée d'une circulaire de Lacroix Frères co., consultants en fiscalitē et financement. L'évaluation précise les effets directs, indirects et les commentaires. Bien que les commentaires soient positifs, nous sommes d'avis qu'il faut attendre la réaction du marché boursier avant de se prononcer définitivement. 


\section{EFFETS DE LA REFORME FISCALE FEDERALE 1987 \\ SUR LES ACTIONS ACCREDITIVES}

\section{A. EFFETS DIRECTS:}

1. Maintien du système des actions accrëditives concernant les F.E.C. déductibles à $100 \%$.

2. Elimination progressive de la déduction pour épuisement gagné comme suit:

- jusqu'au 30 juin 1988 : $331 / 3 \%$

- du ler juillet 1988 au 30 juin 1989 : $162 / 3 \%$

- après le 30 juin 1989 : $0 \%$

3. La définition d'"actions accréditives" (actions non-admissibles à titre d'actions accrēditives) est élargie de façon à ce que les rachats d'actions ("buy back") ne soient plus admissibles.

\section{B. EFFETS INDIRECTS:}

1. Réduction des taux marginaux qui passeront, pour un résident québécois, d'environ 56,55\% en 1987 à 52,24\% en 1988 en supposant que le taux marginal québécois maximum de 1988 restera le même que celui de 1987 et en présumant un abattement du Québec égal à 16,5\% de 1 'impôt fédéral de base et une surtaxe de $3 \%$ de l'impôt fédéral de base.

2. Augmentation du taux d'imposition des gains en capital comme suit:

- jusqu'au 31 décembre 1987 : $50 \%$

. 1988 : $662 / 3 \%$

- 1989 et années suivantes : $75 \%$

3. Le budget propose que, après 1987, les gains en capital nets admissibles à 1 'exemption soient réduits par les pertes de placement déduites par le contribuable dans le calcul de son revenu. Les déductions attribuées à une action accréditive augmentent les pertes de 
placement, de sorte qu'un investissement dans les actions accréditives pourrait réduire l'exonēration des gains en capital.

Exemple: données:

- gain en capital

$3000 \$$

- intērêts-dêductibles

$1000 \$$

- déductions-actions accréditives

$1000 \$$

- intērêts-revenus $500 \$$

Compte tenu des autres paramètres fiscaux du contribuable, la situation aurait été la suivante:

- pour l'annẻe 1987

- gain en capital exonéré

- gain en capital imposable après exonération

- pour l'annēe 1988

- gain en capital imposable (3000 X 2/3)

(A) $2000 \$$ moins pertes de placement

- déductions-actions accréditives $1000 \$$

- intērêts-dépenses $1000 \$$

- intérêts-revenus

$(500) \$$

$1500 \$$

- gains en capital nets admissibles à l'exonération

(B) $500 \$$

- gain en capital imposable après exonération $(A)-(B)$

$\underline{1500 \$}$

4. Les frais d'émission pour émettre des titres (incluant les parts de sociétés en commandite) encourus après 1987 ne seront plus déductibles dans l'année de 1'émission, mais devront être amortis sur cinq ans. Les frais d'émission sont habituellement constitués de ceux de la 
commission de courtage, des honoraires légaux, des honoraires concernant la comptabilité et des coûts d'impression de prospectus.

\section{COMMENTAIRES:}

1. L'épargne d'impôt d'un investissement dans des actions accréditives sera moins importante qu'auparavant, comme le démontre le tableau cidessous:

$\begin{array}{ccccc}\text { F.E.C. engagés } & \text { Avant le } & \text { Entre le } & \text { Entre le } & \text { Après le } \\ & 31.12 .87 & 01.01 .87 & 01.07 .88 & 30.06 .89 \\ \text { et } 30.06 .88 & \text { et } 30.06 .89 & \text { et } 30.06 .89 & \end{array}$

Taux marginal

fédéral

maximum 1

$29,41 \%$

$25,08 \%$

$25,08 \%$

$25,08 \%$

Déduction

$133,33 \%$

$133,33 \%$

$116,66 \%$

$100,00 \%$

Epargne

d'impôt

$39,21 \%$

$33,44 \%$

$29,26 \%$

$25,08 \%$

De plus, il faudra considérer qu'il y a une forte probabilité que toute disposition d'actions accrēditives entrainera un gain en capital non exonérē, s'il y a dans la même année un investissement dans des actions accréditives.

2. Les compagnies d'exploration devront diminuer les primes incluses dans les prix d'émission d'actions accréditives.

3. Le financement par l'intermédiaire de sociētés en commandite sera moins intéressant qu'auparavant, considérant que les frais d'émission

1. En tenant compte de 1 'abattement du Québec remboursable de $16,5 \%$ (qui devrait être modifié) et de la surtaxe de $3 \%$. 
et les honoraires du mandataire ne seront plus déductibles en entier dans l'annēe de 1 'émission.

4. Avec une définition élargie des actions présentes, ce sera la fin probable des "buy back".

Malgré tout, les actions accréditives demeureront l'abri fiscal par excellence, attendu que les autres investissements avec saveur fiscale ont èté éliminés (M.U.R.B.) ou sont beaucoup moins attrayants (film canadien).

\subsubsection{Légal}

2.1.8.1 bourse de Montréal

Dans la section concernant l'aspect politique, nous avons vu qu'en 1977, la Bourse de Montréal a elle aussi assoupli ses rēgles envers les entreprises de ressources naturelles.

\subsubsection{2 exigences de la Bourse de Montréal}

La Bourse a modifiē ses exigences quant aux fonds requis pour poursuivre un programme d'exploration. Il n'est plus nécessaire que 7 'entreprise ait les fonds en mains propres. L'entreprise peut se trouver en instance d'obtenir les fonds nëcessaires et son inscription en Bourse est conditionnelle à l'obtention de ces fonds. C'est ce qui se produit lorsqu'elle réalise une émission de titres accréditifs pour mener des travaux d'exploration. Les principales exigences pour l'approbation conditionnelle sont:

- un programme d'exploration continue prēparé par une autorité technique compétente; 
- pour toutes les propriētés qui font 1'objet d'un programme d'exploration pour 1 'année en cours, un rapport préparé par un expert indépendant recommandant ledit programme;

- les rapports annuels des trois (3) derniēres années (si disponibles) et tous les rapports intērimaires publiés depuis le dernier rapport annuel;

- une copie du prospectus provisoire récemment déposé auprès de la C.V.M.Q., ou le plus récent prospectus ou document équivalent déposé dans une autre province canadienne;

- une copie de la plus récente liste des actionnaires, si disponible, certifiée conforme par le registraire;

- un chèque au montant de 300 \$ fait à 1'ordre de la Bourse de Montréal pour défrayer les coûts de l'étude de la demande. Ceux-ci seront applicables aux frais d'inscription initiale si la demande est approuvée.

Lorsque l'approbation conditionnelle sera octroyée, la Bourse de Montréal informera la compagnie de l'échéancier pour l'inscription et 1 a documentation additionnelle à fournir.

La Bourse a aussi modifié ses exigences sur le fonds de roulement et sur le capital émis:

\section{Fonds de roulement}

Une compagnie d'exploration minière doit avoir un fonds de roulement minimum de $250000 \$(350000 \$$ dans le cas d'une compagnie pētrolière) et suffisant pour complēter le programme d'exploration pour 1 'année en cours. 


\section{Capital émis}

Un minimum de 200000 actions d'une valeur au marché minimum de $350000 \$$ et détenues par au moins 200 actionnaires publics enregistrés. Chacun d'eux doit dētenir individuellement au moins un lot régulier.

Un lot régulier se définit comme suit:

- Actions d'une valeur de 10 cents à 99 cents: 500 actions

- Actions d'une valeur de 1 \$ et plus:

100 actions

2.1.8.3 actions de fondation et la réglementation de la Commission des valeurs mobilières du Québec

La C.V.M.Q. a assoupli ses règles pour la vente des propriétés et le remboursement des frais d'établissement.

Lors d'un premier financement public, la C.V.M.Q., la Bourse de Montréal et le courtier peuvent exiger qu'une partie des actions de fondateurs soit bloquée (entiercée) pour une certaine période de temps, dans le but d'assurer une juste mesure entre le risque et les efforts assumés par les fondateurs et le risque assumé par le public investisseur.

Les fondateurs des sociétés d'exploration de ressources naturelles ont droit de recevoir des actions libres de 7 'entiercement en remboursement des frais de premier établissement avancés. Des actions peuvent aussi être émises en contrepartie d'un terrain, mais ces dernières devront être entiercées.

Cette politique se résume comme suit:

1. Lors d'un premier financement public, les frais de premier 
établissement avancés à la compagnie par le promoteur sont remboursés de 1 a façon suivante:

a) en espèces après le placement;

b) en actions à un prix au moins égal au prix des actions vendues lors du placement;

c) une combinaison des deux modalitēs précédentes.

2. Des actions émises en contrepartie d'un terrain, généralement jusqu'à un maximum de $\mathbf{7 5 0 , 0 0 0 , ~ s e r o n t ~ e n t i e r c e ́ e s ~ e t ~ p o u r r o n t ~}$ être 1 ibērēes de 1 a façon suivante:

a) $10 \%$ du total dès 1 a fin de 1 'opération de placement;

b) $10 \%$ du total pour chaque exercice financier suivant le placement, jusqu'à concurrence des quatre exercices financiers selon les progrès réalisés sur la propriété appartenant à 7 'émetteur;

c) $20 \%$ du total lors de 1 a découverte d'un gi sement;

đ) la différence dès la mise en exploitation ${ }^{1}$.

La politique de la Bourse de Montréal sur les actions de fondateurs est parfaitement conforme à l'instruction générale No Q-4 de 1 a C.V.M.Q. La C.V.M.Q. a délēgué ses pouvoirs quant à la libération d'actions entiercées à la Bourse de Montréal lorsqu'une sociēté est inscrite à sa cote.

Ces changements ont suscité "l'entrepreneurship". Auparavant, 1 'individu ne pouvait espërer recevoir des contreparties aussi liquides pour les terrains et pour les frais d'établissement; les changements ont facilité le financement des projets.

1. Le financement public des entreprises de ressources, Bourse de Montréal, 1985. 


\subsubsection{Impact économi que}

Nous traitons la question de l'impact économique direct et indirect en nous inspirant de la récente étude de l'Association des prospecteurs du Québec, préparée par Sociēté-Conseil Maheu Noiseux et Roche Ltée Groupe-Conseił ${ }^{1}$.

Nous croyons que la qualité de cette étude et sa mise à jour récente sont des facteurs dominants pour justifier son utilisation.

\subsubsection{1 dépenses d'exploration F.E.C.}

Les sommes levées par le biais des actions accréditives et des sociétés en commandite se répartissent selon deux composantes. La majeure partie de ces financements $(80 \%)$ est versēe en dépenses d'exploration admissibles aux F.E.C. La fraction restante sert à défrayer Tes frais d'émission et d'administstration qu'impliquent ces financements publics. Le deuxième chapitre de l'étude démontre 1 'importance des épargnes canadiennes drainées par les actions accrêditives et les sociétés en commandite et dépensées au Québec. Cela représente un influx de capitaux de 315 millions de dollars courants dépensés au Québec au cours de la période 1980-1985. Les F.E.C. émis et dépensés au Quëbec se chiffrent à $202 \$$ millions nonactualisés, alors que les F.E.C. levés dans les autres provinces et dépensēs au Quēbec attteignent $61 \$$ millions. A ce montant, nous ajoutons des frais d'émission et d'administration des F.E.C. financēs et dépensés au Quēbec, soit $50 \$$ millions? 2

1. Etude d'impact économique, Loc. cit.

2. On présume que les frais d'émission et d'administration des émissions hors-Québec mais dépensés ici demeurent en dehors de la province. C'est-à-dire que ce sont des courtiers, comptables et avocats non résidents au Québec qui en bénéficient. 
Les tableaux $V$ et VI résument les impacts directs et indirects (en amont) de ces financements sur les F.E.C., les frais d'émission et d'administration 1 . Les simulations du modèle intersectoriel estiment à 3205 personnes-année l'emploi direct généré par ces F.E.C. au sein de l'industrie des services miniers, et à 1776 personnes-annēe 1 'emploi indirect engendré chez les fournisseurs de biens et services. En tout, 4981 personnes-année auraient oeuvré grâce à ces émissions. A cette main-d'oeuvre correspond une masse salariale de 97,8 \$ millions directs, 42,2 \$ millions indirects, pour un total avoisinant les 140 \$ millions demeurant directement et indirectement (en amont) en sol québécois sous forme de valeur ajoutēe. Les services miniers auraient versé directement $27,7 \$$ millions et indirectement 10.8 \$ millions en paiements fiscaux au gouvernement du Quëbec. De ces 38,5 \$ millions, l'impôt sur les salaires représente $48 \%$, la parafiscalité, $30 \%$, et les taxes indirectes, $22 \%$. Le gouvernement fédéral reçoit, pour sa part, directement et indirectement $22,6 \$$ millions, dont $58 \%$ en impôt sur les salaires, $28 \%$ en parafiscalité et $14 \%$ en taxes indirectes.

En tout, les états quëbēcois et canadien rēcupèrent $61,1 \$$ millions des 264,6 \$ millions dépensés en F.E.C., soit environ $24 \%$ de 1 a valeur ajoutēe simulēe par cette activité.

Les achats de biens et services des entreprises de prospection suscitèrent une activité indirecte en amont. On peut ēnumérer les principales industries qui en bēnēficient: les services miniers, les pièces mécaniques, les produits du pétrole, les explosifs et munitions. Il peut paraitre surprenant de retrouver ici des services dans les effets indirects, alors qu'ils sont compris dans

1. A moins d'indications contraires, tous les montants sont présentés en $\$ 1985$. 
les impacts directs. Nous sommes tout simplement en présence d'un phénomène d'autoconsommation, c'est-à-dire que les F.E.C. entraînent directement une activité au niveau des entreprises d'exploration (services miniers) qui les ont émises, et indirectement chez les entrepreneurs en forage lorsque cette tâche est confiée à contrat. Comme le forage et la prospection sont classés tous deux dans ces services, on assiste à un effet de rétroaction d'une activité sur el l e-même.

\subsubsection{2 autres dépenses}

Les frais d'émission et d'administration engendrent directement de 1 'emploi chez les courtiers en valeurs mobilières, les avocats, les comptables. Les simulations évaluent à 11531 l'emploi direct (en pers.-année), à 382 l'activité indirecte générée sur la maind'oeuvre, pour un total de 1535 pers.-annēe pour 1a pēriode 19801985. La masse salariale correspondante se chiffre à $25,1 \$$ millions directs, 9,0 \$ millions indirects, soit $34,1 \$$ millions. Les sommes versées en fiscal itē au gouvernement du Québec atteignent 6,8 millions, dont 2,2 \$ millions en parafiscalité, 3,9 \$ millions en impôts sur les salaires et 0,7 \$ millions en taxes indirectes. Du côté du fédéral, les paiements fiscaux totalisent 4,9 \$ millions, dont 2,6 \$ millions en impôts sur les salaires, 1,9 \$ million sur la parafiscalité et $372000 \$$ pour les taxes indirectes. Les gouvernements fēdéral et quēbécois rēcupèrent environ $22 \%$ des $53,9 \$$ millions de valeur ajoutée directe et indirecte générée 1 .

1. Précisons que ces estimés sont relativement conservateurs, compte tenu de l'emploi du sous-secteur des courtiers en valeurs mobilières pour évaluer l'impact sur les courtiers, les comptables et les avocats. Nous croyons que si les emplois totaux générés demeurent relativement comparables, la valeur ajoutēe engendrée (donc, les versements fiscaux) demeure plus importante pour ces deux dernières professions. 
Les achats découlant des frais d'émission et d'administration se concentrent quasi-exclusivement dans le domaine tertiaire: produits du papier, services têléphoniques, services récréatifs, publicité, postes. Le tableau $V$ nous en livre un aperçu plus qu'exhaustif. Ces données proviennent aussi des simulations effectuées au B.S.Q.

\subsubsection{3 découvertes}

L'étude d'impact économique recense 25 découvertes (gisement avec réserve) redevables aux actions accréditives, dont huit deviendront des mines en production à court terme. L'étude précise aussi que l'inventaire d'onces d'or en réserve dans le sous-sol a été "doublé", de même que la production du nombre d'onces d'or. 
TABLEAU $V$ : RETOMBEES ECONOMIQUES DES DEPENSES D'EXPLORATION

(EN MILLIER DE \$)

\section{1- EMPLOIS ET VALEUR AJOUTEE}

\begin{tabular}{|c|c|c|c|c|c|c|}
\hline & \\
\hline & \multicolumn{3}{|c|}{ F.E.C. } & \multicolumn{3}{|c|}{$\begin{array}{l}\text { FRAIS D'EMISSION } \\
\text { ET D'ADMINISTRATION }\end{array}$} \\
\hline EMPLOIS ET VALEUR AJOUTEE & DIRECTS & $\begin{array}{l}\text { INDIRECTS } \\
\text { EN AMONT }\end{array}$ & TOTAUX & DIRECTS & $\begin{array}{l}\text { INDIRECTS } \\
\text { EN AMONT }\end{array}$ & TOTAUX \\
\hline Main-d'oeuvre (pers.année) & 3205 & 1776 & 4981 & 1153 & 382 & 1535 \\
\hline Salaires et gages (avant impôts) & 97804 & 42231 & 140035 & 25091 & 8990 & 34081 \\
\hline Autres revenus bruts (avant impôts) & 33092 & 27396 & 60488 & 8344 & 8258 & 16602 \\
\hline Valeur ajoutēe (au coût des facteurs) & 130896 & 69606 & 200502 & 33441 & 17248 & 50689 \\
\hline
\end{tabular}

Source:

Calculé à partir de l'étude d'impact du B.S.Q., des sondages de Maheu Noiseux, des données d'Energie, mines et ressources Canada sur les financements horsQuēbec. 
TABLEAU VI : RETOMBEES ECONOMIQUES DES DEPENSES D'EXPLORATION

(EN MILLIER DE \$)

\section{2- PAIEMENTS FISCAUX}

$$
\text { F.E.C. FRAIS D'EMISSION }
$$

ET D'ADMINISTRATION

\begin{tabular}{|c|c|c|c|c|c|c|}
\hline \multirow[b]{2}{*}{ RECETTES FISCALES } & \multicolumn{3}{|c|}{ INDIRECTS } & \multicolumn{3}{|c|}{ INDIRECTS } \\
\hline & DIRECTS & EN AMONT & TOTAUX & DIRECTS & EN AMONT & TOTAUX \\
\hline Revenus du gouvernement du Québec & $27 \quad 721$ & 10785 & $38 \quad 506$ & $\underline{4885}$ & 1913 & 6798 \\
\hline $\begin{array}{l}\text { - Impôts sur salaires } \\
\text { - Parafiscalitē } \\
\text { - Taxes indirectes }\end{array}$ & $\begin{array}{rr}13 & 514 \\
7 & 797 \\
6 & 410\end{array}$ & $\begin{array}{ll}5 & 111 \\
3 & 443 \\
2 & 231\end{array}$ & $\begin{array}{rr}18 & 625 \\
11 & 240 \\
8 & 641\end{array}$ & $\begin{array}{ll}2 & 779 \\
1 & 589 \\
& 517\end{array}$ & $\begin{array}{l}1083 \\
625 \\
205\end{array}$ & $\begin{array}{ll}3 & 862 \\
2 & 214 \\
& 722\end{array}$ \\
\hline Revenus du gouvernement fédēral & 16134 & 6432 & $22 \quad 566$ & 3545 & 1309 & 4854 \\
\hline $\begin{array}{l}\text { - Impôts sur salaires } \\
\text { - Parafiscalité } \\
\text { - Taxes indirectes }\end{array}$ & $\begin{array}{ll}9 & 637 \\
4 & 310 \\
2 & 187\end{array}$ & $\begin{array}{ll}3 & 530 \\
2 & 101 \\
& 801\end{array}$ & $\begin{array}{rr}13 & 167 \\
6 & 411 \\
2 & 988\end{array}$ & $\begin{array}{ll}1 & 875 \\
1 & 417 \\
& 253\end{array}$ & $\begin{array}{l}754 \\
436 \\
119\end{array}$ & $\begin{array}{ll}2 & 629 \\
1 & 853 \\
& 372\end{array}$ \\
\hline $\begin{array}{l}\text { Recettes gouvernementales (Québec } \\
\text { et fédéral) }\end{array}$ & $43 \quad 855$ & $17 \quad 217$ & $61 \quad 072$ & 8430 & 3222 & 11652 \\
\hline
\end{tabular}




\subsubsection{Système de financement}

Il existe deux façons de financer une entreprise d'exploration, lesquelles peuvent être aussi applicables à toute entreprise: le placement privé et le placement public.

\subsubsection{1 placement privé}

Une sociēté qui veut émettre des titres (vendre des actions) doit le faire selon certaines règles.

Elle doit ainsi demander l'autorisation de la Commission des valeurs mobilières du Quëbec. Cette dernière s'assurera que 1 'information nécessaire est donnée aux épargnants.

Certaines entreprises sont dispensées de l'obligation d'établir un prospectus à 1 'occasion d'un placement $d^{+}$actions. Il s'agira souvent d'une entreprise familiale ou d'une société appartenant à des personnes qui ont des liens avec l'entreprise et qui n'ont pas fait publiquement appel à l'épargne. On dit alors qu'il s'agit d'une sociēté fermée car son financement ne provient pas du public.

Une sociēté est fermée au sens de la loi si ses documents constitutifs:

- limitent le nombre des actionnaires à 50 (sans tenir compte, toutefois, des actionnaires qui sont ou qui ont été à son emploi);

- interdisent toute forme de publicité en vue de la vente des actions;

- prévoient des restrictions quant aux modalités de vente des actions. 
Dès que l'un des critères précédents n'est pas respecté, la sociēté doit obtenir 1 'autorisation de la Commission.

La prudence recommande aux dirigeants de s'assurer auprès de la Commission qu'ils ont bien suivi l'application de cette partie de la loi avant de procéder à une émission.

Généralement, les titres sont vendus à escompte avant de procéder à une émission publique. Les actionnaires de fondation prennent un plus grand risque et, le placement ētant moins liquide, se voient offrir des chances de rendement supérieur grâce à 1 'escompte.

\subsubsection{2 le prospectus lors du placement public}

Une sociētē qui désire placer ses titres auprès du public doit établir un prospectus. Ce document est important. Il réunit des informations sur 1 'objet de l'émission, sur les objectifs de la société et sur sa santé financière. On y trouvera des ètats financiers importants à consulter. Le prospectus permet à l'acquéreur éventuel de titres de guider sa décision d'investissement en évaluant les risques par rapport aux bénéfices espérés. C'est donc un document qu'il faut lire avec soin. Si vous n'êtes pas tout à fait familier avec ce sujet, vous ne devez pas hésiter à demander les conseils d'un spécialiste.

Tout prospectus, avant d'être diffusé, doit être visē par la Commission. Celle-ci ne cautionne jamais l'émission comme telle; son visa $n$ 'est pas une recommandation. El le refusera cependant son visa si 7 'information présentēe dans le prospectus n'est pas complète ou que la protection des épargnants 1 'exige. 


\subsubsection{3 le prospectus provisoire}

Avant de placer des titres, une société peut vouloir sonder le marchē afin de vérifier si son émission trouvera preneur. Elle établit alors un prospectus provisoire qui peut omettre certaines informations du prospecus définitif, notamment celles concernant le prix et le nombre de titres à être placés.

Le courtier pourra donc demander à 1 'épargnant si l'émission 1 'intéresse, mais ne pourra accepter son engagement. Ce n'est que lorsque le prospectus définitif sera visé qu'il pourra le fairel.

Le prix du titre est établi entre T'entreprise émettrice et la maison de courtage. Les deux parties évaluent le marché, ce que 1 'épargnant est prêt à accepter comme risque et comme produit.

\subsubsection{Technologie}

\section{1 .11 .1 constat}

L'entreprise d'exploration procède généralement en deux étapes. La première étape consiste à établir une carte géologique générale pour que les compagnies d'exploration se choisissent des zones cibles. Au Québec, les services gouvernementaux sont très impliquēs dans cette activité.

La seconde étape est plus ponctuelle et relève en presque totalité de la responsabilité de l'entreprise privée. C'est lors de cette ètape que sont réalisés les levées gēologiques, géophysiques, géochimiques, etc.

\footnotetext{
1. Le marché des valeurs mobilières, Commission des valeurs mobil ières
} 


\subsubsection{2 firmes de consultation}

La consultation offre une diversité de levées, de mëthodologies, d'équipement dans les sciences physiques, chimiques, géologiques. Les firmes de consultation s'implantent facilement dans cette industrie à cause de l'importance de l'expertise et des besoins importants en capitaux pour se procurer les équipements.

\subsubsection{3 évolution de 1a technologie}

Des avances technologiques considérables en géophysique sont réalisées quotidiennement. L'entreprise de 1'exploration en est la grande gagnante. Le traitement informatique des données est chose courante. Des logiciels facilitent l'interprétation des levées, les techniques de présentation sont améliorées (cartes en couleur).

La géochimie, grâce à 1 'arrivée de l'informatique, devient un nouvel outil. Une sophistication des types de relevés voit le jour (humus, eau, lithogéochimie), le nombre d'éléments traités augmente grâce à 1 'utilisation de 7 'ordinateur.

De nouvelles techniques de forage voient le jour (forage à circulation renversée, forage sonic). Ces technologies ou méthodes nouvelles permettent l'obtention d'informations supplémentaires à moindre coût.

Dans la science de la géologie, de nouvelles spécialités sont apparues. On enseigne maintenant la géologie structurale dans les universités. Il s'agit d'une application raffinée de la science géol ogi que. 


\subsubsection{4 tendances}

L'utilisation de l'ordinateur fait maintenant partie du quotidien de la géophysique (au sol et aéroportée, et par satellite), de la géochimie (humus, roches) et de la gēologie. La spécialisation se retrouve au niveau de l'interprétaion ou de la mise à jour de nouvelles mêthodes. L'industrie de la consultation se caractérise par la sur-spécialisation. Des services de laboratoire, des centres de recherche sont accessibles à l'industrie (analyse par le feu, analyse par absorption atomique, les laboratoires se spécialisent sur la récupération, etc.).

\subsubsection{Physique}

\subsubsection{1 terrains miniers}

Une compagnie d'exploration qui désire s'adonner à ses activités doit possēder des propriétēs minières dans des camps miniers établis ou en voie de le devenir.

Un camp minier est d'abord un territoire géographique et géologique aussi. Il compte génēralement des mines en opération, une gēologie favorable et des indices de minēralisation. Lorsque les mines sont à découvrir, on dit alors qu'il s'agit d'un camp d'exploration.

\section{$2.1 .12 .2 \underline{1 \text { 'Abitibi }}$}

En Abitibi, nous retrouvons plusieurs camps miniers: Noranda, Val d'Or, Matagami, Joutel, Casa-Berardi. Ces camps miniers se retrouvent le long de ceintures volcano-sēdimentaires qui s'étendent sur plusieurs centaines de milles de la frontière de l'Ontario jusqu'à Chibougamau. Il y a un minimum de cing ceintures en AbitibiTémiscamingue et elles correspondent à des cassures régionales ou 
des dêplacements importants. La carte géologique suivante est plus explicite (annexe II).

2.1.12.3 la localisation

Donc, il est évident que la localisation des propriētés minières à I'intérieur de ces ceintures (20 à 40 milles de large et 200 à 400 milles de long) est primordiale. Certains endroits possèdent des caractéristiques plus favorables à la déposition de minéralisations le long de ces accidents régionaux géologiques et ce sont ces endroits que les compagnies d'exploration tentent de prendre pour cible et de possēder (annexe II).

\subsubsection{Concurrence}

\subsubsection{1 formes de concurrence}

Il existe de la concurrence pour deux objets entre les compagnies publiques juniors. La première est au niveau de l'argent dans le financement. La seconde est au niveau de la qualité des terrains.

\subsubsection{2 compëtition indirecte}

La compétition pour 1'argent se retrouve sous différentes formes. Il existe d'abord une compétition avec les autres vēhicules d'investissements offerts au public. Nous qualifions cette compétition d'indirecte. Le Régime d'épargne-actions du Quēbec est un fort compétiteur. Les formes d'investissements disponibles sont les dépôts à terme, les comptes d'épargne, les obligations, les obligations d'épargne, les fonds d'investissements, les actions ordinaires transigées en bourse et les actions de toutes catégories transigées en bourse. En fait, il existe un volume d'argent disponible et les vēhicules se le partagent. Il s'agit d'un marché 
compétitif où l'on utilise à outrance le marketing pour conquērir sa part de marché.

\subsubsection{3 compétition directe}

La compétition directe provient de toute compagnie minière offrant ses actions au public investisseur sur le marché primaire ou secondaire. Au Canada, il existe quatre bourses qui ont des inscriptions de compagnies minières (Montréal, Toronto, Alberta et Vancouver). Sur 1 a Bourse de Vancouver, nous retrouvons à peu près 1600 inscriptions de compagnies juniors. Sur la Bourse de Montréal, il y a à peu près 100 compagnies juniors d'inscrites.

Bien entendu, de plus en plus de compagnies voient le jour localement et dans la province. Il semble y avoir suffisamment de capitaux et de terrains pour de nombreuses compagnies. La différence entre vancouver et Montréât se trouve dans le montant des souscriptions. En fait, sur le marché de Vancouver, les appels à 1 'épargne publique sont plutôt modestes. Sur le marché de Montréal, les appels à l'épargne publique sont imposants ( 1 million de dollars et plus par souscription). Donc, il peut y avoir plus de compagnies pour se partager le montant d'argent, elles feront des souscriptions plus petites.

Nous retrouvons au plus une trentaine de compagnies établies en région. Il n'existe pas encore de forte compétition entre les "juniors établies régionalement". Il n'y a pas encore eu de compētition pour le capital. En fait, les investisseurs locaux investissent dans la junior qui a une propriété à proximité de la localité. A titre d'exemple, les investisseurs locaux de Val d'Or ont tendance à supporter les juniors de val d'or. Il en est de même pour Rouyn-Noranda. Cependant, une junior bien impliquée dans un secteur va s'attirer plus d'investissements de ce secteur. Citons 
T'exemple de Ressources Eider inc. qui a attiré fortement les investisseurs de Lebel-sur-Quévilion.

De nombreux promoteurs de Vancouver réalisent maintenant des appels à 1 'épargne publique au Québec à cause du climat fiscal favorable. La plupart d'entre eux ont un minimum de 10 à 20 années d'expérience. Plusieurs ont déjà connu des succès techniques impressionnants (découvertes). Donc, la ressource humaine (1'expertise technique) est un ēlëment de compëtition très important. Dans ce sens, la compétition de Vancouver est importante.

Il y a aussi la compétition des autres abris fiscaux. Au Québec, le régime d'ëpargne-actions a permis à plus de 125 compagnies de réaliser des appels à 1 'épargne publique en 1986. Le régime d'épargne-actions est très populaire et concurrence fortement les actions accréditives. Les investissements dans les films, dans les immeubles (articles 31 et 32 ) sont aussi des compétiteurs.

\subsubsection{4 compétition pour les terrains}

Comme nous l'avons expliqué dans les paragraphes sur l'environnement physique, à l'intérieur des ceintures favorables, $i 1$ existe des cibles privilégiées. Ces cibles privilégiées (terrains) s'acquièrent souvent à des prix élevés. Les compagnies "juniors" sont souvent en compétition pour 1 'acquisition des meilleurs terrains.

\subsection{LE SYSTEME ETUDIE}

\subsubsection{Organigrame}

L'organigramme met en évidence le processus décisionnel de Normétal inc. en tant qu'entreprise publique. 
2.2.1.1 l'assemblēe des actionnaires

L'entreprise doit cependant tenir une assemblée annuelle pour les actionnaires enregistrés auprès du fiduciaire. Le nombre d'actionnaires de Normétal Inc. atteint approximativement 400 individus.

2.2.1.2 le Conseil d'administration

Le Conseil d'administration était composé de six individus. Depuis octobre 1986, il est composé de cinq individus, à la suite de la démission de 1 'un de ses membres.

2.2.1.3 officiers

Les offiers de l'entreprise sont:

un président;

deux vice-présidents;

un secrētaire.

2.2.1.4 direction

Trois membres du Conseil d'administration, tout en étant officiers, composent la Direction:

un directeur gēnéral;

un contrôleur financier;

une directrice de 1 'exploration.

\subsubsection{Rôle du Conseil d'administration}

Le rôle du Conseil d'administration est surtout centré sur 1'approbation des dépenses importantes, des politiques et des stratégies d'entreprises: 
- approbation des projets d'exploration (budget, travaux, échéanciers);

- approbation des contrats (entrepreneurs et consultants) supérieurs à 15000 \$ (clauses) (qualité des firmes);

- approbation des politiques d'achat, de location (soumissions);

- approbation des acquisitions de propriétés, options et ententes conjointes;

- politique salariale de l'entreprise;

- préparation des plans de "stocks options". 
FIGURE II : ORGANIGRAMAE NORMETAL INC.

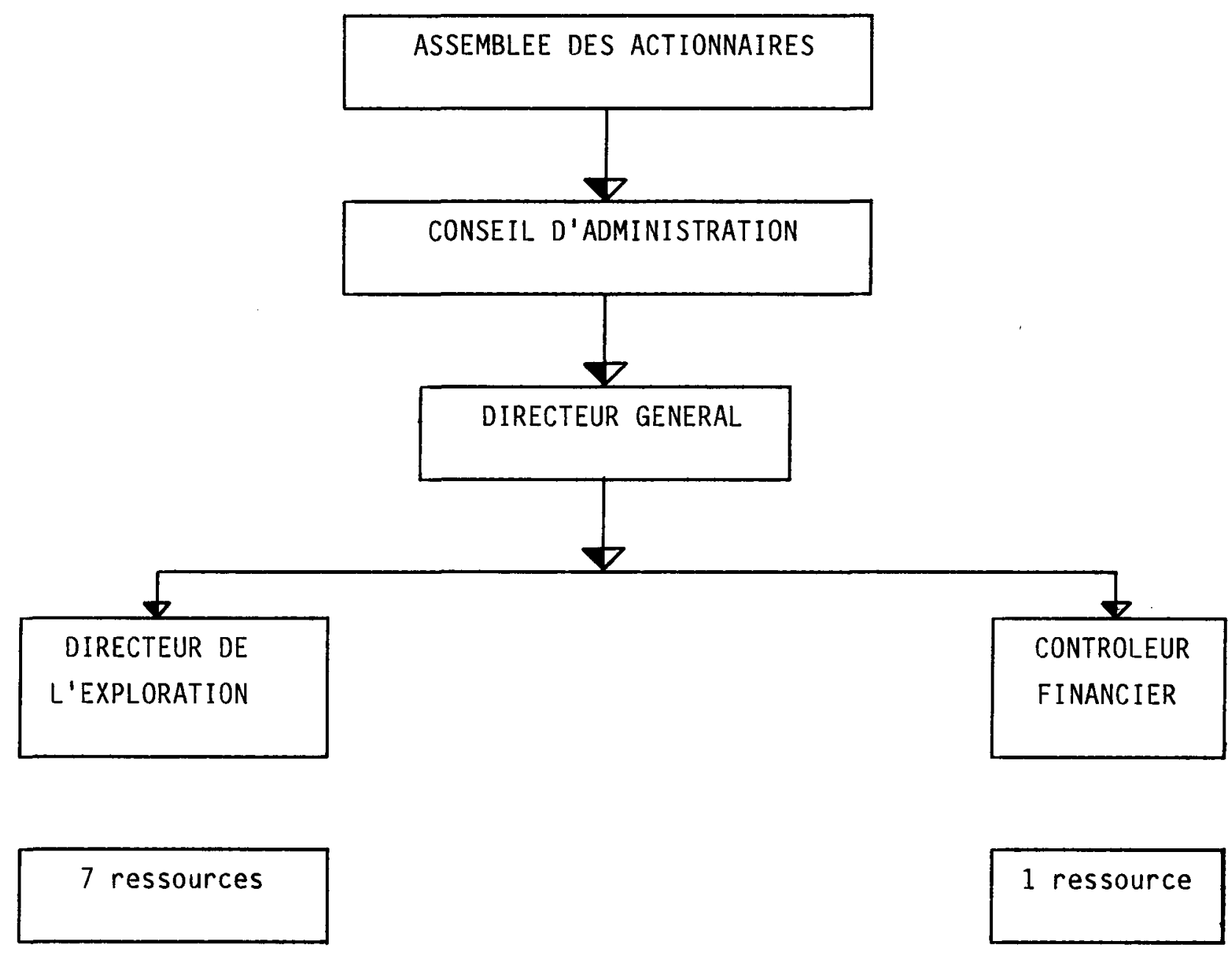

CONSULTANTS 


\subsubsection{Description des tâches}

L'équipe de Direction possède une liste des tâches formelle décrivant les responsabilités de chacun. Les autres employés de 1 'entreprise dans le service de l'exploration possèdent aussi une description des tâches.

\subsubsection{1 directeur général}

- Planification financière (financement).

- Coordination des services financiers et géologiques de l'entreprise.

- Application de la politique de relations publiques de l'entreprise.

- Autorisation des décisions financières non budgétées en-deça de $15000 \$$.

- Relations avec le Conseil d'administration et les actionnaires.

- Préparation des projets de développement de l'entreprise.

- Autorisation des mandats des consultants.

\subsubsection{2 responsable de l'exploration}

- Planification des travaux d'exploration (activités et budgets).

- Supervision des travaux du personnel d'exploration.

- Supervision des travaux des sous-contractants et des consultants (coupe de ligne, dēcapage, gēol ogie, gēophysique, forage).

- Levés géologiques, cartographie.

- Supervision et planification des forages.

- Conseirs et interprétations. 


\subsubsection{3 contrôleur financier}

- Tenue des registres comptables.

- Etats financiers mensuels, annuels.

- Gestion des titres miniers.

- Administration générale (paiements, comptabilité par projets, paies, pièces).

- Supervision du personnel administratif.

- Gestion financière et approbation des dépenses budgétaires endeça de $15000 \$$.

- Préparation des budgets.

\subsubsection{4 politiques opérationnelles}

L'entreprise possēde des politiques en matière de location et d'achat, d'achat d'actions et de questions salariales. Les politiques de location et d'achat àinsi que Tes politiques salariales sont approuvées par le Conseil d'administration. Les politiques d'achats d'actions et d'incitation à la découverte doivent être entérinées par la prochaine assemblée annuelle.

\subsubsection{5 règlements}

L'entreprise possède des lignes de conduite non ēcrites en matière d'horaire et de temps supplémentaire.

\subsubsection{6 aménagement}

Le siège social de 1 'entreprise est situé au 144 C, rue Principale, à Rouyn-Noranda. Il s'agit d'une location. L'entreprise possède deux bureaux d'exploration situés à Normétal et à Barraute. Ces bureaux sont des locations. 
L'entreprise possède aussi deux "core shack" extérieurs à proximité des bureaux d'exploration pour ranger les carottes de forage.

\subsection{LE PROCESSUS DE TRANSFORMATION}

\subsubsection{Description du processus}

Le processus de notre organisation comprend les activités de gestion et de transformation, interreliēes dans un flux d'argent, de personnel et de ressources physiques. Le processus décrit les opérations rēelles en séquence logique. Les activités essentielles de 1 'entreprise, celles qui font sa raison d'être, sont décrites. 
FIGURE III : PROCESSUS DE TRANSFORMATION DES ACTIVITES DE NORMETAL INC.

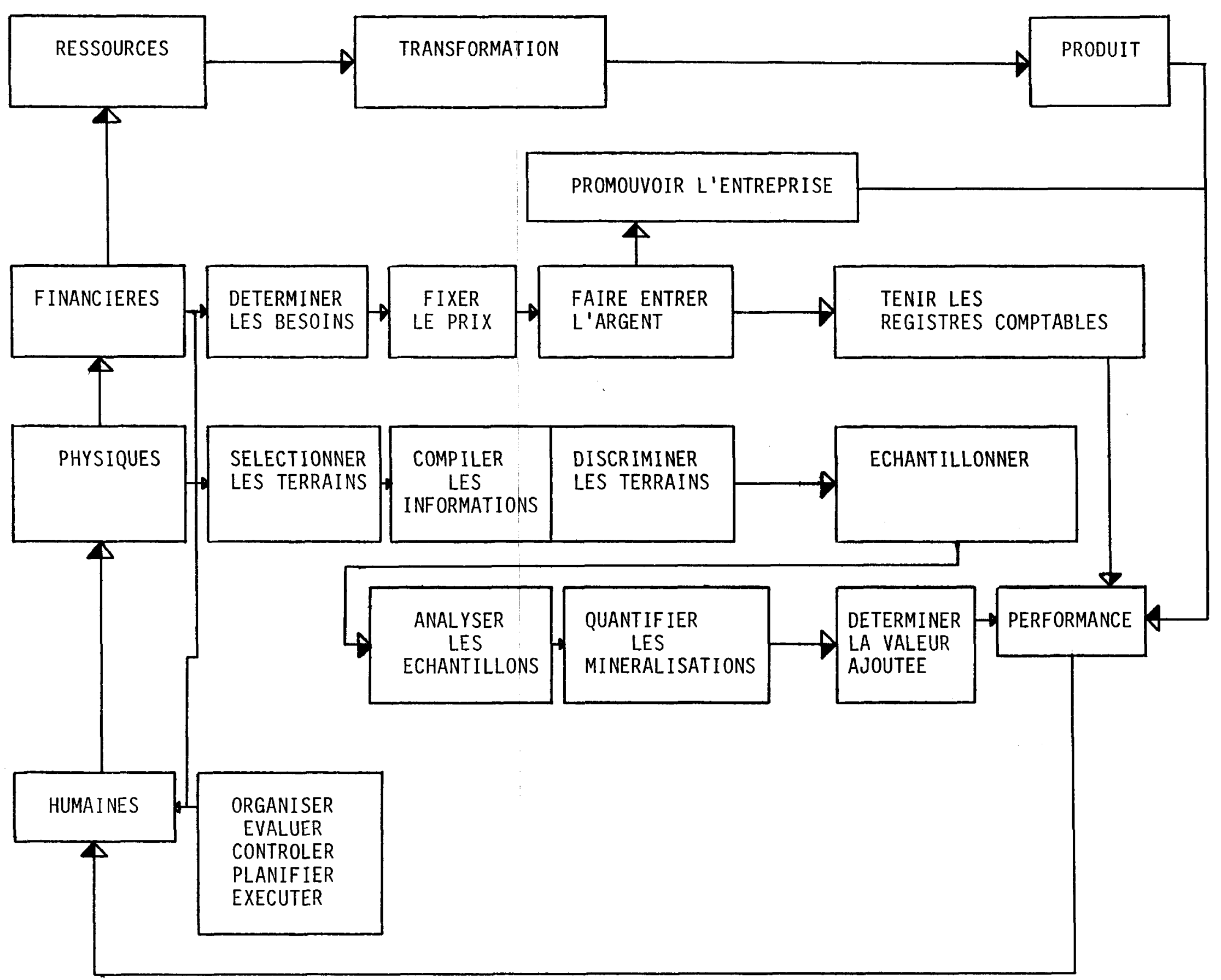




\subsubsection{Expl ication du processus}

Les opérations sont organisēes sur un diagramme sans faire rēférence au responsable de 1 'exécution.

Il est essentiel de définir ou d'expliquer le diagramme afin de saisir le processus de transformation.

FINANCIERES

DETERMINER LES

BESOINS FINANCIERS

FIXER LE PRIX

PROMOUVOIR

L'ENTREPRISE

FAIRE ENTRER

L'ARGENT

TENIR LES REGISTRES COMPTABLES
C'est l'ensemble des capitaux disponibles directement ou indirectement pour les activités de 1 'entreprise. Les capitaux sont fournis par les investisseurs ou d'autres entreprises, lesquels constituent les clients.

A partir des prévisions budgétaires pour les dépenses d'exploration et d'administration, on établit le niveau des dépenses.

A partir de la valeur comptable nette de l'entreprise, de la prime que l'investisseur est prêt à payer pour la valeur comptable en fonction du potentiel de 1 'entreprise, on fixe le prix de vente de l'action.

Faire connaitre au public 1 'entreprise pour susciter 1 'intérêt des investisseurs.

Choisir les canaux de distribution pour offrir les actions aux investisseurs éventuels.

Enregistrer les opērations comptables, les tenir à jour et les communiquer à l'actionnariat aux autorités régulatoires (Bourse de Montréal, Commission des 
valeurs mobilières du Québec) à intervalle rēgulier, ainsi qu'au public investisseur en général.

PHYSIQUES

C'est l'ensemble du portefeuille de propriétés minières que $T$ 'entreprise possède et pour lesquelles elle effectue (ou pas) des travaux d'exploration seule ou avec d'autres partenaires.

SELECTIONNER LES TERRAINS

COMPILER LES

INFORMATIONS

DISCRIMINER LES TERRAINS

ECHANTILLONNER

ANALYSER LES ECHANTILLONS
Parmi l'ensemble des terrains disponibles sur le marché ou offerts à l'entreprise, on choisit les plus favorables et on procède à 1 'acquisition.

C'est dresser un inventaire de toutes les informations ou travaux effectués ou recueillis par les prēcédents détenteurs en un seul endroit (carte) pour orienter les prochaines étapes de 1 'exploration.

Grâce à des relevēs gēophysiques, géochimiques et géologiques, à des méthodes techniques différentes selon les minēralisations recherchées, il s'agit d'ēliminer le maximum de territoire pour conserver la cible la plus petite possible et la plus favorable.

A partir de la cible la plus favorable déjà déterminée, il s'agit de recueillir par des méthodes de forage et autres méthodes le plus d'échantillons possible du sous-sol.

A partir des échantillons recueillis, on procède à 1 'analyse chimique ou autres afin de déterminer les substances minērales contenues dans 1 'échantillon. 
QUANTIFIER LES

MINERAL ISATIONS

DETERMINER LA VALEUR AJOUTEE

HUMAINES

EXECUTER

ORGANISER

EVALUER

CONTROLER

PLANIFIER
Lorsque 1 'ensemble des substances minérales sont connues de l'ensemble des ëchantillons, il s'agit de dresser une carte sur laquelle on tente de prouver la continuité des minéralisations ou de déterminer que les minéralisations rencontrées constituent un tout.

Lorsque les minéralisations rencontrées constituent un tout, il suffit de déterminer leur valeur économique en termes techniques (tonnage et teneur).

Ce sont les employés de l'entreprise (professionnels et techniciens), les consultants et les contracteurs qu'elle utilise.

C'est réaliser les tâches techniques par des flux physiques et monétaires (dessins, entrées comptables, évaluation financière, évaluation technique).

C'est l'organisation matērielle et temporelle de toutes les activités d'exploration et d'administration de 1 'entreprise.

C'est déterminer la qualité des propriētēs miniēres ainsi que les besoins financiers des projets de 1 'entreprise.

S'assurer de T'exécution et de la qualité des travaux effectués par les employés et par les consultants.

C'est décider des types de métaux recherchēs (donc, des propriétēs et de leur diversité gēographique). C'est aussi décider du niveau d'activités de 1 'entreprise. 
LE PRODUIT

\begin{abstract}
C'est la performance dans les résultats d'exploration, de même que le rendement de 1 'investisseur. Le produit est une participation à une découverte qui deviendra une mine et qui produira des revenus. Cet objectif n'est pas atteint souvent; donc, il existe un produit intermédiaire: la performance boursière.

I1 S'agit 1à de 1'appréciation du prix de l'action. Cette appréciation varie en fonction des quantités de minéralisation découvertes et qui sont la propriēté de T'entreprise sans que ce soit une mine en production.
\end{abstract}

\title{
2.4 LES SOUS-SYSTEMES DE L'ENTREPRISE
}

\subsubsection{Ressources humaines}

\subsubsection{1 composition}

L'entreprise compte dix ressources permanentes et deux ressources utilisées à temps partiel.

\section{Temps complet}

2 secrétaires

1 comptable

3 géol ogues

3 techniciens miniers

1 administrateur

\section{Temps partiel}

2 hommes de terrain 


\subsubsection{2 expérience}

Le comptable possède 20 annēes d'expérience, dont deux années auprès des compagnies d'exploration. Cependant, il suit l'évolution de $l$ 'exploration comme investisseur et prospecteur depuis deux ans.

L'administrateur possède 15 années d'expérience, dont une seule en exploration. Cependant, depuis 10 ans, il est impliqué en exploration comme investisseur et prospecteur à son compte.

La géologue (senior) a 8 années d'expérience comme consultante et salariée en exploration. Elle a travaillé pendant trois ans comme consultante à son compte. Elle a agi comme directrice de projet d'exploration penant deux saisons d'exploration.

Le deuxième géol ogue senior possède 8 années d'expérience. Il est spécialisé en géochimie.

Le géologue (junior) a 7 années d'expérience en exploration, dont quatre années comme géologue de mine en Colombie-Britannique.

Un des techniciens miniers a une bonne expérience en exploration, un autre a simplement une annēe d'expérience en dessin (il étudie actuellement au Collège de l'Abitibi-Témiscamingue pour terminer son D.E.C. en technique minière). Le troisième a une expërience pratique et une scolarité incomplète.

\subsubsection{3 les consultants}

La constante au niveau des ressources humaines est définitivement le manque d'expérience dans 1 'organisation et la conduite des compagnies d'exploration. Tant au niveau de l'exploration que de l'administration, personne ne possède réellement le métier et un 
bagage d'expérience significatif, 1 'expērience étant cependant connexe à celle requise.

Au point de vue de l'exploration, la géologue senior connaît bien le réseau de consultants et $y$ entretient de bonnes relations. Sa connaissance des terrains miniers disponibles est intēressante, mais un peu limitée. Elle n'a pas encore connu de succès technique important, soit une dēcouverte.

Le géologue junior doit tout apprendre de la géologie de la région. Le second gēologue senior connaît assez bien les roches de I'Abitibi.

Pour les techniciens miniers, l'un est très peu porté vers le travail de terrain. Les deux autres sont assez flexibles; ils peuvent travailler autant sur le terrain que sur le dessin.

Le contrôleur financier a une expérience pertinente de 18 mois seulement, mais plus de 15 annēes d'expérience connexe.

Rien de particulier ne caractērise les employés à temps partiel, si ce n'est que 1 'un de ces employés est un prospecteur avec plus de 40 ans d'expérience.

Les consultants que 1 'entreprise utilise appartiennent au réseau de connaissance de la géologue senior. En géophysique, l'une des firmes est nouvelle et possède une excellente réputation, malgré son expérience limitée. Une autre firme est reconnue à travers le Canada pour la qualité de ses relèves et l'exclusivité de sa méthodol ogie.

En géologie, l'entreprise utilise une firme locale récemment formée. Il s'agit d'un groupe de professionnels des sciences de la terre qui 
a quittē une grosse firme internationale de consultation. Leurs services sont de qualité.

\subsubsection{4 propriētaires-dirigeants}

Le comptable et l'administrateur sont les deux gros actionnaires de 1 'entreprise. La participation du géologue senior au capitalactions est aussi importante.

Le constat sur les ressources humaines est à 1 'effet que les dirigeants n'ont pas un passé qui leur garantit le succès. Cependant, leurs niveaux respectifs d'éducation et la diversité de leur formation sont des atouts importants. Leur motivation et leur entrepreneurship sont évidents et connus dans le milieu de 1 'exploration.

Cet état du propriētaire-dirigeant peut poser des problèmes dans le processus décisionnel pour aborder la problématique des ressources humaines.

\subsubsection{Finances}

\subsubsection{1 états financiers}

Les états financiers de l'entreprise sont peu révēlateurs de la problématique de base de l'exploration. Ces mêmes états, au 31 novembre 1986, indiquent que Normétal possède approximativement $100000 \$$ en liquidité et placement depuis l'existence de l'entreprise. L'état de l'évolution de la situation financière indique que les fonds destinés à 1 'exploration sont épuisés. 
Le dernier rapport trimestriel révèle que l'entreprise possède $200000 \$$ en liquidité et placements à terme. Ces liquidités constituent son fonds de roulement.

\subsubsection{2 dilution}

Comme T'entreprise n'a pas de revenus de production, elle doit financer ses opérations futures par la vente de nouveau capitalactions. Ceci signifie que les actionnaires actuels acceptent de diminuer leur état de richesse grâce à 1 'arrivée de nouveaux actionnaires.

En fait, la richesse de l'entreprise se partage avec plus d'actionnaires. Il s'agit donc d'un processus d'appauvrissement. S'il s'agissait des mêmes investisseurs qui réinvestissaient, le résultat serait le même, s'il n'y a pas croissance des actifs.

Exploration minière La Sarre inc. détient les intérêts suivants dans chacune des propriétés: intērêt indivis de 50\% dans la propriété Barraute, intērêt indivis de $50 \%$ dans la propriété La Sarre et intērêt de $25 \%$ dans la propriēté Normétal.

Ces intérêts furent acquis de Exploration miniēre Normétal inc. par contrat de vente et d'entreprise en coparticipation, daté du 16 février 1987 et modifié le 4 juin 1987 pour les considérations suivantes:

- paiement de la somme de 75000 \$à Normétal à la signature de 1 'entente;

- émission de 750000 actions ordinaires bloquées de la Société en faveur de Normétal;

- travaux d'exploration sur les propriétés répartis ainsi: 
Propriēté Barraute:

- $400000 \$$ avant le 31 décembre 1987;

- 300000 \$avant le 31 décembre 1988;

- $300000 \$$ avant le 31 décembre 1989;

Propriété La Sarre:

- 500000 \$ avant le 31 décembre 1988;

Propriēté Normétal :

- 600000 \$ avant le 31 décembre 1987;

- 600000 \$ avant le 31 décembre 1988;

- $600000 \$$ avant le 31 décembre 1989.

Cet état de choses a pour effet de diminuer les besoins en capitaux de Normétal inc. et, par le fait même, la dilution de son capital.

\subsubsection{3 ententes}

Normētal inc. vient de signer une entente avec la Société d'exploration minière de La Sarre (filiale de Normétal) pour vendre un intērêt indivis dans ses propriētēs. La vente s'est effectuée pour des considérations financières, des actions de La Sarre inc. et des dépenses d'exploration.

Normétal inc. a aussi signé une entente de coparticipation avec Doelcam inc. pour l'évaluation du résidu minier. L'entente stipule l'obligation de Doelcam inc. de produire une étude de faisabilité. Si le résultat de l'étude est positif, la mise sur pied d'une entreprise de récupération sera effectuée conjointement. Les coûts et les revenus seront partagés également. 


\subsubsection{4 avenir financier}

La décision de vendre un intérêt dans les propriētés plutôt que d'émettre de nouvelles actions aura des conséquences importantes sur le plan financier. En 1 iquidité, Normétal inc. a approximativement 225000 \$ et en placement, à peu près 375000 \$ (actions de La Sarre). Du placement dans La Sarre, Normétal inc. pourra liquider $20 \%$ à n'importe quel moment, $10 \%$ par année pour les trois prochaines années et le solde, soit 50\%, 5'il y a une découverte ou une décision de production.

A court terme, l'entreprise est liquide et peut utiliser ses 1iquidités pour saisir toute opportunité.

A moyen terme, elle se retrouvera avec le problème du fonds de roulement déficitaire si elle n'a pas de revenus de production de ses projets ou d'autres projets. Sur un horizon de 24 mois, cela signifie qu'elle pourrait être dans l'obligation d'émettre de nouvelles actions pour obtenir des capitaux.

Quant à la possibilité d'avoir des revenus de production, Normétal inc. jouit d'un avantage en comparaison avec d'autres juniors. L'entreprise vient de signer une lettre d'entente avec une autre entreprise pour réaliser une étude de faisabilité complète sur son projet de résidu minier. Le partenaire doit déposer son étude pour décembre 1987. Si les résultats de l'étude sont positifs, une décision de production sera alors prise. Normétal inc. a aussi commandē une étude de préfaisabilité sur un gîte de zinc (306 800 t) qu'elle possède. Bien que ce dēpôt soit marginal, il est possible que 1'utilisation des méthodes nouvelles de minage et de financement puisse rendre sa récupération rentable. Normétal inc. a déjà rencontré des partenaires intéressés à ce type dé projet, mais n'a rien signé encore. 
La mise sur pied de ce projet est cependant complexe. Sa réalisation peut être retardée par de nombreux éléments: prix du zinc, acheteur du produit, accessibilité au financement.

\subsubsection{Technologie}

\subsubsection{1 gestion de T'entreprise}

Pour le moment, l'entreprise n'utilise pas une technologie bien particulière pour sa gestion. Ainsi, les opérations comptables, de dessin et de gestion de titres miniers sont effectuées sans support informatique. L'entreprise a cependant contacté les entreprises spécialisées dans le domaine pour obtenir ces services de gestion informatisēe en matière de titres miniers, de dessin et de comptabilité.

\subsubsection{2 gestion de 1'exploration}

Par le biais des firmes de consultation, l'entreprise, dans la dernière année, a fait appel à plusieurs méthodes et techniques avancées de prospection. Elle a utilisé les méthodes gēophysiques et géochimiques de prospection. Par les méthodes géophysiques, elle a eu recours à cinq techniques ou méthodes différentes, lesquelles nécessitent toutes un traitement informatique pour l'interprétation des donnēes. 


\subsubsection{Le marketing}

\subsubsection{1 prérequis pour une junior}

Le 24 janvier 1986, le journal North american gold mining industry news titrait: "People, property and promotion are keys to juniors mining success". L'auteur de l'article, par le seul titre qu'il lui a donné, présume parfaitement de l'essence de l'industrie de 1 'exploration.

La compagnie doit posséder un terrain avec du potentiel. Le terrain doit être localisé dans un camp minier reconnu ou en voie de le devenir. Les dirigeants de la compagnie doivent posséder l'expertise technique pour découvrir un dépôt minéralisē. Enfin, ils doivent être capables de "promouvoir" la compagnie pour faciliter 1 'accès aux capitaux. En fait, "the story must be told and sold".

\subsubsection{2 le communiqué de presse comme outil de promotion}

L'outil privilégié par les compagnies d'exploration est le "press release". Les principaux sujets traités dans les communiqués de presse sont en relation avec les activités de la compagnie. Enumērons quel ques-uns de ces sujets: acquisition de propriētés, activitēs de financement, valeur des minéralisations dans un dépôt, résultat de programmes d'échantillonnage et de forage, etc.

Les nouvelles sont généralement communiquées aux investisseurs en termes techniques. L'interprétation des communiqués est essentielle. D'ailleurs, les profanes sont souvent attirés par le sensationnalisme de la nouvelle.

Pour bien saisir ce phénomène, il est essentiel de donner quel ques exemples et de les interprēter. Une compagnie qui rapporte l'exis- 
tence de réserves nouvelles sur une propriété, sans préciser la nature des réserves, ne divulgue pas toute l'information à l'investisseur. En effet, il existe plusieurs catégories de réserves. Leur qualification est importante, car elle indique le degré de certitude de leur existence. Plus les réserves sont prouvées, moins grand est le risque.

De même, la diffusion des résultats de forage, en utilisant des valeurs non coupées, est une information moins précise. La pratique de l'industrie veut que les calculs de réserves se fassent avec des valeurs coupées. Ainsi, les très hautes valeurs aurifères ne peuvent hausser la moyenne. Sur un petit nombre de résultats, la conséquence est très significative.

La diffusion de la valeur brute d'un dépôt est impressionnante, mais elle ne permet pas à l'investisseur de connaître les coûts d'exploitation. Il est possible que le gisement ne soit pas économique et qu'il ne soit jamais mis en exploitation. Il en est de même si l'on ne connaît pas les taux de récupération et de dilution du minerai. En effet, lors du minage et du traitement du minerai, il y a des pertes qui diminuent la valeur du gisement.

\subsubsection{3 les propriétés comme base de promotion}

Donc, grâce au communiqué de presse, la compagnie junior rend compte de ses activités. C'est son moyen de vente. Le niveau de la junior reflète le contenu du communiqué. Il est faux de croire que la promotion est uniquement le sensationnalisme de la nouvelle. En fait, "peu importe 1 'emballage, il doit $y$ avoir quelque chose dans la boîte". Cette expression revient à dire que plus le niveau de 1 a junior est élevé, plus les communiqués peuvent être vendeurs ou de nature à intéresser les investisseurs. Donc, les propriétés sont à l'origine des activités. Elles constituent la base de la 
promotion. Le niveau d'avancement des projets est important (voir le tableau III).

\subsubsection{4 le produit}

Le produit n'est évidemment pas l'action ou le certificat d'actions. Dans la première partie du travail, nous avons défini le produit par l'existence de trois objectifs plus ou moins probables que nous avons illustrēs selon une courbe (tableau VII). Le produit, c'est une participation éventuelle dans une mine, une appréciation du prix de l'action, une économie d'impôt. En termes financiers, c'est une forme d'investissement, dont le taux de risque est réduit par l'abri fiscal dont la valeur est fluctuante.

L'objectif ultime pour les acteurs est "une participation à une découverte, laquelle deviendra un actif qui produira des revenus, soit une mine".. Cet objectif ultime n'est pas atteint souvent statistiquement. Donc, 1 'industrie a des objectifs plus rapprochés. Une appréciation du prix de l'action constitue un résultat "plus probable et plus rapproché", c'est aussi le produit.

Depuis quel ques annēes, l'industrie de l'exploration est considérée comme un abri fiscal. Le traitement fiscal accordé aux investisseurs permet aux acteurs d'avoir un objectif plus immédiat soit la récupēration d'impôt. Nous dressons une courbe de probabilité statistique pour démontrer la complexité du produit.

Il faut le constater: le produit a évolué depuis le début des années 1980 avec 1 'arrivēe des actions accréditives. Il existe trois besoins qui sont satisfaits par le produit. Cette distinction du produit en trois formes est très importante sur le plan stratégique. 


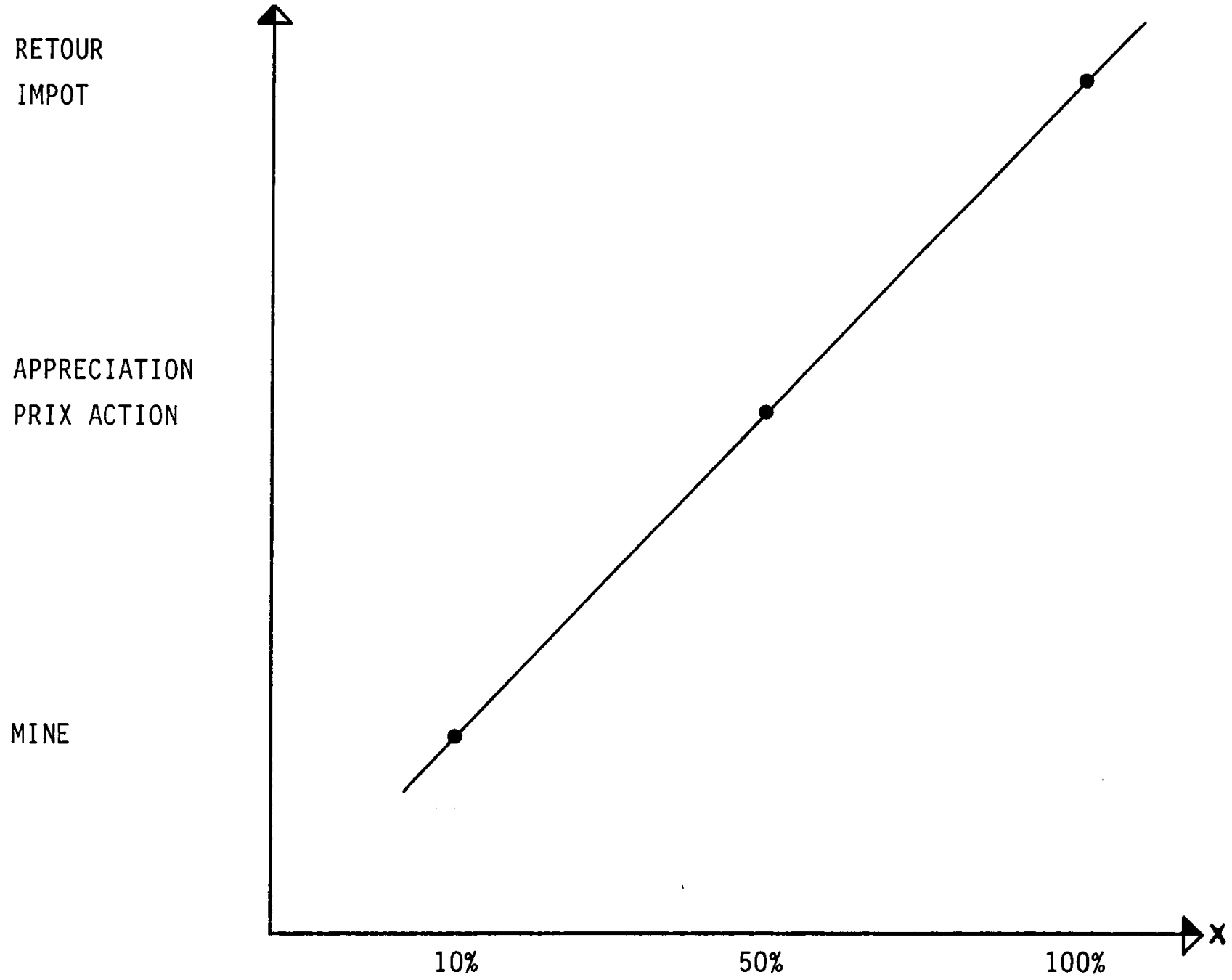

Axe des $X:$ PROBABILITE D'OCCURRENCE

Axe des Y: LES 3 DIFFERENCIATIONS DU PRODUIT

Cette courbe ne tient pas compte des facteurs temporels. De plus, les valeurs sur l'axe des $X$ sont hypothētiques et sont basées sur des connaissances empiriques. 
2.4.4.5 les différenciations du produit

Le produit, c'est un financement par des actions accréditives ou des sociētēs en commandite. Le taux d'abri fiscal varie d'une forme à 1 'autre. Certaines émissions offrent des bons de souscription en prime pour abaisser le coūt de l'investissement ou pour faciliter des financements futurs. Certaines émissions offrent aussi l'abri fiscal du R.E.A.Q. Le tableau I résume les différentes possibilités. Quelques exemples de modes de financement sont énumérēs, ainsi que leurs principales caractéristiques.

\subsubsection{6 réseau de distribution}

Un retour sur les concepts de marché primaire et secondaire nous apparaît ici important. Au Quëbec, il existe à peu près une dizaine de maisons de courtage qui font des émissions de compagnies minières dans le marché primaire. Les "super-maisons" de courtage avec des réseaux de succursales à travers le Quēbec (Lévesque et Beaubien, McLeod Young and Weir, Geoffrion Leclerc) ne font que des émissions très importantes. Il existe deux types de marchē que doivent satisfaire les compagnies "Junior": le marché primaire et le marché secondaire.

\subsubsection{1a différenciation des anal ystes}

Sur le marché secondaire, la différenciation est faite par les analystes des maisons de courtage. La qualité du produit est analysée par des experts. De nombreux facteurs servent à l'analyse: fonds de roulement, nombre d'actions en circulation, réserves, etc. Des ratios facilitent 7 'analyse. Il en résulte des recommandations qui sont diffusées à la presse financière, aux réseaux de courtage. Ces recommandations servent de base aux investisseurs pour rēaliser des investissements sur le marché secondaire. Les analystes tentent 
ainsi de dénicher l'action "sous-évaluée et avec le meilleur potentiel d'appréciation du prix de l'action".

C'est sur le marché secondaire que l'on reconnaît le plus l'influence du prix des métaux. Les analystes sont très influencēs par une hausse ou une baisse éventuelle du prix des métaux. De là toute 1 'importance de la planification stratégique dans l'acquisition ou le choix des propriétēs. Aujourd'hui, une junior a besoin d'un portefeuillle de propriétés basé sur l'or. Il n'y a cependant rien qui indique qu'une junior n'aura pas besoin de propriētés de métaux de base dans l'avenir. Nous considérerons le cyclisme des métaux lorsque nous discuterons de la planification stratēgique.

\subsubsection{8 la concurrence}

Nous l'avons déjà prēcisé, la compétition se retrouve sous différentes formes. Il existe une compétition avec les autres vēhicules d'investissements offerts au public. Nous qualifions cette compétition d'indirecte. Les formes d'investissements disponibles sont les dépôts à terme, les comptes d'épargne, les obligations, les obligations d'épargne, les fonds d'investissements, les actions ordinaires transigées en bourse et les actions de toutes catégories transigées en bourse. En fait, i1 existe un volume d'argent disponible et les véhicules se le partagent. Il s'agit d'un marché compétitif où les intervenants utilisent à outrance le marketing pour conquērir leur part de marchē.

La compétition directe provient de toute compagnie minière offrant ses actions au public investisseur sur le marché primaire ou secondaire. Au Canada, il existe quatre bourses qui ont des inscriptions de compagnies minières (Montréal, Toronto, Alberta et Vancouver). Sur la Bourse de Vancouver, nous retrouvons à peu près 
1600 inscriptions de compagnies juniors. Sur la Bourse de Montréal, il y a à peu près 100 compagnies juniors d'inscrites.

Bien entendu, de plus en plus de compagnies voient le jour localement et dans la province. Il semble y avoir suffisamment de capitaux et de terrains pour de nombreuses compagnies. La différence entre Vancouver et Montréal se trouve dans le montant des souscriptions effectuées par les compagnies inscrites. En fait, sur le marché de Vancouver, les appels à 1 'épargne publique sont plutôt modestes. Sur le marché de Montréal, les appels à l'épargne publique sont imposants ( 1 million de dollars et plus par souscription). Donc, il peut y avoir plus de compagnies pour se partager le montant d'argent; elles feront des souscriptions plus petites.

Nous retrouvons au plus une trentaine de compagnies établies en région. Il n'existe pas encore de forte compétition entre les "juniors établies régionalement". Il n'y a pas encore eu de compétition pour le capital. En fait, les investisseurs locaux investissent dans 1 a junior qui a une propriétē à proximité de la localité et aussi en raison de la connaissance des dirigeants des juniors.

De nombreux promoteurs de Vancouver rēalisent maintenant des appels à 7 'épargne publique au Québec à cause du climat fiscal favorable. La plupart d'entre eux ont un minimum de 10 à 20 années d'expérience. Plusieurs ont déjà connu des succès techniques impressionnants (découvertes). Donc, la ressource humaine (1'expertise technique) est un élëment de compétition très important. Dans ce sens, la compētition de Vancouver est importante.

Il y a aussi la compétition des autres abris fiscaux. Au Québec, le régime d'épargne-actions a permis à plus de 125 compagnies de 
réaliser des appels à 1'épargne publique en 1986. Le régime d'épargne-actions est très populaire et concurrence fortement les actions accréditives. Les investissements dans les films, dans les immeubles (articles 31 et 32 ) sont aussi des compétiteurs.

\subsubsection{9 publicité et advertising}

L'industrie de l'exploration a très peu recours à l'advertising. Quelques sollicitations sont effectuēes dans les journaux. Le prospectus lors d'une émission primaire, est probablement la plus importante dẻpense d'advertising. Il existe à cause d'une exigence légale. Il est souvent volumineux et pas très intéressant.

Occasionnellement, les entreprises vont se payer des publi-reportages. Elles vont aussi publier des cartes de localisation de propriētēs et des cartes géologiques qu'elles vont distribuer aux acteurs. Elles vont aussi assister à des conventions, des journées consacrées à 1 'exploration en organisant des kiosques.

L'industrie de l'exploration utilise la publicité de deux façons. La première forme de publicité vient de la presse financière et spécialisēe. A partir des communiqués de presse, des rencontres, des reportages, les journaux publient des articles sur les compagnies et leurs projets. Les compagnies juniors ne possèdent que très rarement un matériel publicitaire disponible pour les acteurs de l'industrie; ce n'est pas encore un réflexe d'avoir un bon système de relations publiques.

L'autre façon d'utiliser 1 a publicité provient de 1 a "rumeur". En fait, il s'agit d'un milieu avide de rumeurs. Il existe une tradition quant à l'utilisation de la rumeur; plus c'est secret, mieux c'est. Combien de fois une rumeur a pour origine le scellé imposē sur les boîtes de carottes de forage? Combien de courtiers 
et d'investisseurs cueillent leur information auprès du personnel des compagnies de forage?

En fait, il est relativement facile de monter une "opération publicitaire" grâce à la rumeur. C'est d'ailleurs à cause de cela qu'on associe souvent "l'exploration minière à la promotion plus ou moins honnête". D'ailleurs, les journalistes utilisent le terme "rumeur" tant que les résultats ne sont pas confirmés par une source officielle de la compagnie.

Peter Hunt, dans un article du journal The Northern Miner, publiē en juin 1985, titrait: "Confidence and trust are the essence of good public relation". Son article fait le point sur cette problématique de la confiance. Nous sommes en accord avec sa position. La compagnie doit être très responsable dans l'utilisation de la publicité et elle devrait utiliser un advertising professionnel pour établir la base de ses relations publiques.

\subsubsection{0 la complexité de l'industrie}

Il est bien évident que la promotion des juniors est une question complexe. Dans le constat, nous avons dégagé des éléments concernant la conceptualisation de la promotion. Conclure que la promotion d'une compagnie junior d'exploration se fait grâce à la communication à travers les communiqués de presse est un postulat inexact.

\subsubsection{1 le mix marketing et la décision stratégique}

En fonction du niveau de la compagnie junior (tableau III), ou plutôt du produit qu'elle représente, ell le peut choisir différentes alternatives. Ainsi, certains produits s'écoulent à travers des réseaux différents et à des prix différents. Il n'existe pas de 
règle précise, ou plutôt il en existe une fondamentale. Pour illustrer cette règle, il est possible de faire une analogie avec l'industrie du vêtement. Pour paraître bien habillé, un individu doit choisir un costume selon son point, une coupe selon son physique et, de préférence, à la dernière mode, si cela lui va bien. En bref, une junior avec un terrain "grass root" ne peut se vendre à un prix élevé par une grosse maison de courtage. C'est comme dans l'industrie de l'automobile, il existe des petites autos et des grosses autos luxueuses pour satisfaire tous les marchés.

\subsubsection{2 utilité du marketing}

Victor Buell, dans son volume intitulé: Marketing management a strategic planning approach, présente le marketing comme un système. Il parle de système à cause des interactions entre les environnements internes et externes.

Dans l'environnement interne, la compagnie exerce un contrôle sur le "Mix-marketing". L'environnement externe est moins contrōlable, mais la connaissance de variables incontrôlables de l'environnement externe peut permettre à la junior d'ajuster son "mix-marketing" (prix, produit, distribution, promotion).

L'industrie de l'exploration est complexe. L'environnement de la junior recèle tellement d'interactions et d'éléments incontrôlables (prix des métaux, mobilitē du capital, programmes gouvernementaux d'abris fiscaux, etc.) qu'elle doit s'efforcer de maitriser tout ce qui dépend d'elle, soit son "marketing".

Les concepts usuels du marketing (distribution, prix, produit, publicité, etc.) s'appliquent à l'industrie de l'exploration et peuvent facilement être utilisés. Cette industrie n'est pas diffërente des autres. 


\subsubsection{3 management marketing}

La junior doit élaborer un plan de marketing et l'exécuter. Cela signifie l'établissement d'un objectif, prévoir les ressources pour atteindre cet objectif. Tout ceci doit permettre la satisfaction des acteurs et des actionnaires. C'est de cette façon que la compagnie va se développer.

\subsubsection{Les actifs}

Les actifs de l'entreprise sont des liquidités, des placements et des terrains miniers à différents stades d'avancement. Le processus de transformation à la page 68 indique bien que les actifs de base de l'entreprise sont les terrains miniers. Ces terrains sont sa principale ressource physique.

Comme l'ētape ultime est de quantifier des minéralisations sur les terrains miniers, 11 est évident qu'un portefeuille de propriétés variè pose un problème d'évaluation des actifs miniers. Nous pouvons cependant comprendre que les terrains avec des minéralisations quantifiēes risquent d'avoir une valeur accrue, car ils permettent à 1 'entreprise de se concentrer sur des niveaux de projets plus avancés, tels que présentés dans le tableau VII.

\subsubsection{1. les propriētēs minières}

\subsubsection{1 titres et propriētés}

L'intērêt de Normétal inc. dans tous les terrains est majoritaire et ceux-ci sont entièrement payēs, à 1 'exception d'un récent ajout à la propriété Normétal, Barraute et Dal quier. 
NOM DE LA

PROPRIETE
NATURE DU TITRE ET TYPE DE PROPRIETE
POURCENTAGE

D' INTERET
RECHERCHE

METAUX

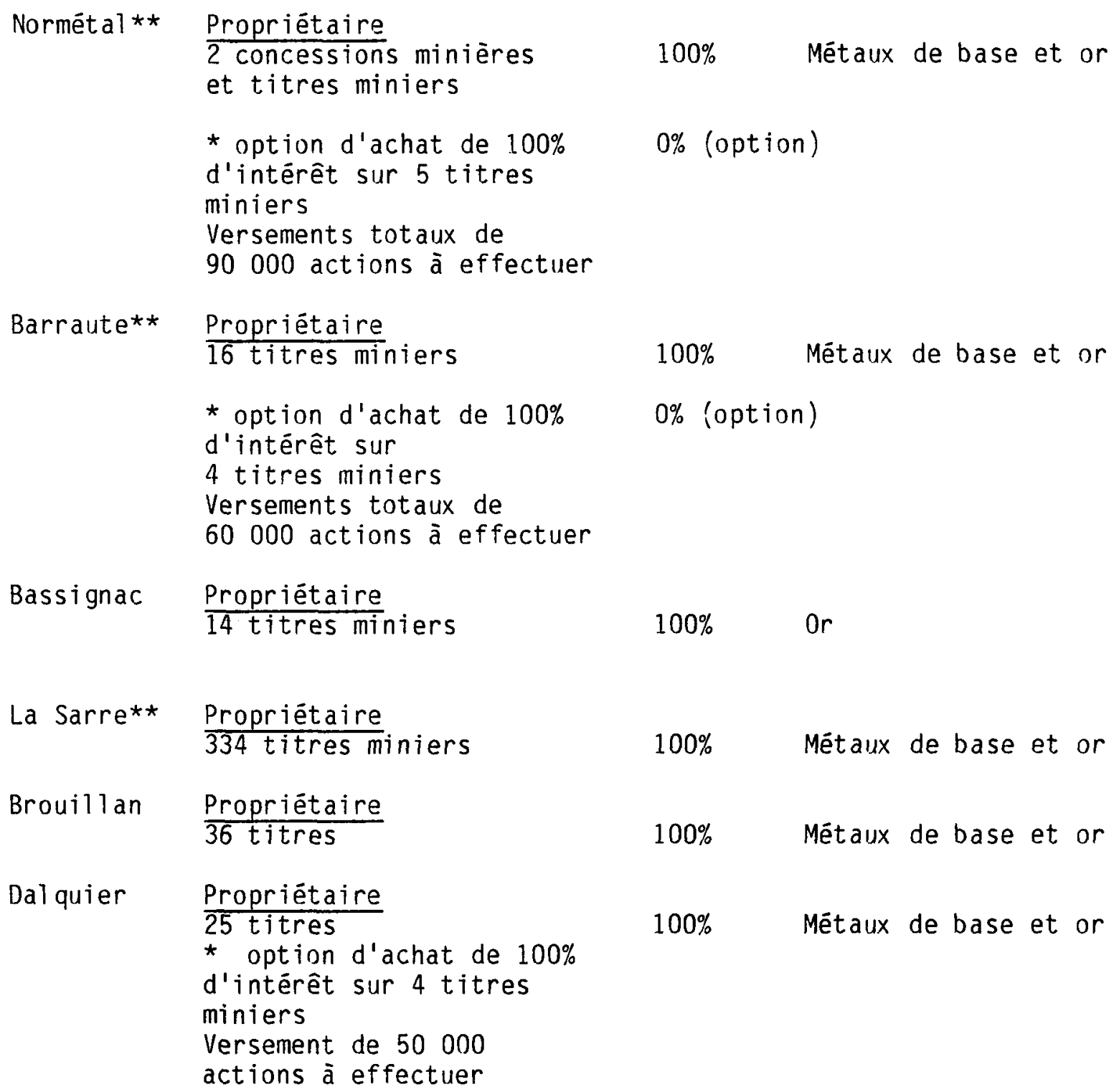

Bassignac Propriétaire

14 titres miniers

$100 \%$

Or

La Sarre** Propriétaire

334 titres miniers

$100 \%$ Métaux de base et or

Brouillan Propriétaire

36 titres

$100 \%$ Métaux de base et or

Dalquier Propriétaire

25 titres

* option d'achat de $100 \%$

$0 \%$ (option)

$0 \%$ (option)

$100 \% \quad$ Métaux de base et or

d'intērêt sur 4 titres

miniers

Versement de 50000

actions à effectuer

$100 \%$ Métaux de base et or

* Option: droit exclusif de devenir propriétaire moyennant certaines considērations monétaires et autres échelonnées sur un certain nombre d'années ou mois.

** : dans ces propriētēs, Exploration La Sarre inc. peut gagner un intérêt en contrepartie de travaux d'exploration. L'entente est décrite aux pages 77 et 78 . 


\subsubsection{2 obligations financières envers les propriétés}

En cas de production commerciale, certains de ces terrains sont assujettis à une royauté envers les anciens détenteurs:

Normétal :

royauté de $2 \%$ du produit net d'affinage. Normétal vient de racheter $2 \%$ de cette royauté qui était à 1 'origine de $4 \%$ pour une somme totale de $25000 \$$. Sur les terrains en option, 1 a royauté est de $3 \%$ du produit net d'affinage;

Barraute : royauté variant de $1 \%$ à $2 \%$ du produit net d'affinage et ce, sur huit titres;

Bassignac: $\quad$ aucune royauté;

La Sarre : royauté de $3 \%$ du produit net d'affinage;

Brouillan: aucune royauté;

Datquier : $\quad$ royauté de $2 \%$ du produit net d'affinage.

L'industrie considère le paiement d'une royauté de l'ordre de $3 \%$ à $4 \%$ du produit net d'affinage comme acceptable. Donc, toute royauté inférieure à cette règle de pouce est facile à honorer.

Normétal inc. peut en tout temps mettre fin aux options d'achat. Les options d'achat furent signées sur les propriétés de Normétal et Barraute pour des raisons stratégiques, soit l'agrandissement des propriétés pour la période durant laquelle Normétal Inc. explore de façon intensive ses terrains détenus à $100 \%$. Si l'entreprise décidait de se rendre jusqu'à la fin de l'option d'achat, ceci signifierait des déboursés de l'ordre de 150000 \$ comptant et en actions. Normétal inc. n'a pas, à date, l'intention d'explorer de façon intensive les terrains sans option et prétend ne pas se rendre au paiement final des options. 
En ce qui concerne la propriété Dalquier, les quatre titres miniers sous option nécessiteraient d'ici huit mois le versement de 50000 actions de la compagnie aux propriétaires à intervalles fixes. Aussi, l'entreprise a l'intention de vérifier leur potentiel avant juin 1988, afin d'éliminer cette option si aucune minéralisation n'est rencontrée.

Comparēe à d'autres entreprises d'exploration, Normétal inc. n'a pas d'obligations de travaux à rencontrer et a des obligations financières infimes à rencontrer envers les options signées à date. L'entreprise est réellement propriētaire de ses terrains miniers.

\subsubsection{3 règles de base dans le choix des propriétés}

Tous les terrains miniers sont localisés à l'intérieur de camps miniers établis. A l'exception du terrain Brouillan, tous les terrains sont dans les camps miniers de Noranda et de Val d'Or. Les propriétés sont toutes localisées à l'intérieur d'une ceinture volcano-sédimentaire. A l'exception de la propriété Brouillan, toutes les propriētés recèlent des minēralisations afin d'indiquer de façon certaine leur potentiel. Toutes sont en territoire accessible par un chemin carrossable pour réduire les coûts relatifs à leur exploration afin d'indiquer de façon certaine leur potentiel.

\subsubsection{2 propriēté La Sarre, Brouillan, Dal quier et Bassignac}

Ces propriétés doivent être considérées comme peu avancées. Elles vont nēcessiter des fonds importants pour y quantifier des minēralisations. Leur potentiel est élevé en raison de leur proximité des gisements en exploitation.

A 1 'exception de Brouillian, toutes ces propriétēs recèlent des minéral isations nombreuses qui mëritent un suivi. 


\subsubsection{3 propriété Barraute}

Les résultats du premier programme d'exploration furent intēressants. La propriété est sise entre plusieurs gisements et contient. de nombreuses minéralisations aurifères.

L'objectif du programme d'exploration de cette année est de prouver la continuité de ces minéralisations et de les quantifier, faire des réserves.

D'ailleurs, ce terrain a suscité l'intērêt d'autres entreprises. En effet, Normétal inc. a reçu des offres d'association, mais n'y a pas donnē suite.

\subsubsection{4 propriétẻ Normétal}

\subsubsection{1 gite Normetmar}

Une étude de préfaisabilité a permis d'étudier trois scénarios pour la mise en valeur du projet minier Normetmar. Le corps minëralisé de Normetmar est de forme allongée et plutôt lenticulaire. Il a un pendage d'environ $70^{\circ}$, une épaisseur d'environ 3.5 mètres et une largeur variant de 50 à 100 mètres. La lentille est observable en surface jusqu'à une profondeur de 425 mētres. Le calcul des réserves effectué par monsieur G.M. Hogg en avril 1970 indique la présence de 306800 tonnes à une teneur de $10.96 \%$ en zinc et $10 z /$ tonne d'argent.

La mise en valeur de ce dépôt fut étudiée sous trois approches différentes par la firme St-Michel Géoconseil. 


\subsubsection{2 historique du gisement Normetmar}

La mine Normétal fut en opëration de 1937 à 1975. Au début des années 60, Kerr Addison inc. a acquis la mine et l'a exploitēe jusqu'à sa fermeture. En 1967, l'opérateur exēcuta des travaux d'exploration pour découvrir des nouvelles réserves et éviter la fermeture de la mine.

Kerr Addisson inc. mit à jour un dépôt de zinc marginal (306 $800 \mathrm{t}$ à une teneur de $10.96 \%$ en Zinc) à 3000 pieds des infrastructures de 1 'ancienne mine.

En 1970, les géologues d'exploration recommandèrent d'aller sousterre pour augmenter le dépôt marginal et aussi récupérer le minerai. Le gérant de la mine, monsieur Ross Allen, refusa d'aller de 1 'avant dans le projet, sous prétexte que l'entreprise avait suffisamment explorë le-secteur et n'avait pu manquer un gisement de la dimension de la Mine Normétal (14 millions de tonnes). Il rejeta aussi l'idēe de miner le dépōt à cause de problèmes techniques et de la rentabilité douteuse du projet.

Au début des années 80, le régime des actions accréditives fait son apparition. Grâce à l'abri fiscal, il est maintenant plus facile de lever l'argent nēcessaire au projet d'exploration. Lé régime accréditif considère les travaux de mise en valeur (préparation de la mine) comme des dépenses d'exploration. Cela incite les compagnies d'exploration à s'intéresser à des projets marginaux qui auparavant, furent classés non économi ques.

En 1985, Ressources Audrey inc. s'intéressa au projet Mobrun. Il s'agit d'un dépôt de métaux de base marginal comme le projet Normetmar. L'entreprise réussit à financer son projet (13 
millions), à réaliser ses travaux et à dégager un profit de son opération. Récemment, elle annonçait une décision de production.

A partir de cet exemple, Normétal inc. a décidé d'étudier son projet Normetmar en fonction des possibilités du système accréditif, des prix élevés des métaux de base et des nouvelles techniques de minage.

\subsubsection{3 ëtudes sur le gisement}

A date, le service d'exploration de Kerr Addisson inc. a produit une ētude sur le projet. Il s'agit d'une ētude interne très limitée. Celle-ci proposait quatre scénarios avec des coûts ētablis à partir de "règle de pouce".

La faisabilitē technique était incomplète, de même que la faisabilité financière. D'ailleurs, le projet n'a jamais reçu 1 'approbation du gërant de la mine.

En 1987, Normētal inc. commanda une étude de préfaisabilité technique à la firme St-Michel Géoconseil inc. pour reconsidérer le projet avec la situation de 1987. Cette firme a suggērē les trois scēnarios suivants:

Scénario 1: réhabilitation du puits Normétal et échantill lonnage en vrac sur Normetmar

La réhabilitation du puits Normétal consiste à accompl ir le dénoyage jusqu'au niveau 1700 pieds et à traiter les eaux de pompage. De plus, il faut faire toutes les installations de surface, telles que le treuil, le chevalement, les compresseurs, etc. Sous terre, les travaux consistent à développer les niveaux 300,800 et 1400 de 1 'ancienne mine Normētal. 
Du point de vue de T'environnement, le traitement des eaux acides ( $\mathrm{PH} 4$ ) peut causer certains problèmes. L'échantillonnage en vrac est de 30000 tonnes et est miné après avoir enlevé les $60^{\prime}$ de mort-terrain sur la zone de Normetmar. Comme pour les autres scénarios, le minerai sera traité au concentrateur de Minéraux Noranda inc., division Matagami.

Scénario 2: fonçage d'un puits et échantillonnage en vrac

Ce scénario comprend le fonçage d'un puits jusqu'au niveau 1650 ' et le développement de plusieurs niveaux et sous-niveaux. Ici encore, 1 'échantillonnage en vrac est de 30000 tonnes et est miné par 1 a surface après avoir retiré le mort-terrain.

Scénario 3: échantillonnage en vrac de 68000 tonnes

Dans le cas du scénario 3, une partie de l'échantillonnage en vrac (38 000 tonnes) se fait en développant une rampe jusqu'au niveau $150^{\prime}$ et une galerie de forage au niveau 70'. L'autre 30000 tonnes d'échantillon est pris à la surface après avoir entevé le mortterrain.

\subsubsection{4 méthode de minage du scénario 3}

Comme le scénario 3 semble le plus réalisable financièrement, nous dēcidons de ne pas présenter les méthodes de minage des scénarios 1 et 2 , ainsi que leur évaluation financière.

Echantillonnage en vrac de 68000 tonnes

La méthode pour échantillonner 38000 tonnes de minerai consiste à foncer une rampe jusqu'au niveau $150^{\prime}$ à une pente de $15 \%$ (figure IV). Au niveau $70^{\prime}$, une galerie de forage est développée et des 
trous de 2" de diamètre d'une cinquantaine de pieds de longueur sont forés vers le bas et vers le haut. Au niveau 150', des trous d'une trentaine de pieds sont forés vers le haut. Des chargeusesnavette $5 \mathrm{vg}^{3}$ soutirent le minerai et un camion de $13 \mathrm{t}$ (ou $26 \mathrm{t}$ ) est utilisé pour transporter le minerai à la surface. L'autre échantillon de 30000 tonnes est exploité par la surface après avoir enlevé le mort-terrain.

\subsubsection{5 évaluation financière du scénario 3}

Scénario 3: échantill onnage en vrac

Ce scénario comprend cinq phases, soit:

Phase 1: $\quad$ Forage de définition et échantillonnage en vrac de surface

Phase 2: $\quad$ Préparation du site

Phase 3: Installation de surface

Phase 4: Installation des infrastructures souterraines

Phase 5: Echantillonnage en vrac sous-terre

Phase 1: Forage de définition

1. Forage de définition du pilier de surface 5000 pieds à $25 \$ /$ pied (tout inclus)

$125000 \$$

2. Forage de définition 15000 pieds à $25 \$ /$ pied (tout inclus)

$375000 \$$

3. Décapage 60000 tonnes à $3 \$$ tonne $180000 \$$

4. Echantilion en vrac de surface 30000 tonnes à 7 \$/tonne

5. Transport à Matagami 30000 t.c. à 8 \$/tonne $240000 \$$

6. Concentrateur 30000 t.c. à $13 \$ /$ tonne $390000 \$$ 
7. Raffinage 3976 t.c.h. de concentré de zinc à $280 \$ /$ tonne

8. Transport à Valleyfield 3976 t.c.h. à $40 \$ /$ t.c.h. $159000 \$$

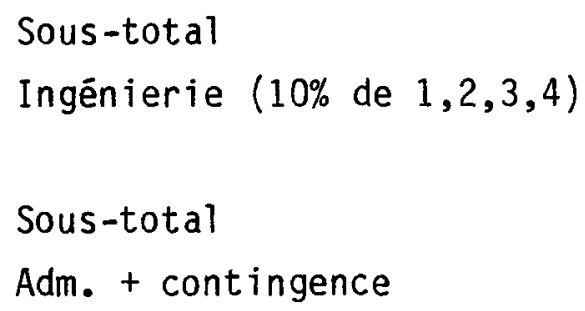

Total (phase 1)

$3313000 \$$

Phase 2: Préparation du site

Demande de permis et étude environnementale $75000 \$$ Route d'accès et coupe de bois $5000 \$$ Bassin de sédimentation $15000 \$$ Source d'eau potable; pompe $25000 \$$ Hydro-Québec; raccordement $60000 \$$

Construction de surface

- office, sécherie $45000 \$$

- électricité de surface $20000 \$$ Arpentage $20000 \$$ Télécommunication $10000 \$$

$$
\begin{aligned}
& \text { Sous-total } \\
& \text { Ingēnierie (10\%) } \\
& \text { Sous-total } \\
& \text { Adm. + imprēvus (15\%) }
\end{aligned}
$$$$
275000 \$
$$$$
27500 \$
$$

$302500 \$$

$45 \quad 500 \$$

Total (phase 2) 


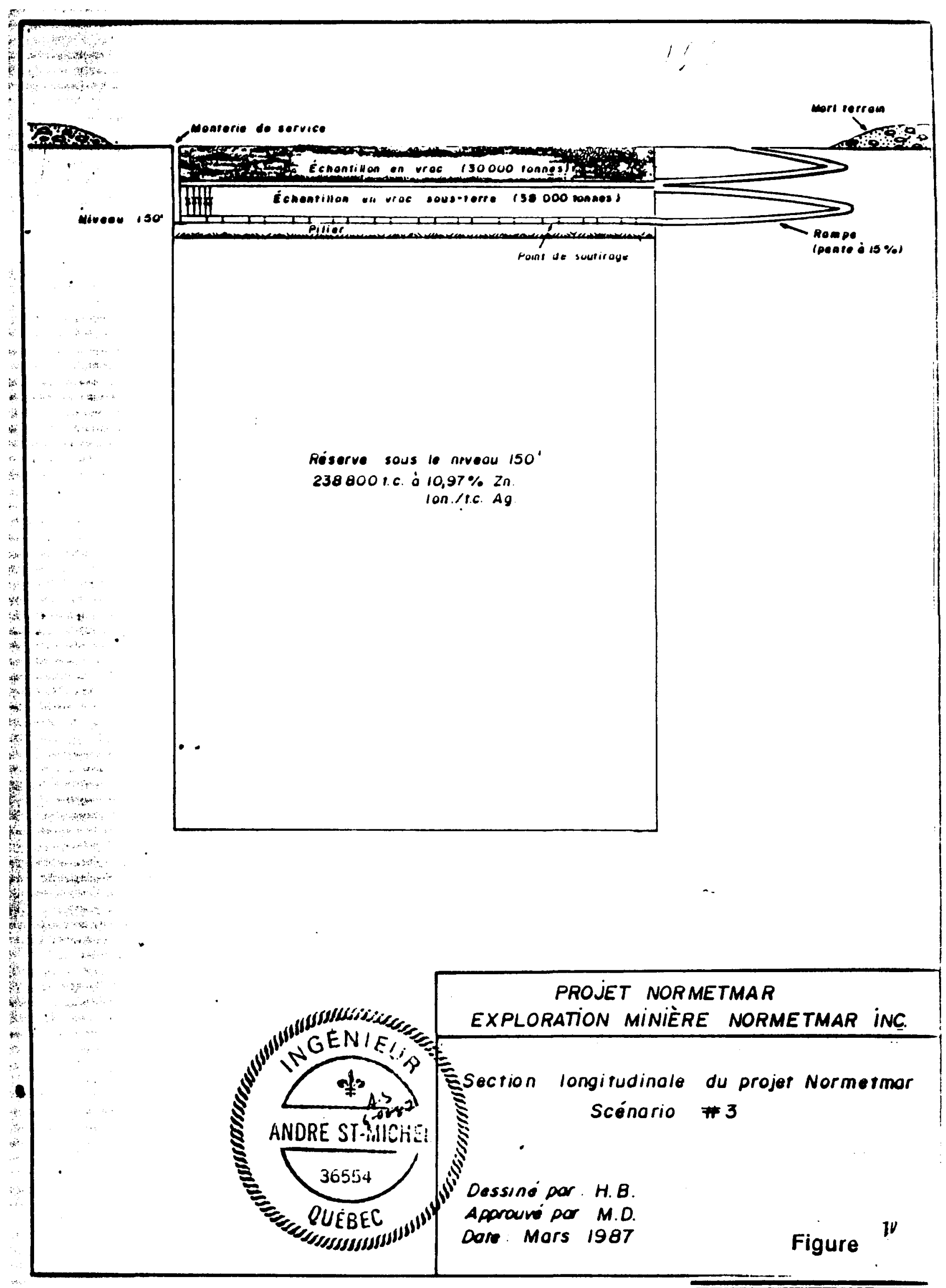

siil Mictholl 
Phase 3: Installation de surface

Garage

$25000 \$$

Sous-total

$25000 \$$

Adm. + contingence (15\%)

$4000 \$$

Total (phase 3)

$29000 \$$

Phase 4: Installation des infrastructures souterraines

Rampe à $15 \%$ de pente: 1000 pieds à 600 \$/pied $\left(14^{\prime} \times 12^{\prime}\right)$

$600000 \$$

Galerie de forage $12^{\prime} \times 9^{\prime}: 600^{\prime}$ à $400 \$ / p i e d$

$240000 \$$

Galerie de halage $9^{\prime} \times 14^{\prime}: 350^{\prime}$ à $500 \$ / p i e d$

$175000 \$$

Point de soutirage $9^{\prime} \times 9^{\prime}$ (à tous les 30')

300 pieds $\times 380 \$ /$ pied

$114000 \$$

Cheminée d'ouverture $6^{\prime} \times 6^{\prime} ; 150^{\prime} \times 300 \$ /$ pied

$45000 \$$

Ventilation: 40000 p.c.m. 2 moteurs de 75 HP

$75000 \$$

Chauffage

$50000 \$$

Sout-total

$1299000 \$$

Ingénierie $(10 \%)$

$130000 \$$

Sous-total

$1429000 \$$

Adm. + imprēvus (15\%)

$214000 \$$

Total (phase 4)

$1643000 \$$ 
Phase 5: Echantillonnage en vrac sous-terre

Dépenses générales de mine

38000 tonnes $\times 2 \$ /$ tonne

$76000 \$$

Echantillonnage de 38000 tonne

méthode sous-niveau retraité 7 \$/tonne

$266000 \$$

soutirage et transport jusqu'à la surface

$10 \$ /$ tonne

$380000 \$$

Transport à Matagami

38000 tonnes à $8 \$ /$ tonne

$304000 \$$

Concassage primaire à Matagami

38000 tonnes à $2 \$$ tonne

$76000 \$$

Concentrateur: 38000 tonne à 13 \$/tonne

$494000 \$$

Raffinage: 5036 t.c.h. de concentré de zinc

à $280 \$ /$ t.c.h.

$1410000 \$$

Transport à Valleyfield

5036 t.c.h. à $40 \$ /$ t.c.h.

$201000 \$$

Sous-total

Ingēnierie ( $10 \%$ de 1 et 2$)$

$3207000 \$$

$34000 \$$

Sous -total

$3241000 \$$

Adm. + imprévus (15\%)

$3486000 \$$

Total (phase 5)

$3727000 \$$

GRAND TOTAL (phases $1,2,3,4$ et 5)

$9060000 \$$

\subsubsection{6 recommandations de 7 'expert-conseil}

Cette étude de préfaisabilité a permis aux promoteurs du projet de voir trois scénarios de mise en valeur du gîte Normetmar. A la 
suite des études techniques et financières contenues dans ce rapport, il ressort que:

- la réhabilitation du puits de Normétal ne peut être justifiée économi quement:

$$
\text { Coût total du projet }
$$$$
\text { Revenus bruts espérés }
$$

Perte
$43314000 \$$ $24596000 \$$

$18718000 \$$

- de plus, les problèmes susceptibles de survenir lors du traitement des eaux acides rendent ce scénario peu intéressant;

- le fonçage d'un puits est également une option peu intéressante puisque très difficile à justifier sur le plan économique. En effet:

$$
\text { Coût total du projet }
$$$$
\text { Revenus bruts espérēs }
$$

Perte
$42365000 \$$

$24596000 \$$

- l'échantillonnage en vrac de surface et sous-terre demeure le scénario le plus intéressant, car il est faisable tant sur le plan technique que financier

$\begin{array}{lr}\text { Coût total du projet } & 9060000 \$ \\ \text { Revenus bruts espérés } & 5452000 \$ \\ \text { Perte } & 3608000 \$\end{array}$

Les recommandations sont donc les suivantes: 
- il faut intensifier le programme d'exploration de façon à rehausser la quantité et la qualité des réserves;

- il faut mettre en valeur le gîte minier Normetmar à l'aide du scénario 3 (échantillonnage en vrac de surface et sous terre). Le programme est distribué sur cinq phases et s'échelonne sur une période d'un an.

Le financement de ce rapport devrait pouvoir être réal isé à $100 \%$ par des actions accréditives. Ainsi, si l'on néglige la dilution du capital-actions de 1 a compagnie, $i 1$ y a un profit possible final net et non actualisé de $5452000 \$$ à la fin de la première année.

\subsubsection{5 résidu minier de Normétal}

\subsubsection{1 étude quantitative sur le résidu minier}

En 1985, Exploration minière Normétal inc. a commandè à un expertconseil indépendant une étude pour déterminer le tonnage du parc à rēsidu minier contenu sur la propriété Normétal. Monsieur Serge Bari1, B. Sc. A. en génie minier, professeur en traitement des minerais au Département de technologie minérale du Collège de l'Abitibi-Témiscamingue, a produit l'étude.

\subsubsection{2 résultats de 7 'étude}

Tonnage du parc à résidu: 8848997 tonnes courtes

Quantités restantes: $\mathrm{Cu}=15122$ tonnes courtes

$Z n=76249$ tonnes courtes

$A u=87961$ oz troy

$\mathrm{Ag}=5255440$ oz troy

Autres (gangues)

$=8595760$ tonnes courtes 


\subsubsection{3 étude de faisabilité}

En 1987, Normétal inc. a signé une entente avec Doelcam inc. pour l'évaluation du parc à résidu minier. Doelcam inc. est une entreprise privée ontarienne qui possède l'expertise dans ce genre de projet.

\subsection{I'entente avec Doel cam inc.}

Le partenaire doit produire une étude de faisabilité avant le 31 janvier 1988. Le coût de l'étude variera entre $150000 \$$ et $500000 \$$ en raison du nombre de tests métallurgiques et du nombre de forages dans notre résidu (pour reconfirmer le tonnage et les teneurs).

A la suite des résultats, Doelcam inc. fera une proposition de coparticipation pour mettre sur pied une opération de récupération.

L'entreprise de coparticipation prévoit que Normétal inc. et sa filiale absorberont $50 \%$ des coûts et recevront $50 \%$ des profits. Doelcam inc. sera l'opérateur du projet et sera rétribuée pour son expertise en tant qu'opérateur de l'entreprise de coparticipation.

\subsubsection{5 décision de production}

"Ce n'est pas tous les jours qu'une "junior" peut s'offrir une étude de faisabilité sur un projet d'exploitation minière". Cet événement arrive lorsque les phases de l'exploration et de l'évaluation sont terminées. Il faut alors réaliser la phase du développement (accès au gisement). Le temps nécessaire pour realiser ces trois phases est d'un minimum de 5 ans et les investissements sont de 1 'ordre de 15 à 25 millions de dollars. Les étapes qui suivent sont la construction et 1 a production. Les compagnies franchissant les 
trois premières étapes sont peu nombreuses et ont, en général, entre 10 et 20 millions d'actions sur le marché. Celles qui franchissent les cinq étapes sont encore moins nombreuses...

Dans un projet de "tailing", la phase de développement n'existe pas ( 1 'amoncellement est à la surface). Les phases de l'exploration (forage) et de l'évaluation (essais métallurgiques) sont exécutées avec une fiabilité impressionnante qui permet une décision de production en toute sécurité. Doelcam inc. se chargera des phases de construction et de production en tant qu'opérateur. Comme nous l'avons dit, elle a l'expertise nécessaire et procédera selon une cédule serrée (18 mois pour toutes les phases).

Au moment de la rédaction de ce rapport, Doelcam inc. avait procédé au forage du résidu. Les tests métallurgiques sont débutés et les résultats prêliminaires sont positifs.

\subsubsection{6 liquidités et placements}

Au moment de 1 a rédaction de ce rapport, Exploration minière Normétal inc. a des liquiditēs de 200000 \$qu'elle entend conserver pour saisir une opportunité.

L'entreprise possède un placement important dans Exploration minière La Sarre inc., soit 750000 actions. Les actions de la société La Sarre seront cotées en Bourse de Montréal à 0,50\$. A ce moment, la valeur du placement sera de $375000 \$$. Son potentiel de croissance sera important et sera fonction des résultats des programmes d'exploration de La Sarre inc. au cours des années.

Normétal inc. peut disposer de son placement dans La Sarre inc. de la façon suivante: 


\begin{tabular}{|c|c|}
\hline $20 \% \quad\left(\begin{array}{lll}75 & 000 & \$\end{array}\right)$ & en septembre 87 \\
\hline $10 \%(37500 \$)$ & en septembre 88 \\
\hline $10 \%(37500 \$)$ & en septembre 89 \\
\hline $10 \%(36500 \$)$ & en septemt \\
\hline
\end{tabular}

(187500 \$) s'il y a une découverte effectuée par La Sarre inc.; $20 \%$ lors de 1 a mise en production.

Aussi, en dedans de 12 mois, Normétal inc. peut compter sur $112500 \$$ d'argent liquide supplémentaire pour saisir une opportunité par la vente d'un certain nombre d'actions de La Sarre inc.

\subsubsection{7 qual ité des actifs}

\subsubsection{1 effet des cycles sur les actifs miniers}

Depuis la fin des années 70 , nous sommes dans un cycle haussier pour l'or. Auparavant, les métaux de base ont connu un cycle favorable qui a duré 20 années approximativement.

Il est important que le portefeuille de propriétés soit en concordance avec les effets cycliques des métaux. La confection d'un bon portefeuille de propriētés nécessite du temps et de 1 'argent.

I1 existe approximativement 10000 prospects miniers au Canada. Donc, la sēlection des meilleurs et leur acquisition demandent du "savoir-faire" et lient la compagnie pour plusieurs années. Si le terrain ne lie pas la compagnie junior, il lui coûte des fonds importants en travaux d'exploration.

Le portefeuille de propriétés minières de Normétal inc. est suffisamment diversifié (or et métaux de base) pour ne pas être déprēcié par l'effet des grands cycles dans les prix des métaux. 
Ce portefeuille de propriétés n'est pas grevé d'hypothèques (versements et obligations de travaux). Il ne constitue donc pas une source de drainage des fonds actuels et à venir de l'entreprise.

Deux propriétés sont à des stades plus avancés: Normétal et Barraute. Sur la propriēté Normétal, l'entreprise peut compter sur des minéralisations quantifiées (gîte de zinc et résidu minier), ce qui est assez rare.

\subsubsection{2 santé financière}

La moitié du placement dans La Sarre inc. peut être rendu liquide au cours des prochaines annēes sans difficulté. L'autre moitié n'est pas très liquide, mais va se refléter dans les états financiers.

A très court terme, l'entreprise peut compter sur un total de $312500 \$$ de liquiditēs. A court terme, l'entente avec la filiale La Sarre inc. permet à Normétal inc. d'obtenir tous les fonds nécessaires pour l'exploration des propriétés. La majorité des charges administratives sont incluses dans les budgets d'exploration en fonction de l'entente d'exploration.

\subsection{LE CLIMAT ORGANISATIONMEL}

\subsubsection{Mission de 1 'entreprise}

Compte tenu de la structure simple de l'entreprise, toutes les activitēs sont effectivement effectuées par rapport à la philosophie de base de l'entreprise. Jusqu'à maintenant, l'entreprise a été fidèle à sa mission d'exploration. Les structures établies servent cette mission. A titre d'exemple, le personnel recruté a 
effectivement les habiletés nécessaires pour réaliser les activités d'exploration et non de production (minage).

\subsubsection{Influence du système client}

Pour le moment, on peut dire que les relations entre les différents intervenants sont bonnes. Si les actionnaires représentent le système client et le service d'exploration, la production, il n'y a pas eu, jusqu'à maintenant, de zone conflictuelle entre les deux systèmes pour 1 'obtention de résultats positifs. Plus l'entreprise vieillira, plus les pressions seront fortes pour obtenir des résultats significatifs de 1 'exploration et, par le fait même, de la performance du titre.

\subsubsection{Relations de travail}

Au niveau des relations de travail, le climat est acceptable. La culture propre au milieu de l'exploration est présente. Cela signifie que les techniciens et professionnels considèrent comme normal la prolongation de la semaine de travail et des heures quotidiennes en fonction des activités.

Jusqu'à maintenant, l'entreprise a un style de gestion par fonction. Elle a choisi de former ou de constituer sa propre équipe de techniciens et géologues pour être moins dépendante des consultants.

Récemment, l'entreprise a implanté une gestion par projet au niveau du service de l'exploration. Ceci a eu pour effet d'éviter que toutes les activités d'exploration soient dépendantes d'une seule personne. Ceci s'avère indispensable, car l'entreprise aura plus de projets et plus de personnel en exploration cette année. 


\subsubsection{Perspectives}

L'existence même du système accréditif étant menacé, cela influe sur la sécurité d'emploi de toutes les ressources de l'entreprise. Hors du système accréditif, l'entreprise pourrait recueillir beaucoup moins d'argent auprès du public pour ses projets d'exploration. La conséquence apparaît évidente sur le climat organisationnel. Le niveau d'activité, le nombre de ressources humaines ainsi que les rémunérations seraient rēduits. La majorité des dirigeants et des empioyés devraient travailler à temps partiel. L'entreprise devrait utiliser les contractuels plus fréquemment.

\subsection{LA ZONE PROBLEMATIQUE}

\subsubsection{Interrel ation des problèmes}

Une situation problématique complexe se caractérise souvent par 1 'existence de plusieurs perspectives, par une absence de relation causale simple, par la répercussion d'un problème sur un autre, par la présence de plusieurs acteurs et intervenants, par la présence de plusieurs critères de performance et par une difficultē de quantifier.

Nous sommes ici en présence d'une situation problématique complexe. Se centrer uniquement sur une perspective risque d'amenuiser notre zone problématique; c'est pourquoi il est important d'adopter une perspective plus globale et de retenir plusieurs types de problèmes.

Il est évident qu'un premier type de problème concerne 1'acquisition des terrains miniers. Par la suite, survient le problème de trouver 
les fonds pour explorer les terrains et, enfin, la difficulté de valoriser les terrains par des découvertes.

Ainsi, les problèmes présentés de façon séquentielle se présentent de la façon suivante: acquisition des terrains miniers, financement des travaux d'exploration et exécution des travaux d'exploration par les ressources humaines de l'entreprise.

\subsubsection{Problèmes d'acquisition}

Tous les terrains ne sont pas de même qual ité et de même prix. De plus, l'entreprise a des limites physiques et temporelles en fonction de l'éloignement des terrains et en fonction des ressources humaines et financières pour réaliser ses travaux d'exploration. Aussi, certains terrains connaissent des niveaux d'avancement différents en fonction des travaux antérieurs.

L'objectif ultime est de mener un projet (terrain) au niveau de la production. Le choix des terrains s'effectue en fonction de certaines réalités: disponibilité des terrains, disponibilité des fonds, accessibilitē, connaissance gēologique, travaux antērieurs, potentiel géologique, engouement (rush).

\subsubsection{Problèmes de financement}

L'entreprise possède deux types de clients. Sur le marché primaire, l'investisseur recherche d'abord l'abri fiscal. Sur le marché secondaire, le spéculateur recherche le gain rapide.

Une performance boursière sur le marché secondaire facilite la vente de nouvelles actions lors d'une émission primaire. A ce moment, 1 'entreprise obtient ses fonds plus facilement. Donc, la production de l'entreprise ne peut qu'attirer l'attention des investisseurs. 
Plus elle sera remarquëe, plus elle aura de la facilité à recueillir des fonds.

Le financement dépend aussi de certaines réalitēs: résultats (découvertes), prix de l'action, force de vente des courtiers, raretē des titres, abondance des acheteurs.

Il est évident que la performance boursière dépend en partie de la promotion, ou plutôt du marketing de l'entreprise. Ainsi, selon le niveau d'avancement des propriētēs, le produit sera différent et influencera le "mix marketing" de 1 'entreprise.

\subsubsection{Problèmes de performance des ressources humaines}

Les problèmes de sélection des propriētés et de financement sont intimement 1 iés à 1 'expertise dans le domaine des sciences de la terre, du marketing et du financement des dirigeants.

La qualité des décideurs rêside dans leur habileté à dénicher les bonnes propriétés, leur habileté à financer facilement les travaux et leur habileté à faire progresser les terrains vers des niveaux de projets plus avancēs.

La capacité d'attirer des spēcialistes des sciences de la terre au service de 7 'entreprise est importante. En effet, 7 'exploration est devenue sophistiquée et utilise des technologies de pointe. L'entreprise doit se démarquer de l'exploitation artisanale pour augmenter sa performance technique, soit la dēcouverte de gites minéralisés. 


\subsubsection{Choix du problème et justification}

La ressource minërale est toujours cachëe dans les entrailles de 1a terre. Le processus de transformation définit la nature même de 1 'entreprise. Retenir uniquement un type de problème risque de biaiser, d'orienter l'entreprise vers des solutions partielles. Les problèmes retenus sont donc la politique d'acquisition des propriétés minières, la mise en place d'une politique de marketing et la mise en place d'une politique de planification et d'utilisation des ressources humaines.

Des lieux d'amélioration potentielle sont les critères retenus pour la sêlection des problèmes. Ces trois problèmes nous apparaissent comme des opportunités de développement parmi tous les points litigieux. 


\section{CONCLUSION}

Une meilleure connaissance de notre entreprise est maintenant acquise. L'environnement comprend les variables incontrôlables qui influent sur l'entreprise. Dans le système ètudié, les structures formelles de 1 'entreprise ont été expliquées. L'étude du processus de transformation est une synthèse des opérations réelles de l'entreprise. Avec l'étude des soussystèmes, les ressources de l'entreprise ont été décrites. Ces ressources sont les possibilitēs financières et technologiques, les fonctions marketing et personnel et les actifs. Le climat organisationnel a ét examiné à travers le système client et ses répercussions sur la mission de l'entreprise.

Le constat général met en évidence la complexité de l'environnement de l'entreprise. Ce même environnement influe de façon importante sur la performance de 1 'entreprise.

Les sous-systèmes, et particulièrement le marketing, ont aussi une influence très importante sur notre organisation. La notion de produit est particulièrement surprenante et met en perspective la nécessité ou 1 'importance du marketing. Nous avons ainsi identifié un noeud de problèmes. 
TROISIEME PARTIE

ELABORATION D'UNE SOLUTION 


\section{INTRODUCTION}

Cette troisième partie du mémoire de recherche-action porte sur le choix d'un système d'activités capable d'améliorer la situation problématique décrite dans 1 a deuxième partie.

Un système de planification stratégique permettant à l'entreprise d'effectuer des changements dans un processus de résolution de problèmes est ici proposē. Il s'agit d'un modèle conceptuel. Ce modèle est utilisé après avoir examiné ce que certains auteurs pensent de la planification stratégique. Il est utilisé en fonction de l'analyse et de la connaissance que l'acteur a de I'entreprise. 


\subsection{LA PERSPECTIVE}

La perspective est déterminante lors de l'étude ou de l'analyse d'une situation problématique sēlectionnēe. Le choix de la perspective oriente l'élaboration de la solution en fixant les paramètres de travail.

\subsubsection{Systèmes et sous-systèmes}

Notre entreprise constitue notre système. Comme toute entreprise, un certain nombre de sous-systèmes lui permettent de fonctionner. Généralement, les fonctions traditionnelles de l'entreprise correspondent aux sous-systèmes: personnel, finance, production, marketing.

Dans le cas de notre entreprise, nous avons déterminé un certain nombre de sous-systèmes en relation avec le processus de transformation. Les sous-systèmes "ressources humaines" et "finances" correspondent aux fonctions personnel et finance. Les sous-systèmes "technologie" et "actifs" correspondent ou se rapprochent de la fonction de production. Le sous-système "marketing" correspond évidemment à la fonction marketing.

Les sous-systèmes constituent des parties et sont toujours interreliés. Pris individuellement, ils sont essentiels à 1 'entreprise et influent sur le fonctionnement des autres soussystèmes. Donc, une zone d'amélioration dans 1 'un ou plusieurs des sous-systèmes va provoquer des changements en chaîne. C'est dans cette perspective ou avec ce point de vue que nous envisageons des solutions à la problématique. 
3.2 CHOIX D'UN SYSTEME D'ACTIVITES PERTINENT AU PROBLEME

Le choix du système pour les problèmes sêlectionnēs est le suivant: un système d'activités pour la planification stratégique assurant une performance optimale en fonction des projets de l'entreprise.

\subsubsection{Examen de la littérature}

Alain Charles Martinet, dans son volume Management stratégique: organisation et politique, effectue une revue de littérature et analyse les principaux concepts propres à la stratégie. Il met en perspective les deux approches stratégiques retrouvées dans la littérature. La figure $V$ illustre ces deux tendances. 


\section{FIGURE V: APPROCHES STRATEGIQUES}

1. L'entreprise est un organisme technicoéconamique.

2. L'environnement de T'entreprise est essentiellement le(s) marché(s) sur le(s)quel(s) s'affrontent des concurrents.

- "L'entreprise est en marchē(s)".

3. La stratégie est celle de l'entreprise en lutte (et accessoirement en coopération) avec les acteurs intervenant sur le(s) marchē(s).
1. L'entreprise est simultanément:

- un agent de production,

- une organisation sociale,

- un système politique.

2. L'environnement est constitué de tous les acteurs en relation (effective et potentielle) avec l'entreprise -- et de 1 'ensemble "résiduel" (groupes d'opinions, relais...) qui influence les attitudes et comportements des premiers. - "L'entreprise est en sociétê".

3. La stratëgie est d'abord celle que le "groupe dirigeant" choisit pour $l^{\prime}$ institution dont il a la garde, en lutte-coopération avec les acteurs internes et externes concernés. Elle devient celle de l'entreprise lorsqu'elle est prise en charge par son corps social.

4. La stratégie consiste à créer les conditions de congruence -économiques, techniques, sociales, politiques -- entre l'environnement et 1 'entreprise de sorte que celle-ci dispose d'un potentiel maximum de performances.

5. L'effort de réflexion stratégique porte sur le choix d'un positionnement judicieux de l'entreprise dans 1 'environnement ET sur les conditions de son acceptation par les acteurs internes, externes et "résiduels". - L'analyse stratégique, récusant la séparation formulation/mise en oeuvre, porte "ex ante" sur la compatibilité du projet avec les éléments freins et moteurs.

6. Ces èléments sont envisagés selon la double logique des facteurs et des acteurs (p. ex.: quantité et qualité de travail et comportements/pouvoirs des "travail leurs".

- La rationalité stratégique est de type mixte (technico-ēconomique socio-politique).

Source: A.C. Martinet, Managenent stratégique: organisation et politique, Mc Graw-Hill, 1984, p.33. 
Selon l'approche classique, l'entreprise est une machine organisationnelle qui recherche la maximisation du profit dans un univers concurrentiel grâce à des processus de transformation et d'èchange.

Martinet rapporte que $H$. de Bodinat et $V$. Mercier dans "L'analyse stratégique moderne" et J. Lecaillon dans "Analyse microéconomique" traitent de la stratégie dans une perspective microéconomique. Les entrēes sont transformés en sorties. L'allocation des ressources est fonction du positionnement recherché sur le marché.

Selon 1'approche rénovée, l'entreprise demeure un fournisseur de biens et services et reçoit à cet effet une sanction de la sociēté. La sanction est donnée par le marchē et aussi par l'Etat qui légitimise l'activité et l'existence utilitaires de l'entreprise. L'entreprise doit compter avec des acteurs externes autres que ceux normalement présents dans le marché.

L'entreprise est une société avec un jeu interne du pouvoir. Les dirigeants sont les responsables de la réalisation des objectifs de l'entreprise. Leur mandat est de permettre l'atteinte de ses buts et d'une performance. Il ne s'agit pas d'objectifs individuels transformés en objectifs organisationnels. Il s'agit d'une entité institutionnelle, un ensemble social, qui réalise ses objectifs.

L'organisation doit tenir compte de son environnement. L'entreprise exerce un pouvoir politique externe. Elle tente d'influer sur les règles, les acteurs et pratiques de l'environnement. Elle ne fait pas juste s'adapter, elle tente de modifier l'environnement en sa faveur. L'environnement est beaucoup plus large que le marché. Les évolutions technologiques culturelles et sociales influent sur le marchē concurrentiel et l'entreprise désire les influer. 
Parler de stratégie, sans parler de choix stratēgique, de planification stratégique, de mise en oeuvre stratégique et de gestion stratégique est inadmissible. Cependant, il est facile d'en discuter en rëférant aux deux conceptions de la figure $V$ et à des définitions générales.

Alain Charles Martinet présente ainsi la conception classique:

...la façon dont l'entreprise alloue ses ressources dans le but de modifier l'équilibre concurrentiel a son avantage. On sait qu'elle consiste essentiellement à définir les domaines d'activités dans lesquels l'entreprise s'engage, se maintient ou se développe, ceux dont elle se retire, l'intensité et l'ordonnancement de ces mouvements. 1

Les conceptions de Pierre Tabatoni et Pierre Jarniou de la stratégie se rapprochent de la conception de A.C. Martinet et s'apparente à 1 'approche rénovée:

Pour nous, de façon assez proche de Pierre Tabatoni et Pierre Jarniou, la stratégie désigne l'ensemble des critères de dếcision, choisi par le noyau, pour orienter de façon déterminante et sur la durée, les activités et la configuration de l'entreprise.?

Martinet continue sa définition générale de la stratégie en ces termes:

La stratégie est "externe" puisqu'elle cherche à mettre l'entreprise en situation d'insērer de façon profitable dans son environnement. ... une rationalité organisationnelle qui va guider, dans le temps, la cohérence des multiples décisions et

1. Management stratégique: organisation et politique, Mc Graw-Hill, 1984, p.48.

2. Citēs par Alain Charles Martinet dans Management stratégique: organisation et politique, Mc Graw-Hill, 1984,p.48. 
actions courantes. La stratégie, produit du noyau stratégique, trace donc les voies de la conduite simultanée de l'institution et du corps social, c'est-à-dire du gouvernement de l'entreprise, en s'appuyant sur les diverses dimensions de la réalité organisationnelle. 1

Ackoll, R.L. définit la planification stratégique comme suit: "La planification consiste à concevoir un futur désiré ainsi que les moyens réels d'y parvenir" 2.

Raymond Alain Thietart parle de la stratégie en ces termes:

La planification d'entreprise est un processus qui s'articule autour de plusieurs phases. Le processus débute par la fixation des objectifs, suivi de la formulation de la stratégie. Ensuite, viennent d'une part, l'établissement des politiques fonctionnelles de marketing, de production, de recherche, de personnel et, d'autre part, la construction de plans détaillés décrivant la manière d'atteindre ces objectifs. Enfin, un nouveau cycle de fixation d'objectifs, d'élaboration de la stratégie, de préparation des politiques et des plans d'action qui en découlent est mis en oeuvre en fonction des résultats atteints. 3

A travers ces définitions générales, nous retrouvons les notions de choix et de planification, deux concepts de la stratégie. Les deux conceptions (classique et rénovēe) font appel à un processus décisionnel. Cela signifie des choix pour les ressources, les parts de marché, etc. Les entreprises décident de leur avenir en établissant des plans d'action. Elles planifient. Elles se fixent

1. Management stratégique: organisation et politique, Mc Graw-Hill, 1984, p.48.

2. Cité par Michel Godet dans Prospective et planification stratégique, Editions Economica, 1985, p.168.

3. La stratégie d'entreprise. McGraw-Hi11, 1984, p.12. 
des objectifs, dēfinissent des activitēs et évaluent des résultats. Ainsi, les notions de planification stratégique et de choix stratégiques se retrouvent dans des prises de décision en relation avec la situation de l'entreprise.

La mise en oeuvre stratégique et la gestion stratégique se retrouvent uniquement dans ola conception rénovée de la stratégie. Ces notions sont apparues suite à l'émergence de l'entreprise comme corps social. L'entreprise est un regroupement d'individus et donne naissance à la spécialisation des activités et par le fait même à la hiéarchisation. En pratique, cela signifie que quelqu'un dans $l$ 'entreprise conçoit la stratégie et qu'un autre individu l'exécute soit le gestionnaire.

\subsubsection{Particularité de l'exploration minière}

La description de l'environnement, en deuxième partie, montre bien l'influence de 1 'environnement sur les entreprises d'exploratiron et plus particulièrement l'importance du système de financement grâce à la fiscalité. Cette situation s'applique à Exploration minière Normétal inc.

En première partie du mémoire, nous démontrons de l'importance de 1 'entrepreneurship dans les nouvelles entreprises d'exploration. Exploration miniēre Normétal inc. est une P.M.E. de petite taille. Cette P.M.E., comme beaucoup d'autres P.M.E., doit son existence à la volonté des propriétaires-dirigeants.

Donc, un modèle conceptuel se rattachant plus à l'approche stratégique classique est plus valable car il permet à Exploration minière Normétal inc. de mieux lire son environnement et de s'y adapter. A cause de la dimension de l'entreprise et de sa perrénité incertaine, il est plus pratique de concevoir un modèle stratégique 
inspiré de la conception classique de la stratégie. Un modèle inspiré de la conception rénovée se prête plus à une entreprise de grande dimension. Donc, notre modèle stratégique est un modèle adaptatif.

\subsubsection{Définition d'un modèle}

La planification stratégique est la résultante d'une analyse qui permet à la direction de Normétal inc. d'établir la meilleure harmonie possible entre les ressources de l'entreprise, les opportunités et les menaces de l'environnement et les aspirations des dirigeants pour solutionner les problèmes identifiés: la sēlection des propriétés, la promotion des projets de l'entreprise et la performance technique de ses ressources, et pour assurer la survie de 1 'entreprise.

\subsection{MODELE CONCEPTUEL}

Le choix du modèle permet une analyse rapide des opportunités et des menaces de l'environnement en fonction des forces et des faiblesses de l'entreprise et en relation avec les valeurs et les aspirations des dirigeants.

Le modèle permet de fixer un équilibre plus harmonieux entre 1 'environnement, l'entreprise et les décideurs. Il permet une analyse de la situation actuelle (où en sommes-nous maintenant?), de l'environnement (où pourrions-nous aller?), de nos aspirations (où voulons-nous aller?) et des possibilités offertes en fonction des ressources (où pouvons-nous aller?). 
FIGRE VI MOOEE CONCEPTIE DE LA PLANIFICATION STRATEGIQE

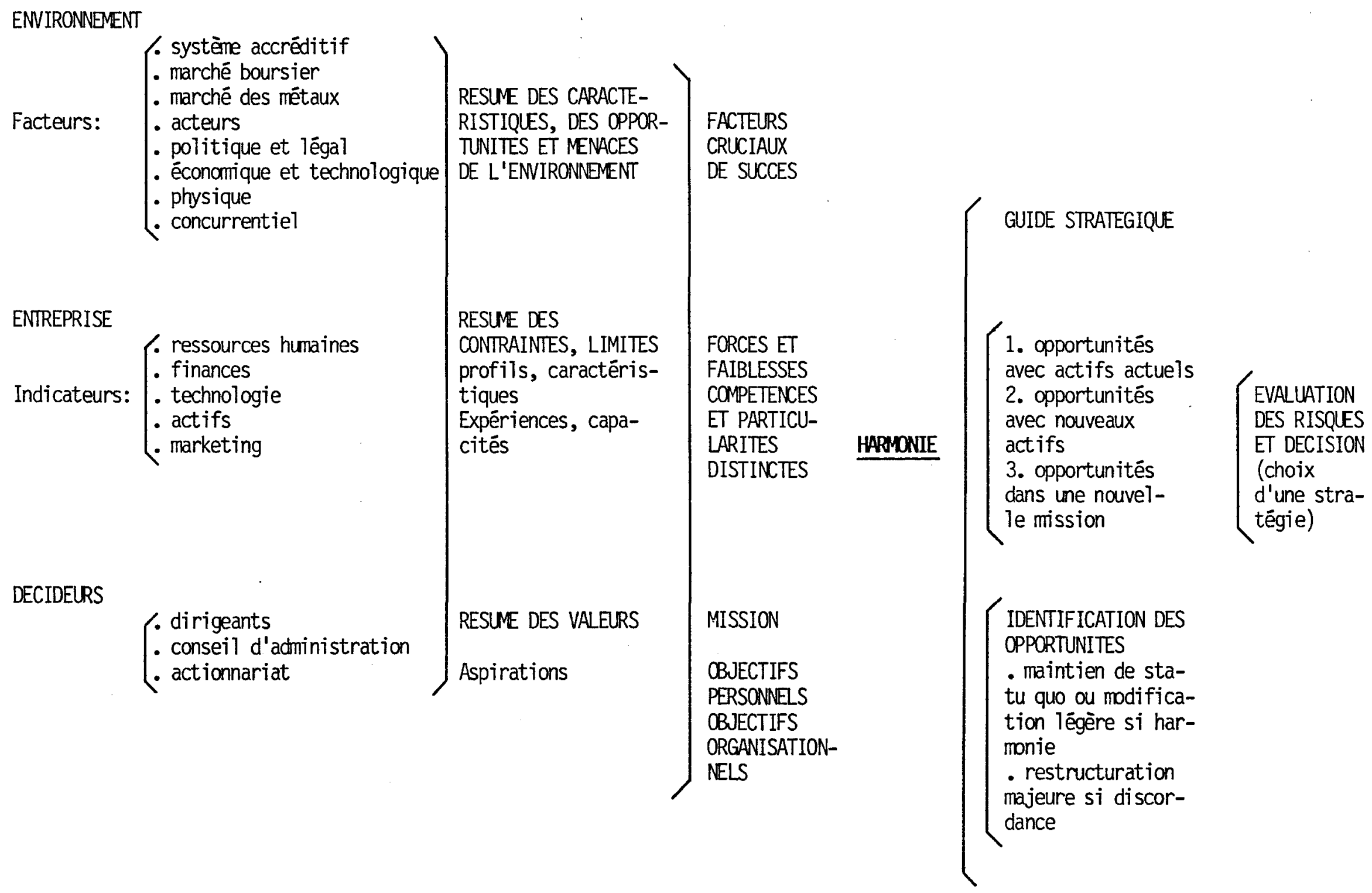




\subsubsection{Explication du modèle}

$\begin{aligned} \text { ENVIRONNEMENT } \quad=\quad \begin{array}{l}\text { un ensemble de facteurs qui a un impact sur } \\ l \text { 'entreprise. }\end{array} & \end{aligned}$

Facteur-système accrēditif $=$ programme qui permet des investissements dont le coût net est réduit grâce à un abri fiscal.

Facteur-marché boursier $=$ transactions primaires et secondaires des titres de l'entreprise, basées sur l'offre et la demande.

Facteur-marché des métaux $=$ prix des métaux en fonction de l'offre et de la demande, surplus ou pénurie des métaux en fonction de cycles économiques.

Facteur-acteurs

$=\quad$ individus et organisation qui fournissent des biens et services aux investisseurs et entreprises minières et d'exploration.

Facteur-politique

= implication des paliers de gouvernements par le biais de programmes pour encourager l'investissement grâce à la fiscalité.

Facteur-légal

= règles des organismes de régulation du commerce des valeurs mobilières et règles de la Bourse pour pouvoir transiger les titres à sa cote.

Facteur-économique $=$ retombées économiques des dépenses d'exploration et autres.

Facteur-technologique $\quad=$ importance des technologies nouvelles pour 
l'industrie de l'exploration.

Facteur-physique

Facteur-concurrentiel

ENTREPRISE

Ressources humaines

Finances

Technologie

Actifs

Marketing

DECIDEURS

Dirigeants

Conseil d'administration
= inventaire de terrains miniers disponibles à des niveaux différents d'avancement.

= concurrence pour les capitaux et pour les terrains miniers.

$=1 \mathrm{e}$ regroupement de capitaux des ressources humaines et des ressources physiques que forme Normétal inc.

= le niveau d'expertise et les antécédents des ressources humaines dans les sciences de la terre (employés permanents et consultants), dans le domaine financier et du marketing.

= capitaux disponibles pour l'entreprise.

= moyens technologiques utilisés pour la gestion et la production de l'entreprise.

$=$ actifs miniers et financiers de l'entreprise.

= fonction de l'entreprise pour assurer sa promotion.

= individus impliqués dans le processus décisionnel de l'entreprise.

= la direction générale de l'entreprise. = les officiers de l'entreprise. 
RESUME DES CARACTERISTIQUES = DES OPPORTUNITES ET MENACES DE L'ENVIRONNEMENT

énumērer les points négatifs et positifs de chacun des facteurs de l'environnement et décider s'il s'agit d'une menace ou d'une opportunité.

= énumérer les points positifs et négatifs de chacun des indicateurs de l'entreprise et dēcider s'il s'agit d'une force ou d'une faiblesse.

= énumérer ou décrire le système de valeur ET et les aspirations de toutes les personnes impliquées dans le processus décisionnel de 1 'entreprise.

FACTEURS CRUCIAUX DE SUCCES = déterminer lequel ou lesquels des facteurs sont les plus importants pour réussir.

FORCES ET FAIBLESSES = dēterminer les forces et les faiblesses COMPETENCES ET PARTICULARITES relatives de l'entreprise en management DISTINCTES

MISSION, OBJECTIFS PERSONNELS= OBJECTIFS ORGANISATIONNELS marketing, production, finance;

déterminer pourquoi les clients (investisseurs viennent et viendront avec nous plutôt qu'avec une autre compagnie. déterminer la mission de l'entreprise: y a-t-il eu changement ou évolution dans la 
mission?; déterminer si les objectifs personnels et organisationnels sont en conformité avec la mission;

déterminer la concordance de ces éléments avec l'environnement et l'entreprise.

HARMONIE

GUIDE STRATEGIQUE

IDENTIFICATION DES

OPPORTUNITES

EVALUATION DES RISQUES

DECISION
= élimination des écarts trop prononcés.

= dédicer du niveau d'harmonie et du statu quo ou de l'importance de la correction à apporter.

= qantifier et prēciser les opportunitēs à l'intérieur de l'entreprise, à même ses actifs et ses ressources;

quantifier et prēciser les opportunités à 1 'extérieur de l'entreprise pour les actifs et les ressources; préciser une mission différente.

= quantifier les risques de toute nature pour chaque opportunité identifiée.

= choisir et établir la stratégie de l'entreprise. 


\subsection{COMPARAISON AVEC LA REALITE}

Cette démarche consiste à utiliser le modèle conceptuel comme cadre de rēférence pour identifier les changements possibles à la situation décrite dans la première partie.

\subsubsection{Développements}

Depuis le début de l'intervention, certains changements sont intervenus dans 7 'entreprise.

Le portefeuille de propriētēs est passē de deux à six propriētēs. Le nombre d'acres sous le contrôle de l'entreprise est passé de 12 000 à près de 75000 acres.

Deux ententes de coparticipation furent signées sur des projets spécifiques. La première entente le fut avec un tiers et à distance pour l'évaluation d'un résidu minier. La seconde entente fut signée avec une filiale pour l'évaluation de trois propriétés minières.

Les liquidités et les placements se sont accrus de $100 \quad 000 \$$ à 600000 \$. De plus, Normétal inc. est 1'actionnaire majoritaire d'Exploration minière La Sarre inc. Cette activité de création d'une filiale a mobilisé beaucoup d'énergies de la part de la direction.

\subsubsection{Fonds de roulement}

Les développements ont eu pour effet d'amēliorer la situation à court terme. L'entreprise a des liquidités et peu d'obligations. A moyen et long terme, elle fait face aux menaces que l'environnement 
présente, à savoir la disparition du système accréditif.

\subsubsection{Performance boursière}

La performance boursière du titre $n$ 'a pas ètē exceptionnelle (haut $.79 \$$, bas .40 \$). Ceci laisse supposer que les résultats techniques de l'exploration ont été moyens. L'effort de promotion a été limité, car l'entreprise n'a pu tabler sur le niveau d'avancement de ses projets. Les habiletēs du responsable de la promotion n'ont pu supplēer au niveau d'avancement des projets.

\subsubsection{Ressources humaines}

Au niveau des ressources humaines, c'est sensiblement le statu quo. Un peu d'expertise fut apportée par l'arrivée d'un spécialiste des sciences de la terre en géochimie. Une ressource fut ajoutée au service de l'exploration.

\subsubsection{Résultats de l'exploration}

Un succès moyen fut obtenu lors du premier programme d'exploration sur la propriété Barraute. Pour la propriēté Normétal, les attentes étaient fortes; les résultats escomptés ne furent pas obtenus.

\subsubsection{Programe d'acquisition}

Les acquisitions $n$ 'ont pas permis à 1 'entreprise de s'engager dans des projets nouveaux où le niveau d'avancement était près du niveau de production.

Les terrains acquis possèdent un bon potentiel, mais ne sont pas suffisants pour propulser la promotion de l'entreprise et l'assurer de fonds autogénérés. 


\subsubsection{Evolution des projets de 7 'entreprise}

Normétal inc. a procédé lentement lors de l'évaluation de son gîte de zinc. Ce projet peut permettre à l'entreprise d'attirer plus 1 'attention et de générer des revenus.

La négociation et la signature de l'entente de coparticipation pour l'étude de faisabilité du résidu minier furent réalisées avec certaines longueurs. A date, les travaux progressent de façon positive. Ce projet de nature à valoriser l'entreprise n'a pas encore produit tous les effets espérés.

\subsubsection{Mission et objectifs}

La mission de l'entreprise est encore l'exploration. Les objectifs organisationnels semblent prēdominer sur les objectifs personnels, grâce à la création de la filiale. La création d'un groupe semble se dessiner.

\subsection{POINTS FORTS ET FAIBLES}

A la suite de la comparaison du modèle avec la réalité, il s'agit de dégager les principaux points forts ou faibles qui permettent le choix d'une solution à la problématique.

\subsubsection{Situation financière}

Les liquidités peuvent permettre à l'entreprise de saisir une opportunité. Cependant, l'entreprise, si elle saisissait une opportunitē, n'aurait plus de réserves financières devant elle. 


\subsubsection{Propriétés}

Deux propriétés de l'entreprise sont sur le point de devenir des projets de niveaux plus avancés.

\subsubsection{Ressources humaines}

Le manque d'expérience et d'antëcédents des ressources responsables de l'exploration et de la promotion fut un point faible.

\subsubsection{Financement}

L'entreprise a eu et a à sa disposition les fonds nécessaires à ses activités d'exploration. Ces fonds assurent la réalisation des programmes d'exploration planifiés pour une période de 12 mois seulement.

\subsection{RECOMMANDATIONS}

\subsubsection{Stratégie à court terme}

\subsubsection{1 actifs miniers et financiers}

Les actifs actuels constituent une opportunité. L'entreprise devrait concentrer ses efforts sur les projets ou les propriétés les plus avancés. Les autres propriétés devraient faire l'objet d'efforts réduits. Les ressources financières pourraient être plus concentrées et s'avérer plus productives ou tout simplement économisées. Si le projet Normetmar ne peut se concrétiser, les liquidités et la filiale sont des moyens qui doivent être utilisés pour donner accès à l'entreprise à une meilleure ressource physique (terrain minier) que celle déjà en main. 
Le développement du projet Normetmar devrait être accéléré. Si les résultats sont positifs, l'entreprise n'aura pas besoin d'autres propriētés extérieures.

Si le projet de résidu continue à progresser de façon aussi positive et rapide qu'actuellement, il est possible que Normétal inc. doive aller de l'avant avec ce projet en fonction de ses moyens financiers.

L'entreprise doit continuer à mener de front ces deux projets; le premier qui aboutira devra être retenu. Donc, avant de chercher une opportunité à l'extérieur de ses actifs, Normétal inc. doit discriminer parmi les propres opportunités qu'elle possèdé.

Cette recommandation concerne les actifs miniers et financiers, et plus particulièrement, le processus de transformation.

\subsubsection{2 ressources humaines}

L'état du propriétaire-dirigeant pose un problème. En fait, il est difficile d'éliminer un propriētaire majoritaire sans son consentement.

L'expertise manquante devrait plutôt être obtenue par l'utilisation de 1 a consultation, autant pour la fonction marketing que pour la fonction de l'exploration. Au point de vue financier et du marketing, il est reconnu qu'un réseau de contacts élaboré est un atout. L'administration est en voie de construire ce réseau.

L'entreprise devrait mettre sur pied un plan de marketing et procéder à son exécution avec l'aide de consultants. Elle devrait aussi identifier les meilleurs consultants sur le marché pour 
collaborer à l'exploration des terrains en fonction des compétences des consultants et des caractēristiques géologiques des terrains.

Cette recommandation est d'ordre structurel, car elle concerne les tâches et l'utilisation de ressources spécialisées.

\subsubsection{Réêvaluation de la stratégie}

La fonction stratégique est indissociable de toute entreprise. Beaucoup d'entreprises n'arrivent pas à aller plus loin qu'une simple description de ce qu'elles sont. La planification stratégique est d'abord un exercice d'analyse qui permet de situer $l$ 'entreprise dans un environnement complexe et changeant. Une réflexion stratégique permet de questionner à nouveau la mission, le produit, le type de développement, les moyens qu'utilise l'entreprise et les compétences de l'entreprise.

Il serait opportun pour Normétal inc. d'utiliser à nouveau le modèle conceptuel du mémoire de recherche-action pour revoir sa planification stratégique sur une base annuelle ou dès que des changements drastiques sont reconnus. La synchronisation de cette réflexion avec la production du rapport annuel serait pertinente. Ce changement est davantage un changement dans les attitudes et devrait améliorer les attitudes de l'entreprise face à 1 'environnement. 


\section{CONCLUSION}

Dans l'élaboration de la solution, la planification stratégique a été retenue à cause des interrelations des sous-systèmes entre eux, à l'intérieur desquels se retrouve la zone problématique.

Après avoir examiné la littérature sur la planification stratégique, un modèle pour analyser la situation problématique a été présenté.

Ce modèle a été appliqué en comparaison avec la réalité de l'entreprise. Dans le cas de notre entreprise, le questionnement de la réalité décrite à la première partie nous a amené à découvrir une perception différente de la réalité problématique.

Par la suite, nous avons dégagé les principaux points forts et faibles apparus à la suite de la comparaison avec la réalité. Ainsi, nous formulions deux types de recommandations, l'une à court terme et l'autre à moyen terme, en tenant compte des changements possibles, tant au niveau organisationnel que stratégique.

Ainsi, les problèmes d'acquisition des terrains miniers, des résultats de 1 'exploration, de l'école des projets de l'entreprise, des développements, du fonds de roulement, de la performance boursière, de la performance des ressources humaines et de la détermination de la mission de l'entreprise sont considérés en fonction d'une problématique complexe dont les éléments sont interdépendants. 
QUATRIEME PARTIE

CRITIQUE METHODOLOGIQUE 


\section{INTRODUCTION}

Une recherche peut donner lieu à autant de questions que de réponses. C'est pourquoi il est important de comprendre le processus de recherche. Il faut être en mesure de juger du bien-fondé des méthodes utilisées dans la recherche. En fait, il faut savoir si la recherche est valide.

Dans cette dernière partie de la recherche, nous vérifierons si les objectifs de la recherche ont été atteints et comment ils l'ont été. 


\subsection{OBJECTIF DE CREATION D'ENTREPRISE}

Dans la présentation de la recherche, il est indiqué que celle-ci est constituée en partie par une expérience d'entrepreneurship: 1a création d'une entreprise.

\subsubsection{Démarche méthodologique}

Il n'existe pas de méthodologie pour la création d'entreprise. I1 existe cependant des écrits sur le sujet qui prennent la forme de recettes, de conseils, de marches à suivre. Donc, la fiabilité du résultat ne peut se faire que d'une façon: la validation par un tiers.

\subsubsection{Validation par le marché}

Chaque année, de nombreuses entreprises naissent et disparaissent. De toutes tailles, ces entreprises oeuvrent dans toutes les sphëres d'activités.

Ainsi, un travailleur autonome réalise une expérience d'entrepreneurship. La mise sur pied d'un commerce d'alimentation de type "dépanneur" est une création d'entreprise. L'implantation d'une usine de moulin à papier est aussi une crēation d'entreprise.

A partir de quel cycle de vie de l'entreprise, de quelle capitalisation, de quelle quantité de ressources humaines, de quel chiffre d'affaires un entrepreneur a-t-il créē une entreprise? Je pense que beaucoup de réponses sont possibles. De même, la validation peut se faire par de nombreux tiers: fournisseurs, clients, employēs, investisseurs, prêteurs, etc.

A propos de notre expérience d'entrepreneurship, la validation s'est faite par le marché boursier. L'entreprise a du rencontrer de 
nombreuses exigences de la Bourse de Montréal quant à son nombre d'actionnaires, sa capitalisation, ses ressources financières, la qualité de ses projets, la qualité de ses administrateurs et dirigeants, avant de voir l'inscription de son titre à la cote de la Bourse de Montrēal. Cette validation s'est faite après plus d'un an d'opération de l'entreprise et après qu'elle ait réalisée une émission de ses titres auprès du public investisseur. La validation continue de se faire quotidiennement par le marché boursier.

\subsection{OBJECTIF D'AUTODIAGNOSTIC}

Faire de la recherche, c'est chercher à nouveau, examiner quelque chose une seconde fois, plus attentivement, pour en découvrir plusl. L'autodiagnostic est une application pratique de la recherche qui sert à amēliorer la performance de l'entreprise grâce à une meilleure connaissance de celle-ci.

\subsubsection{Problèmes en période de création d'entreprise}

Le choix d'un thème ou sujet de recherche est le résultat d'une prēoccupation en fonction d'un problème. En ce qui nous concerne, les rôles de chercheur et d'acteur ont été influencés par la démarche de création d'entreprise.

Lors de la création d'une entreprise, on touche beaucoup de domaines; il faut atteindre un résultat acceptable. Il faut tout élaborer ou concevoir, soit seul ou avec des collaborateurs. C'est ainsi que l'entrepreneur découvre différentes problématiques et désire maîtriser l'incertitude qu'il découvre. Dans notre cas

1. S.W. Cook, Les méthodes de recherche en sciences sociales, $C$. Selltiz; S. Wrightsmann; Tes éditions HRW, p.2. 
précis, c'est ce qui nous a motivē à analyser la problématique globale décrite dans la présentation de la recherche sous l'angle de la stratégie: la survie de l'entreprise.

\subsubsection{Approche diagnostique}

L'objet d'étude étant une entreprise et le contexte de la recherche favorisant la recherche-action, il fut possible d'utiliser la méthodologie du diagnostic.

La cueillette de données s'est effectuée lors des trois travaux prémémoire. Cette cueillette a été facilitée par la dualité des rôles de chercheur et d'acteur. De plus, l'entreprise était opérationnelle avant le début de 1 a recherche.

Les étapes de la description de la réalité et de la proposition d'un modèle idéalisé furent conservées, mais enrichies de certains éléments provenant de la méthodologie de Peter Chekcland.

L'étape finale du choix des solutions s'est traduite sous la forme de recommandations concrètes, sans implantation. Donc, nous avons agi en expert et, dans ce sens, nous qualifions la méthodologie d' "expert".

\subsubsection{Influence de Chekcland}

Chekcland décrit l'organisation par une image riche pour laquelle il développe les thèmes de super-système, d'environnement, de structure, de processus, de climat organisationnel. Ensuite, lors d'étapes subséquentes, il analyse les problèmes avec un ancrage. L'ancrage correspond à la perspective de l'analyste pour l'étude de la problématique. C'est le point de vue privilégié de l'analyste. Il construit un modèle conceptuel et le compare avec la réalité de 
l'entreprise. Il valide le tout avec les acteurs et procède à l'implantation et réévalue par la suite.

Nous avons retenu les mêmes thèmes que Chekcland, à l'exception du super-système, pour décrire l'entreprise. Cette façon de procēder nous a permis de décrire de façon exhaustive la problématique globale. Elle est globalisante et parfaitement adaptée aux situations complexes. En fait, c'est l'aspect systémique de la méthodologie de Chekcland qui fut retenu.

Le fait d'avoir décrit l'entreprise à la façon de Chekcland nous a fait focaliser sur l'environnement; c'est ainsi que nous avons découvert un environnement complexe. Une autre méthodologie ne nous aurait peut-être pas permis de décrire l'entreprise de façon aussi exhaustive.

L'étape de l'"ancrage", ou plutôt de la perspective pour l'analyse du problème, est prësente. Cependant, l'angle de la stratégie confirme les préoccupations de l'acteur.

L'élaboration du modèle conceptuel (modèle idéal) est le fruit de 1 'expertise du chercheur-acteur et une adaptation des modèles que l'on retrouve dans la littérature des sciences de l'administration.

Pour la comparaison, la dualité des rôles de chercheur-acteur a rendu simultanée l'opération de validation interne.

L'étape d'implantation des recommandations, ou plutôt des solutions, selon la terminologie de Chekcland, s'effectuera dans le temps et l'évaluation pourra se faire dans quelques mois. 


\subsection{OBJECTIF DE REFLEXION CONCEPTUELLE}

L'acquisition de connaissances est un processus continu. Le bon sens nous confine à ce qui nous est familier et plus facilement accessible. Très peu d'écrits structurēs ou plutôt scientifiques existent sur les P.M.E. en exploration. Le bagage de connaissances se trouve confiné à quelques analystes miniers, à quelques journalistes financiers qui véhiculent certains adages ou recettes miracles sur ce type d'organisation. Rappelons seulement le plus flamboyant d'entre les adages: une junior, c'est une affaire de promotion! Est-ce vrai? Ce type d'organisation utilise-t-il des mêmes concepts administratifs que les autres organisations?

\subsubsection{Réflexions sur les concepts administratifs}

\section{typologie}

Dans ce mémoire de recherche-action, une typologie des entreprises minières est présente. Cette typologie précise que le niveau d'avancement des projets est un facteur primordial pour étudier ces entreprises. D'autres facteurs tels le produit, le système clients sont aussi utiles pour l'étude des entreprises d'exploration. Cependant, le niveau d'avancement des projets est indispensable car il situe l'analyste sur la nature même de l'entreprise et sur sa pérennité.

produit

Une réflexion sur la notion de produit est présente dans le travail de recherche. Il apparaît maintenant évident que "même sans échange d'un produit physique avec un client", l'entreprise possède un produit. Ce produit évolue et change avec le temps. L'entreprise doit bien saisir cette évolution pour bien s'adapter. 
client

Le travail de recherche permet d'analyser la manifestation du système client. Nous savons maintenant que l'actionnariat forme le système client. Aussi, le système client évolue ou se modifie pour tenir compte de l'évolution du produit de l'entreprise jusqu'à ce que l'entreprise d'exploration atteigne le stade de la production.

\subsubsection{Connaissances générales}

Dans le mémoire, le lecteur retrouve une description exhaustive du système de financement par actions accrëditives. De plus, certaines données sur l'impact économique du récent développement des entreprises d'exploration sont présentes. Des informations sont aussi présentes sur l'entrepreneurship régional (AbitibiTëmiscamingue). De plus, le lecteur découvre que les connaissances traditionnelles en marketing s'appliquent aux entreprises d'exploration.

\subsection{DUALITE DES ROLES DE CHERCHEUR-ACTEUR}

Des contraintes de temps sont fréquentes dans ce type de rechercheaction, lorsque la recherche est faite sur l'acteur et ses activités et par l'acteur. Dans notre cas, nous avons connu certains délais, surtout à cause de notre rôle d'acteur.

La recherche-action est cependant un moyen efficace de valoriser le rôle de l'Université, de la rapprocher de l'Industrie. Nous sommes alors assurés que la Recherche trouve une application concrète et immédiate dans les entreprises. 


\section{CONCLUSION}

Cette partie du mémoire relate les résultats de la recherche. Chacun des objectifs initiaux de la recherche a été revu et les résultats en regard de ces objectifs ont été énumérēs. Enfin, nous avons indiqué jusqu'à quel point la méthodologie utilisée assurait de la validité des résultats. Une opinion sur l'utilité et les difficultés de la recherche-action a également été émise. 


\section{CONCLUSION GENERALE}

En quoi ce travail constitue-t-il un mémoire de recherche-action pour la maîtrise en P.M.0.? Le programme de maitrise en P.M.O. permet une intervention dans une entreprise existante ou la création d'une entreprise comme base de mémoire. Pour l'intervention dans l'entreprise existante, l'étudiant produit un travail qui prend la forme d'un diagnostic ou d'une analyse quelconque. Pour la création d'entreprise, il existe peu d'antécédents de ce choix. De plus, le programme est muet à ce sujet, du fait qu'il n'existe pas de directive précise sur le travail académique à produire pour évaluer la démarche.

Pour le volet création d'entreprise, l'étudiant pourrait produire un travail de compilation pour rendre compte des actes légaux et administratifs relatifs à la création de l'entreprise. Une simple constatation de l'existence de 1 'entreprise pourrait aussi suffire. De telles prodécures seraient sans doute trop centrées sur 1'action et négligeraient peut-être la recherche si importante au programme de maitrise.

En collaboration avec le responsable du programme, le chercheur-acquis a mis sur pied un démarche qui permette de rendre compte de l'action et de la recherche.

Ce mëmoire innove dans le sens qu'il intègre trois différentes choses dans une production académique. Le chercheur-acteur a voulu dépasser le simple recensement des faits et données. Il a aussi voulu se détacher des styles des études de faisabilité et des études générales. Le mémoire comprend un diagnostic, une réflexion conceptuelle et une évaluation de connaissances. Ce travail permet au chercheur-acteur d'analyser son entreprise en période de mise sur pied. Cette analyse se fait à travers certains concepts et connaissances administratives. Les éléments d'analyse traditionnels (finance, personnel, climat, actifs) sont présents mais ne constituent pas l'essentiel de l'analyse. 
Le chercheur-acteur a voulu approfondir des éléments de connaissance sur le marketing en étudiant les notions de produit et de système client. Une typologie des entreprises d'exploration ainsi que l'explication du processus de transformation d'une entreprise d'exploration renseignent sur la nature des entreprises d'exploration. L'importance du système de financement est mise en perspective. L'analyse de l'entreprise et de ses éléments sert à la mise au point d'une stratëgie d'entreprise.

A la fin de ce travail de recherche, il reste de nombreuses pistes qui méritent une investigation plus approfondie et pourraient constituer des sujets de mémoire en soi. Ces pistes ou domaines d'étude s'appliquent autant à notre entreprise qu'à l'industrie en général.

Comment se fait-il que le processus de transformation ne soit pas plus performant et n'apporte pas plus de découvertes miniēres? Comment les entreprises peuvent-elles contrer la dilution du capital-actions? Comment les entreprises d'exploration peuvent-elles contrôler ou maittriser plus favorablement leur environnement? Quelle est l'importance de l'esprit d'entrepreneurship dans 1 a conduite de projets miniers? Comment se manifeste 1 'entrepreneurship francophone en exploration minière (consultants, contracteurs, compagnies)? Comment les participants à l'industrie de l'exploration perçoivent-ils le risque? Comment la fiscalité affecte un secteur d'activité économique et quelles en sont les retombées sur les entrepreneurs?

Ces interrogations pourraient être le départ d'études descriptives et exploratives pour accumuler de l'information de base sur la problématique globale de l'exploration minière. 


\section{BIBLIOGRAPHIE}

Baril Serge, Etude sur le rēsidu minier de Normétal, Département de technologie minérale du collège de l'Abitibi-Témiscamingue, novembre 1985.

Bērubē Gérard, Initiation aux valeurs mobilières, Publifor, 1984.

Bourse de Montréal, Le financement des Entreprises de ressources, 1985.

Buell Victor, Marketing Management a strategic approach, Mc Graw-Hi11, 1984.

Commission des valeurs mobilières, Le marché des valeurs mobilières, 1985.

Coopers and Lybrand, Financial reporting in Mining Industry, A summary of current and Prefered Practice, 1985.

Dubé Martin, St-Michel André, Etude de prēfaisabilité technique et financière projet Normetmar, 6 mars 1987.

Exploration minière Normétal inc., Rapport annuel 1986, février 1987.

Fiset Gilles, Les compagnies minières junior et 1 a promotion, Département des Sciences économiques et administratives, Université du Québec en AbitibiTémiscamingue, travail de recherche, décembre 1986.

Fiset Gilles, Dan Kobo Ali Lalouali, Projet Normetmar, Département des Sciences économiques et administratives, Université du Québec en AbitibiTémiscamingue, travail de recherche, mars 1987.

Fiset Gilles, Dan Kobo Ali Lalouali, Diagnostic Normétal inc., Département des Sciences économiques et administratives, Université du Québec en AbitibiTémiscamingue, travait de recherche, mars 1987.

Franzen, J.P., "The Juniors - A good bet for an Investing Strategy." The Northern Miner, vol. 72 , no. 14.

Godet Michel, Prospective et planification stratégique, Editions Economica, 1985.

Gelltiz, C., Wrightsman, S., Cook, S.W., Les méthodes de recherche en sciences sociales, traduit par D. Bêlanger, Les Editions HBW, 1977. 
Hunt Peter, "Confidence and Trust are the essence of good public relation", The Northern Miner, Vol. 71, no. 15.

Martinet Alain Charles, Management stratëgique: organisation et politique, Mc Graw-Hi11, 1984.

Ministère de l'Energie et des Ressources, Secteur mines, Répertoire des établissements menant des opérations minières au Québec en 1984, Gouvernement du Québec, 1985.

Prévost Paul, Le Diagnostic Intervention: une approche systémique au diagnostic organisationnel et à la recherche action, Departement des Sciences économiques et administratives, Université du Québec à Chicoutimi, 1983.

Samson Bēlair, Exploration minière Normétal inc., Rapport financier initial, 6 décembre 1985, décembre 1985.

Sociētē Conseil Maheu Noiseux et Roche 1 tēe, Etude d'impact économique sur le financement des dépenses d'exploration miniēre au moyen d'actions accréditives et de sociétés en commandite, document révisé le 26 janvier 1987 .

Sociétē en commandite La Sarre et Sociēté d'Exploration minière La Sarre inc., Prospectus, 17 juin 1987.

Thiétart Raymond Alain, La stratégie d'entreprise, Mc Graw-Hill, 1984. 
MEMBRES DE LA BOURSE DE MONTREAL

ALLENVEST GROUP LIMITED

SOCIETE DE COURTAGE BACHE INC.

BELL GDUINLDCK LIMITED

BRAULT, GUY, O'BRIEN INC.

L.A. BRENZEL SECURITIES LIMITED

BROLWN, BALDWIN, NISKER LIMITED

BUNTING \& CO. LIMITED, ALFRED

BURNS FRY LIMITED

CANARIM INVESTMENT CORPORATION LTD.

CANAVEST HOUSE LIMITED

CASGRAIN \& COMPAGNIE LIMITEE

CHISHOLM \& CO. LIMITED, HECTOR M.

CONTINENTAL CARLISLE DOUGLAS

DAVIDSON PARTNERS LIMITED

DEACON, HODGSON INC. F.H.

DEAN WITTER REYNDLDS (CANADA) INC.

DE LEEUW \& ASSOCIES, CDURTIER EN VALEURS MOBILIERES INC.

DERAGON, LANGLOIS LIMITEE

DOMINICK CORPORATION OF CANADA LIMITED

DOMINION SECURITIES PITFIELD LIMITED

FIRST CANADA SECURITIES INTL. LTD.

FIRST MARATHON SECURITIES LIMITED

GARDINER GRDUP STOCKBROKERS INC.

GARDINER, WATSON LIMITED

GEDFFRION, LECLERC INC.
GDRDON CAPITAL CORPQRATION

GREAT PACIFIC MANAGEMENT CO. LTD. LES INUESTISSEMENTS DISNAT INC. GRAHAM \& COMPANY LIMITED, JOHN LAFERTY, HARWDOD \& PARTNERS LTD. LATIMER CO. LIMITED, W.D.

LEDUC \& ASSOCIES VALEURS MOBILIERES INC.

LEVESQUE, BEAIJBIEN INC.

LOEWEN, DNDAATJE, ME CUTCHEON \& CO. LTD.

Mac dougall, Mac dougall, Mac tief INC.

MACK LIMITED, J.D.

MAISON PLACEMENT CANADA INC.

MC DERMID ST. LAWRENCE LIMITED

ME LEAN ME CARTY LIMITED

ME LEOD YOUNG WEIR LIMITEE

ME NEIL, MANTHA INC.

MERIT INVESTMENT CORPORATION

MERRILL LYNCH CANADA INC.

MIDLAND DOHERTY LIMITEE

MOLSON, RDUSSEAU INC.

NESBITT, THOMSON, BONGARD INC.

OLIVER \& COMPANY LIMITED, C.M.

OSLER, WILLS, BICKLE LIMITED

PEMBERTON HOUSTON WILLOUGHBY INC. 
POLLITT, LEGAULT \& CO. INC.

PDPE, JDSEPH (PDPE \& CD.)

RABIN, BUDDEN, PARTERS

RASMUSSEN, SHARP \& COMPANY LTD.

RESEARCH SECURITIES OF CANADA LTD.

RICHARDSON GREENSHIELDS OF CANADA

LIMITED

ROD J. HAMILTON \& ASSOCIES INC.

FOYAL DAK SECURITIES CORP.

SCOTIA BDND COMPANY LIMITED

TASSE \& ASSOCIES LIMITEE

TAYLOR \& COMPANY LIMITEE, D.W.

WALWYN STODGELLL COCHRAY MURRAY LTEE

WODD GUNDY LTEE

YORKTON SECURITIES INC. 


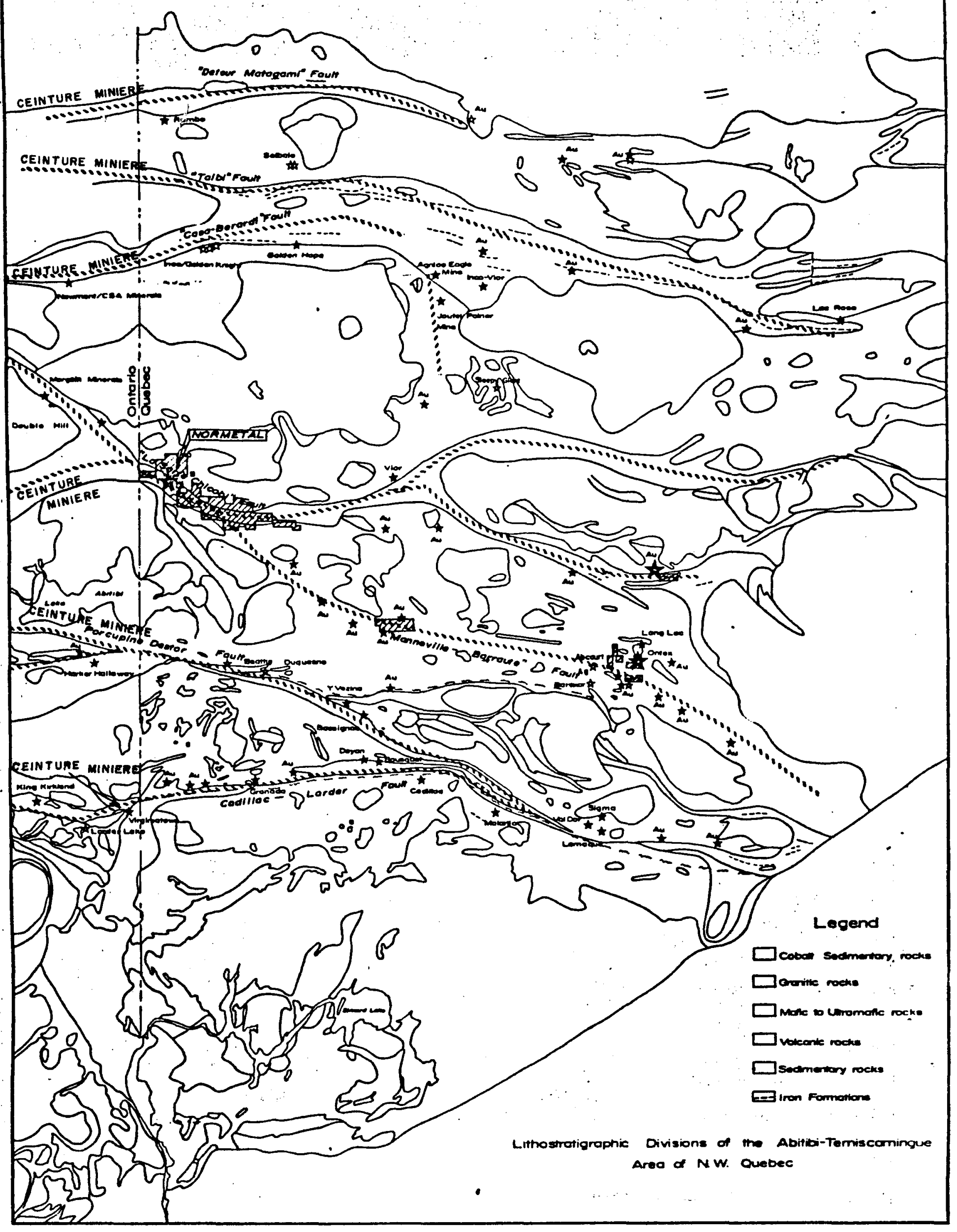

